\title{
All fundamental electrically charged thin shells in general relativity: From star shells to tension shell black holes and regular black holes and beyond
}

\author{
José P. S. Lemos* \\ Centro de Astrofísica e Gravitação - CENTRA, Departamento de Física, Instituto Superior Técnico - IST, \\ Universidade de Lisboa - UL, Av. Rovisco Pais 1, 1049-001 Lisboa, Portugal \\ Paulo Luz \\ Centro de Astrofísica e Gravitação - CENTRA, Departamento de Física, \\ Instituto Superior Técnico - IST, Universidade de Lisboa - UL, \\ Av. Rovisco Pais 1, 1049-001 Lisboa, Portugal, \\ Center for Mathematical Analysis, Geometry and Dynamical Systems, \\ Instituto Superior Técnico - IST, Universidade de Lisboa - UL, \\ Avenida Rovisco Pais 1, 1049 Lisboa, Portugal, and \\ Departamento de Matemática, ISCTE - Instituto Universitário de Lisboa, Portugal
}

\begin{abstract}
We classify all fundamental electrically charged thin shells in general relativity, i.e., static spherically symmetric perfect fluid thin shells with a Minkowski spacetime interior and a ReissnerNordström spacetime exterior, characterized by the spacetime mass $M$, which we assume positive, and the electric charge $Q$, which without loss of generality in our analysis can always be assumed as being the modulus of the electric charge, be it positive or negative. The fundamental shell can exist in three states, namely, nonextremal when $\frac{Q}{M}<1$, which includes the Schwarzschild $\frac{Q}{M}=0$ state, extremal when $\frac{Q}{M}=1$, and overcharged when $\frac{Q}{M}>1$. The nonextremal state, $\frac{Q}{M}<1$, allows the shell to be located in such a way that the radius $R$ of the shell can be outside its own gravitational radius $r_{+}$, i.e., $R>r_{+}$, where $r_{+}$is given in terms of $M$ and $Q$ by $r_{+}=M+\sqrt{M^{2}-Q^{2}}$, or can be inside its own Cauchy radius $r_{-}$, i.e., $R<r_{-}$, where $r_{-}$is given in terms of $M$ and $Q$ by $r_{-}=M-\sqrt{M^{2}-Q^{2}}$. The extremal state, $\frac{Q}{M}=1$, allows the shell to be located in such a way that the radius $R$ of the shell can be outside its own gravitational radius $r_{+}$, i.e., $R>r_{+}$, where now $r_{+}=r_{-}$, or can be inside its own gravitational radius, i.e., $R<r_{+}$, or can be at its own gravitational radius $r_{+}$, i.e., $R=r_{+}$. The overcharged state, $\frac{Q}{M}>1$, allows the shell to be located anywhere $R \geq 0$. There is yet a further division, indeed, one has still to specify the orientation of the shell, i.e., whether the normal out of the shell points toward increasing radii or toward decreasing radii. For the shell's orientation, the analysis in the nonextremal state is readily performed using Kruskal-Szekeres coordinates, whereas in the extremal and overcharged states the analysis can be performed in the usual spherical coordinates. There is still a subdivision in the extremal state $r_{+}=r_{-}$when the shell is at $r_{+}, R=r_{+}$, in that the shell can approach $r_{+}$from above or approach $r_{+}$from below. The shell is assumed to be composed of an electrically charged perfect fluid characterized by the energy density, pressure, and electric charge density, for which an analysis of the energy conditions, null, weak, dominant, and strong, is performed. In addition, the shell spacetime has a corresponding Carter-Penrose diagram that can be built out of the diagrams for Minkowski and Reissner-Nordström spacetimes. Combining these two characterizations, specifically, the physical properties and the Carter-Penrose diagrams, one finds that there are fourteen cases that comprise a bewildering variety of shell spacetimes, namely, nonextremal star shells, nonextremal tension shell black holes, nonextremal tension shell regular and nonregular black holes, nonextremal compact shell naked singularities, Majumdar-Papapetrou star shells, extremal tension shell singularities, extremal tension shell regular and nonregular black holes, Majumdar-Papapetrou compact shell naked singularities, Majumdar-Papapetrou shell quasiblack holes, extremal null shell quasinonblack holes, extremal null shell singularities, Majumdar-Papapetrou null shell singularities, overcharged star shells, and overcharged compact shell naked singularities.
\end{abstract}

\footnotetext{
* joselemos@ist.utl.pt

† paulo.luz@ist.utl.pt
} 


\section{INTRODUCTION}

An important group of solutions in a theory of gravitation, in particular solutions to general relativity, are those that represent stars. A nonrotating star in general relativity is a static spherically symmetric solution composed of some matter fields that either extend indefinitely, with its fields decaying sufficiently fast to yield a well-defined asymptotic infinity structure, or fill some interior part that has a well defined boundary that in turn connects to a vacuum exterior. A particular interesting example of this latter instance of a general relativistic star is an interior composed of vacuum plus a boundary made of a thin shell of matter, that is joined to a vacuum exterior. The matter concentration on the thin shell can produce with faithfulness several local and global properties of the spacetime, with particular relevance it can generate the formation of apparent and event horizons.

In general relativity the generic study of uncharged timelike and spacelike thin shells was initiated by Israel [1], followed by Papapetrou and Hamoui [2] and Taub [3], with lightlike thin shells being treated by Barrabès and Israel [4]. In the instance that the uncharged thin shell is spherically symmetric, the interior spacetime can be of any type, in many situations Minkowski can be used, and the exterior spacetime of pure vacuum is the Schwarzschild spacetime according to Birkoff's theorem. There are many applications of spherically symmetric uncharged shells with a Schwarzschild exterior and we mention a few of those. Thin shell gravitational collapse was treated by Israel [5], spacetimes with counter-rotating particles in thin shells were studied by Evans [6] and Papapetrou and Hamoui [7], collisions of spherical thin shells were analyzed by 't Hooft and Dray [8], cosmic bubbles with inflation were considered by Blau, Guendelman, and Guth [9], properties of thin shells with a black hole interior were investigated by Frauendiener, Hoenselaers, and Konrad [10] and Brady, Louko and Poisson [11], the study of tension shell black holes was performed by Katz and Lynden-Bell [12] and Comer and Katz [13], thin shell wormholes were constructed by Visser [14] and by Lemos, Lobo, and Oliveira [15], and properties of black holes, such as black hole entropy through thin shells, were done by André, Lemos, and Quinta [16] and Bergliaffa, Chiapparini, and Reyes [17].

The study of spherically symmetric electrically charged thin shells with a Reissner-Nordström spacetime exterior was dealt by de la Cruz and Israel [18] and Kuchař [19], gravitational collapse of electrically charged shells was performed by Chase [20] and Boulware [21], Vilenkin and Fomin [22] inspected the problem of the self-energy of the electron through thin shells, the topology of the collapse of charged thin shells and fluids was analyzed by Hiscock [23], interacting electric thin shells were studied in Lemos and Zanchin, [24], Dias, Gao, and Lemos studied electric collapsing shells in Lovelock theory [25] with the general relativistic counterpart and its relation to cosmic censorship studied by Gao and Lemos [26], properties of highly compact electric shells together with their energy conditions where a maximum bound, of the kind of the Buchdahl bound has been found, have been discussed by Andréasson [27], the inclusion of a Vlasov fluid into a thin shell was studied by Andréasson, Eklund, and Rein [28], thin shell electrically charged wormholes were constructed by Dias and Lemos [29], regular black holes with thin shells were worked out in [30], the highest compact shells were analyzed by Lemos and Zaslavskii [31], Berezin and Dokuchaev performed a thorough study of gravitational collapse of electric thin shells [32], and properties of black holes, such as black hole entropy, through thin shells, were analyzed by Lemos, Quinta, and Zaslavskii [33, 34], tension electric shells on the other side of the Reissner-Nordstöm universe were studied by Luz and Lemos [35], further study on thin shells with matter obeying the Einstein-Maxwell equations with the inclusion of a Vlasov fluid were analyzed in [36], and for the highest compact electric thin shells that form quasiblack holess see the work of Lemos and Zaslavskii [37].

A feature of the electric thin shells is that the exterior Reissner-Nordström spacetime can be in three different states depending on the ratio of the charge $Q$ to spacetime mass $M, \frac{Q}{M}$, noting that we use units in which the constant of gravitation and the speed of light are equal to one. Indeed, the exterior Reissner-Nordström spacetime can be nonextremal when $\frac{Q}{M}<1$, extremal when $\frac{Q}{M}=1$, and overcharged when $\frac{Q}{M}>1$. The matter that forms the thin shells can be of several types, one type often considered is an electric charged perfect fluid, with energy density $\sigma$ and pressure $p$. Of course the electric charge density $\sigma_{e}$ of the fluid is related to $Q$ and the rest mass density of the fluid $\sigma$ is related to $M$. An interesting particular situation is when $\sigma_{e}=\sigma$, for which the matter is called Majumdar-Papapetrou matter and which gives rise to an exterior extremal Reissner-Nordström spacetime with $\frac{Q}{M}=1$. For all the three different states it is of interest to test the energy conditions for the matter, the most important of which are the null, weak, dominant and strong. Many of the studied shell spacetimes can only be understood through the maximal extension of the corresponding exterior spacetime. Indeed, to appreciate a spacetime in its totality, in particular a spherical symmetric spacetime, one should maximally extend it and draw the corresponding Carter-Penrose diagram by using the techniques available, see, e.g., [38-43]. In addition, to have a full grasp on electric shells, certain properties of the Reissner-Nordström spacetime have to be understood, such as the regions where electric repulsion dominates over gravitational attraction.

The aim of the paper is to find and classify all the fundamental electrically charged thin shells in the EinsteinMaxwell theory, i.e., static spherically symmetric electrically charged general relativistic thin shells with a Minkowski interior and a Reissner-Nordström exterior. We thus extend the Katz-Lynden-Bell solution and analysis done for uncharged shells with a Schwarzschild exterior. The fundamental electric thin shells are assumed to have a perfect 
fluid stress-energy tensor $S_{\alpha \beta}$ on the shell which is defined through the junction of the interior and exterior spacetimes. We consider that the shells have positive spacetime mass $M$, in the no-shell limit they have zero mass, $M \geq 0$, and the radius of the shell obeys $R \geq 0$. In the nonextremal state, $\frac{Q}{M}<1$, there are two natural intrinsic radii, the gravitational radius $r_{+}$given by $r_{+}=M+\sqrt{M^{2}-Q^{2}}$ and the Cauchy radius $r_{-}$given by $r_{-}=M-\sqrt{M^{2}-Q^{2}}$. Depending on the location radius $R$ of the shell, $r_{+}$and $r_{-}$can be horizon radii. In this nonextremal state it is useful to define Kruskal-Szekeres coordinates for the maximally extended spacetime, as they allow to analyze in a natural way the physical properties including the energy conditions of the thin shell at any allowable location radius $R$. In the extremal state, $\frac{Q}{M}=1$, the two intrinsic radius merge into one, $r_{+}=r_{-}$, and it is possible to analyze the physical properties of the thin shell including the energy conditions at any allowable location radius $R$ simply by resorting to the usual spacetime spherical coordinates. In the overcharged state, $\frac{Q}{M}>1$, the two intrinsic radius do not exist, one can analyze the physical properties of the thin shell including the energy conditions at any allowable location radius $R$ also by resorting to the usual spacetime spherical coordinates. The shell spacetime classification that we present can only be fully understood through the maximal extension of the outer Reissner-Nordström spacetime and the drawing of the Carter-Penrose diagrams. A bewildering variety of fourteen cases appear, namely, nonextremal star shells, nonextremal tension shell black holes, nonextremal tension shell regular and nonregular black holes, nonextremal compact shell naked singularities, Majumdar-Papapetrou star shells, extremal tension shell singularities, extremal tension shell regular and nonregular black holes, Majumdar-Papapetrou compact shell naked singularities, Majumdar-Papapetrou shell quasiblack holes, extremal null shell quasinonblack holes, extremal null shell singularities, Majumdar-Papapetrou null shell singularities, overcharged star shells, and overcharged compact shell naked singularities. In contrast to the Schwarzschild shell analyzed by Lynden-Bell and Katz which has only two cases, here we have indeed a wealth of cases. To all the fourteen cases, a physical interpretation can be given with the help of the two main features that we mentioned, namely, the matter properties in conjunction with the energy conditions, and the causal and global structure based on the Carter-Penrose diagrams. Which cases are familiar and which cases are peculiar, or even strange, depends on the analysis one makes. Some cases have the energy conditions verified and the geometrical setup seems to be physically reasonable, other cases have the energy conditions verified and the resulting spacetime is rather peculiar, and yet other cases have the energy conditions violated with a physically reasonable geometrical setup.

The article is organized as follows. In Section II, we set the framework and devise the manner to study the physical properties of the fundamental electric thin shells through the formalism of junction conditions in general relativity, joining a Minkowski interior to a Reissner-Nordström exterior. We present the main features of the Minkowski and the Reissner-Nordström spacetimes together with their Carter-Penrose diagrams. We also establish the classification scheme and the nomenclature we use. In Section III, we study nonextremal electric thin shells outside the gravitational radius and show there are two types, namely, star shells and tension shell black holes. In Section IV, we study nonextremal electric thin shells inside the Cauchy radius and show there are two types, namely, tension shell regular and nonregular black holes and compact shell naked singularities. In Section V, we study extremal electric thin shells outside the gravitational radius and show there are two types, namely, Majumdar-Papapetrou star shells and tension shell black holes. In Section VI, we study extremal electric thin shells inside the gravitational radius and show there are two types, namely, tension shell regular and nonregular black holes and Majumdar-Papapetrou compact shell naked singularities. In Section VII, we study extremal electric thin shells at the gravitational radius and show there are four types, namely, Majumdar-Papapetrou shell quasiblack holes, extremal null shell quasinonblack holes, extremal null shell singularities, and Majumdar-Papapetrou null shell singularities. In Section VIII, we study overcharged thin shells and show there are two types, namely, star shells and compact shell naked singularities. In Section IX, we study the weak, null, dominant, and strong energy conditions for all the fundamental electric thin shells and present a chart with all Carter-Penrose diagrams for the shells. In Section X, we conclude. In Appendix A, we present the maximal extension of the nonextremal Reissner-Nordström spacetime through Kruskal-Szekeres coordinates important to deal with the shells in a nonextremal state. In Appendix B, we present the calculation of the shell's extrinsic curvature in a nonextremal Reissner-Nordström exterior spacetime also important to the whole development of the paper. 


\section{PRELIMINARIES: PHYSICAL PROPERTIES OF FUNDAMENTAL ELECTRIC THIN SHELLS THROUGH JUNCTION CONDITIONS, MINKOWSKI INTERIOR AND REISSNER-NORDSTRÖM EXTERIOR AND THEIR CARTER-PENROSE DIAGRAMS, AND CLASSIFICATION SCHEME AND NOMENCLATURE}

\section{A. Physical properties of fundamental electric thin shells through junction conditions}

We work with general relativity coupled to electric matter, so the appropriate equations are the Einstein-Maxwellcharged matter field equations, i.e.,

$$
\begin{aligned}
R_{\alpha \beta}-\frac{1}{2} g_{\alpha \beta} R & =8 \pi T_{\alpha \beta}, \\
\nabla_{\beta} F^{\alpha \beta} & =4 \pi J^{\alpha},
\end{aligned}
$$

where $R_{\alpha \beta}$ is the Ricci tensor, $R$ the Ricci scalar, $T_{\alpha \beta}$ the stress-energy tensor, $F_{\alpha \beta}$ the Faraday-Maxwell tensor, $J^{\alpha}$ is the electromagnetic 4-current, and $\alpha, \beta=0,1,2,3$ are the usual spacetime indices. The other Maxwell equations, $\nabla_{[\alpha} F_{\beta \gamma]}=0$, where square brackets represent antisymmetrization in the delimited indices, are automatically satisfied for a properly defined $F_{\alpha \beta}$. We use units in which the constant of gravitation and the speed of light are equal to one, and assume the metric signature $(-+++)$.

We consider a general relativistic spacetime that is built from an interior $\mathcal{M}_{\mathrm{i}}$ with metric $g_{\mathrm{i}}$, an exterior $\mathcal{M}_{\mathrm{e}}$ with metric $g_{\mathrm{e}}$, glued together at a common hypersurface $\mathcal{S}$. We will assume $\mathcal{M}_{\mathrm{i}}$ to be described by the Minkowski solution and $\mathcal{M}_{\mathrm{e}}$ to be the Reissner-Nordström spacetime, but for the time being we can keep the analysis quite general. In joining $\mathcal{M}_{\mathrm{i}}$ with $\mathcal{M}_{\mathrm{e}}$ the whole spacetime solution $\mathcal{M}$ has still to obey the Einstein-Maxwell field equations, Eqs. (1) and (2). The hypersurface $\mathcal{S}$ can be timelike, lightlike, or spacelike. Here, we work with the timelike and possible spacelike situations, and we will revise briefly the Darmois-Israel junction formalism for these type of hypersurfaces in the theory of general relativity [1], see also [19] for the inclusion of the electromagnetic field. The lightlike case can sometimes be dealt with by extension of these two situations or generically within an appropriate formalism [4].

To start, we assume that it is possible to define a common coordinate system $\left\{x^{\alpha}\right\}$ on both sides of the hypersurface $\mathcal{S}$, where the index $\alpha$ runs from 0 to 3, for the time and the three space components, respectively. We also assume the existence of a vector field $n$, well defined on both sides of $\mathcal{S}$, to be orthogonal at each point to the matching surface. We choose $n$, the normal to $\mathcal{S}$, to point from $\mathcal{M}_{\mathrm{i}}$ to $\mathcal{M}_{\mathrm{e}}$ and without loss of generality

$$
n^{\alpha} n_{\alpha}=\varepsilon
$$

where $n^{\alpha}$ are the components of $n$ in the coordinate system $\left\{x^{\alpha}\right\}$ and $\varepsilon$ is \pm 1 depending on $n$ being spacelike or timelike, respectively, the null case having $\varepsilon=0$ but will not be treated here. The normal vector field $n$ is such that it is spacelike or timelike if the hypersurface is timelike or spacelike, respectively. We denote by $\left\{y^{a}\right\}$ a local coordinate system on $\mathcal{S}$, where the index a has three components only, which depending on the character of $\mathcal{S}$, can be one for the time and two for the other space coordinates, or three for the space coordinates. Now, the normal vector field $n$ must be orthogonal, at each point, to the tangent vectors to the hypersurface $\mathcal{S}, e_{a} \equiv \frac{\partial}{\partial y^{a}}$, such that

$$
e_{a}^{\alpha} n_{\alpha}=0
$$

with $e_{a}^{\alpha} \equiv \frac{\partial x^{\alpha}}{\partial y^{a}}$. The induced metric on $\mathcal{S}$ as seen from each region $\mathcal{M}_{\mathrm{i}}$ and $\mathcal{M}_{\mathrm{e}}$, is

$$
h_{\mathrm{i} a b} \equiv g_{\mathrm{i} \alpha \beta} e_{a}^{\alpha} e_{b}^{\beta}, \quad h_{\mathrm{e} a b} \equiv g_{\mathrm{e} \alpha \beta} e_{a}^{\alpha} e_{b}^{\beta}
$$

respectively, where $g_{\mathrm{i} \alpha \beta}$ and $g_{\mathrm{e} \alpha \beta}$ are the components of the interior and exterior metrics in the coordinate system $\left\{x^{\alpha}\right\}$, respectively. Notice that in general, the induced metric on $\mathcal{S}$ by each metric $g_{\mathrm{i} \alpha \beta}$ or $g_{\mathrm{e} \alpha \beta}$ may not coincide, hence we use the notation $h_{\mathrm{i} \alpha \beta}$ and $h_{\mathrm{e} \alpha \beta}$ to refer to the metric induced by each spacetime $\mathcal{M}_{\mathrm{i}}$ and $\mathcal{M}_{\mathrm{e}}$, respectively. The extrinsic curvature $K_{\mathrm{i} a b}$ or $K_{\mathrm{e} a b}$ of $\mathcal{S}$, as an embedded manifold in $\mathcal{M}_{\mathrm{i}}$ or $\mathcal{M}_{\mathrm{e}}$, respectively, is defined as

$$
K_{\mathrm{i} a b} \equiv e_{a}^{\alpha} e_{b}^{\beta} \nabla_{\mathrm{i} \alpha} n_{\beta}, \quad K_{\mathrm{e} a b} \equiv e_{a}^{\alpha} e_{b}^{\beta} \nabla_{\mathrm{e} \alpha} n_{\beta}
$$

where $\nabla_{\mathrm{i}}$ and $\nabla_{\mathrm{e}}$ are the covariant derivatives with respect to $g_{\mathrm{i} \alpha \beta}$ and $g_{\mathrm{e} \alpha \beta}$, respectively. Their traces are

$$
K_{\mathrm{i}} \equiv h_{\mathrm{i}}^{a b} K_{\mathrm{i} a b}, \quad K_{\mathrm{e}} \equiv h_{\mathrm{e}}^{a b} K_{\mathrm{e} a b}
$$

respectively. 
We need to find the conditions under which the matching of the two spacetimes $\mathcal{M}_{\mathrm{i}}$ and $\mathcal{M}_{\mathrm{e}}$ form a valid solution of the field equations, namely, of Eqs. (1) and (2). So, following the Darmois-Israel formalism [1] to join the two regions $\mathcal{M}_{\mathrm{i}}$ and $\mathcal{M}_{\mathrm{e}}$ at $\mathcal{S}$, such that the union of $g_{\mathrm{i} \alpha \beta}$ and $g_{\mathrm{e} \alpha \beta}$ forms a valid solution to the Einstein field equations (1), two junction conditions must be verified at the matching surface $\mathcal{S}$ : (i) The induced metric as seen from each region $\mathcal{M}_{\mathrm{i}}$ and $\mathcal{M}_{\mathrm{e}}$, see Eq. (5), must be the same, i.e.,

$$
\left[h_{a b}\right]=0
$$

where, we use $[\psi]$ to represent the difference of a field as seen from each sub-manifold at $\mathcal{S}$, i.e., $\left.[\psi] \equiv \psi_{\mathrm{e}}\right|_{\mathcal{S}}-\left.\psi_{\mathrm{i}}\right|_{\mathcal{S}}$, with $\psi_{\mathrm{i}}$ and $\psi_{\mathrm{e}}$ referring to a field $\psi$ defined in $\mathcal{M}_{\mathrm{i}}$ or $\mathcal{M}_{\mathrm{e}}$, respectively; (ii) If the extrinsic curvature, see Eq. (6), is not the same on both sides of the boundary $\mathcal{S}$, then a thin shell is present at $\mathcal{S}$. The relation between the extrinsic curvature $K_{a b}$ of $\mathcal{S}$ and the stress-energy tensor $S_{a b}$ of the thin shell is given by

$$
-\varepsilon\left(\left[K_{a b}\right]-h_{a b}[K]\right)=8 \pi S_{a b}
$$

We further assume that the stress-energy $S_{a b}$ of the thin shell is a perfect fluid stress-energy tensor on $\mathcal{S}$, i.e., we assume that the total stress-energy tensor, defined as the sum of the matter stress-energy tensor and the electromagnetic stress-energy tensor, can be written as

$$
S_{a b}=\sigma u_{a} u_{b}+p\left(h_{a b}+u_{a} u_{b}\right)
$$

where $\sigma$ is the energy density, $p$ is the pressure, and $u_{a}$ is the fluid's velocity on $\mathcal{S}$. This $u_{a}$ is uniquely defined from projecting the interior four-velocity $u_{\mathrm{i}}{ }^{\alpha}$ onto $\mathcal{S}$ itself as $u_{a}=u_{\mathrm{i}}{ }^{\alpha} e_{a}^{\alpha}$, or projecting the exterior four-velocity $u_{\mathrm{e}}{ }^{\alpha}$ onto $\mathcal{S}$ itself as $u_{a}=u_{\mathrm{e}}{ }^{\alpha} e_{a}^{\alpha}$, with both projections obviously yielding the same $u_{a}$.

In the presence of electromagnetic fields, to guarantee that the spacetime $\mathcal{M}$ is a valid solution of the Maxwell field equations, Eq. (2), in addition to Eqs. (8) and (9) we must impose that the Faraday-Maxwell tensor, $F_{\alpha \beta}$, obeys certain conditions. Defining the projected Faraday-Maxwell tensor at $\mathcal{S}$ from the interior as as $F_{\mathrm{i} a b}=F_{\mathrm{i} \alpha \beta} e_{a}^{\alpha} e_{b}^{\beta}$ and from the exterior as $F_{\mathrm{e} a b}=F_{\mathrm{e} \alpha \beta} e_{a}^{\alpha} e_{b}^{\beta}$, and the projected Faraday-Maxwell vector at $\mathcal{S}$ from the interior as as $F_{\mathrm{i} a}=F_{\mathrm{i} \alpha \beta} e_{a}^{\alpha} n^{\beta}$, and from the exterior as $F_{\mathrm{e} a}=F_{\mathrm{e} \alpha \beta} e_{a}^{\alpha} n^{\beta}$, the electromagnetic matching conditions at $\mathcal{S}$ are then

$$
\left[F_{a b}\right]=0
$$

and

$$
\left[F_{a}\right]=4 \pi s_{a}
$$

where $s_{a}$ is the electromagnetic surface current at $\mathcal{S}$ given by

$$
s_{a}=\sigma_{e} u_{a}
$$

with $\sigma_{e}$ being the electric charge density on $\mathcal{S}$.

Two notes are in order. The first note is to mention that an infinitesimally thin shell is certainly an approximation to a thick shell with very small thickness. The second note is to draw the attention that our assumption on the form of $S_{a b}$, see Eq. (10), is a restriction on the properties of the fluid, namely, it imposes that the thin shell fluid has no effective anisotropic pressure. Since under certain density regimes, realistic matter is expected to be anisotropic, it would be interesting to understand how the presence of anisotropic pressure affects our results. Surely, both assumptions, namely, infinitesimal thickness and isotropic matter for the shell, simplify the analysis, which nevertheless, as we will see, can be quite complex.

\section{B. Minkowski interior and Reissner-Nordström exterior spacetimes}

\section{Minkowski interior spacetime}

The interior spacetime $\mathcal{M}_{\mathrm{i}}$ that will be considered is the Minkowski spacetime. The Minkowski spacetime is a totally empty spacetime and is a solution, a trivial solution, to the Einstein-Maxwell equations, Eqs. (1) and (2). It is characterized in spherical coordinates by the following line element

$$
d s^{2}=-d t^{2}+d r^{2}+r^{2} d \Omega^{2},
$$


where $t$ and $r$ are the time and radial coordinates, respectively, and $d \Omega^{2} \equiv d \theta^{2}+\sin ^{2} \theta d \varphi^{2}$, with $\theta$ and $\varphi$ being the angular coordinates.

The Carter-Penrose diagram of the Minkowski is given in Figure 1. It is worth noting the timelike line $r=0$ which is the origin of coordinates, the null infinities, the past one $\mathscr{I}^{-}$and the future one $\mathscr{I}^{+}$, and the spacelike infinity $i_{0}$. There are also the timelike infinities $i^{-}$and $i^{+}$at the lower and upper vertices of the triangle that are not drawn to not overload the figure. Each point in the diagram represents a two-sphere of radius $r$.

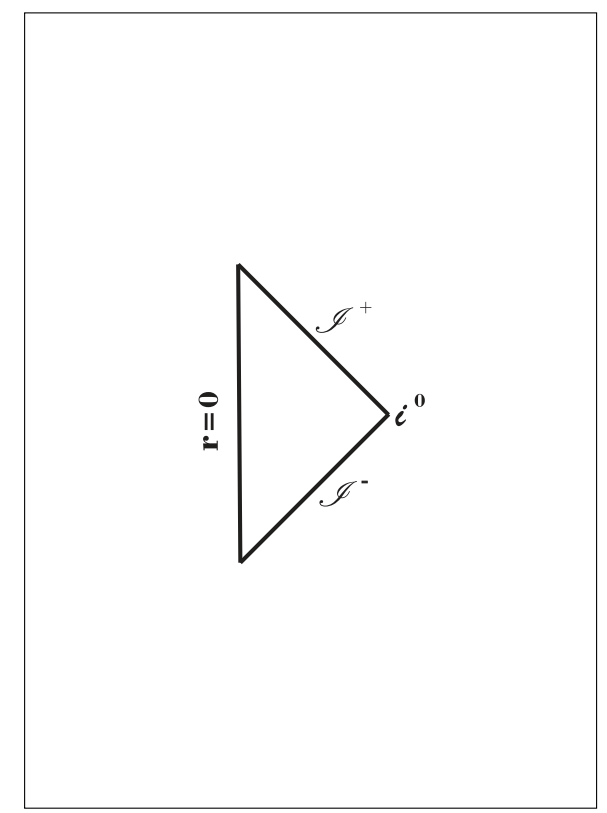

Figure 1: Carter-Penrose diagram of a spacetime described by the Minkowski solution. See text for details.

\section{Reissner-Nordström exterior spacetime}

The exterior spacetime $\mathcal{M}_{\mathrm{e}}$ that will be considered is the Reissner-Nordström spacetime. The Reissner-Nordström spacetime is a solution fo the Einstein-Maxwell equations, Eqs. (1) and (2), and describes an empty massive electrically charged spherically symmetric spacetime. The full vacuum Reissner-Nordström spacetime is the unique vacuum spherically symmetric electric charged solution in general relativity, following Birkhoff's theorem. In what are commonly called Schwarzschild coordinates $(t, r, \theta, \varphi)$, the Reissner-Nordström spacetime is a solution of the Einstein-Maxwell field equations characterized by the following line element

$$
d s^{2}=-\left(1-\frac{2 M}{r}+\frac{Q^{2}}{r^{2}}\right) d t^{2}+\frac{d r^{2}}{1-\frac{2 M}{r}+\frac{Q^{2}}{r^{2}}}+r^{2} d \Omega^{2} .
$$

with $M$ being the spacetime mass and $Q$ its charge. We assume the mass to be zero or positive $M \geq 0$ and $Q$ we treat, without loss of generality here, as the modulus of the electric charge, be it positive or negative. A function that turns up often is the redshift function $k(r, M, Q)$ at some radius $r$, or simply $k$, given by

$$
k=\sqrt{1-\frac{2 M}{r}+\frac{Q^{2}}{r^{2}}} .
$$

The Faraday-Maxwell tensor, solution of Maxwell field equation in Eq. (2), is in terms of $Q$ given by

$$
F_{r t}=-F_{t r}=\frac{Q}{r^{2}},
$$

with the remaining components being identically null. When $\frac{Q}{M} \leq 1$ there are two important characteristic radii, the event horizon radius $r_{+}$and the Cauchy horizon $r_{-}$, with $M$ and $Q$ being given in terms of these by

$$
M=\frac{r_{+}+r_{-}}{2}, \quad Q=\sqrt{r_{+} r_{-}} .
$$


The line element Eq. (15) then represents a black hole solution and can written as

$$
d s^{2}=-\left(1-\frac{r_{+}}{r}\right)\left(1-\frac{r_{-}}{r}\right) d t^{2}+\frac{d r^{2}}{\left(1-\frac{r_{+}}{r}\right)\left(1-\frac{r_{-}}{r}\right)}+r^{2} d \Omega^{2},
$$

The redshift function of Eq. (16) at some radius $r$, is now $k\left(r, r_{+}, r_{-}\right)$given by

$$
k=\sqrt{\left(1-\frac{r_{+}}{r}\right)\left(1-\frac{r_{-}}{r}\right)} .
$$

The Faraday-Maxwell tensor of Eq. (17) is in terms of $r_{+}$and $r_{-}$given by

$$
F_{r t}=-F_{t r}=\frac{\sqrt{r_{+} r_{-}}}{r^{2}}
$$

Inverting Eq. (18) one obtains $r_{+}$and $r_{-}$in terms of the spacetime mass $M$ and the electric charge $Q$ as

$$
r_{+}=M+\sqrt{M^{2}-Q^{2}}, \quad r_{-}=M-\sqrt{M^{2}-Q^{2}} .
$$

When the solution is nonextremal, one has $r_{+}>r_{-}$, which in terms of $M$ and $Q$ is given by the condition $M^{2}>Q^{2}$, see Eq. (22), i.e., $\frac{Q}{M} \leq 1$. For the nonextremal solution we work with the line element as given in Eq. (19). A particular important instance here, is when $r_{-}=0$, i.e., the electric charge is zero, $Q=0, r_{+}=2 M$, and the Schwarzschild solution is recovered, $d s^{2}=-\left(1-\frac{r_{+}}{r}\right) d t^{2}+\frac{d r^{2}}{1-\frac{r_{+}}{r}}+r^{2} d \Omega^{2}$. For the extremal solution, one has $r_{+}=r_{-}$, which in terms of $M$ and $Q$ is given by the condition $M^{2}=Q^{2}$, see Eq. (22), i.e., $\frac{Q}{M}=1$. For the extremal solution we work with the line element as given in Eq. (19) putting $r_{+}=r_{-}$. For the overcharged solution, $r_{+}$and $r_{-}$take complex values, and in terms of $M$ and $Q$ it is given by the condition $M^{2}<Q^{2}$, see Eq. (22), i.e., $\frac{Q}{M}>1$, so for the overcharged solution it is definitely better to work not with the line element of Eq. (19) but with the line element of Eq. (15). The Reissner-Nordström spacetime, given by the line element Eq. (19), or Eq. (15), has a maximal extension. The Carter-Penrose diagrams for the three possible solutions, namely, nonextremal, extremal, and overcharged [40-42], are given in Figures 2, 3, and 4, respectively. Looking at the Carter-Penrose diagrams it is clear that the nonextremal, extremal, and overcharged Reissner-Nordström spacetimes have a very distinct causal structure. Let us look at them one at a time.

For the nonextremal solution, $r_{+}>r_{-}$, see Figure 2, the Carter-Penrose diagram shows that there is a central block that repeats itself. The central block is composed of regions I and I', regions II and II', and regions III and III'. Regions I and I' are delimited by the two null lines $r_{+}$, which are the event horizon of the spacetime, the past $\mathscr{I}^{-}$ and the future $\mathscr{I}^{+}$, the spacelike infinity $i_{0}$, and the timelike infinities $i^{-}$and $i^{+}$at the lower and upper vertices of the triangle that are not drawn to not overload the figure, regions II and II' are delimited by $r_{+}$and $r_{-}$, the event and the Cauchy horizons, respectively, and regions III and III' by $r_{-}$and the singularity at $r=0$. From region I to I' there is an Einstein-Rosen bridge, more precisely, a dynamic wormhole. Region II' is a white hole region, region II a black hole. Thus, in summary the central block is composed of a white and a black hole and two asymptotically flat regions connected by a wormhole that end in a region that contains Cauchy horizons which in turn can be past to the singularities at $r=0$. A detailed analysis of the causal structure and the construction of the diagram is given in Appendix A for the mathematics and properties of the maximal analytical extension of the vacuum nonextremal Reissner-Nordström solution. It will be seen that instead of the $(t, r)$ coordinates of Schwarzschild it is necessary to resort to $(T, X)$ coordinates of Kruskal to have a better hold on the extension.

For the extremal solution, $r_{+}=r_{-}$, see Figure 3, the Carter-Penrose diagram shows that there are no mirror regions, and so there is no Einstein-Rosen bridge, i.e., no dynamic wormhole. Region I is the asymptotically flat region, and region II is a black and white hole together.

For the overcharged solution, $r_{+}$and $r_{-}$do not exist, see Figure 4 , the Carter-Penrose diagram shows that there is only the asymptotic region with $r=0$ being both the origin of coordinates and a timelike singularity. 


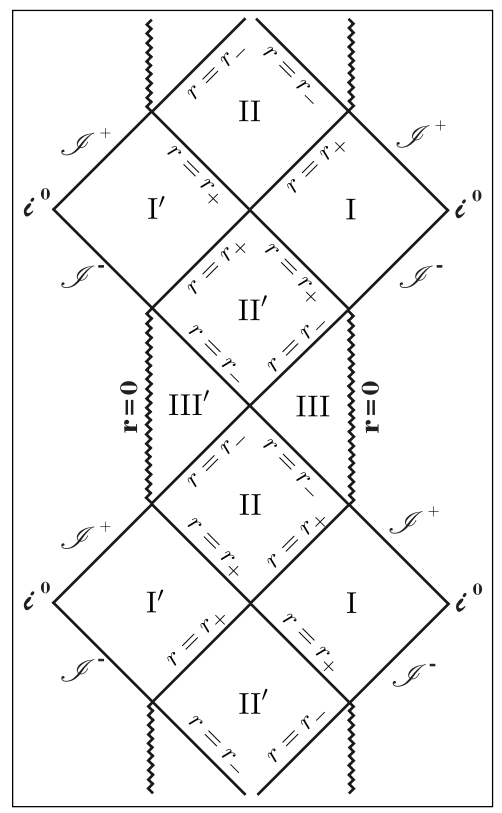

Figure 2: Carter-Penrose diagram of the nonextremal Reissner-Nordström spacetime. See text for details.

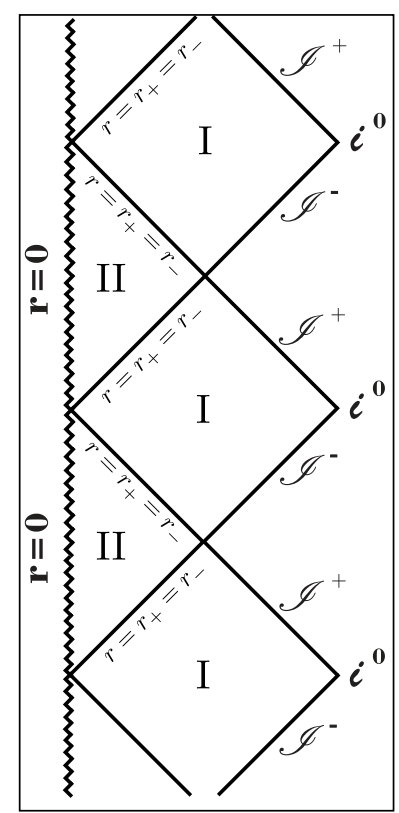

Figure 3: Carter-Penrose diagram of the extremal Reissner-Nordström spacetime. See text for details. 


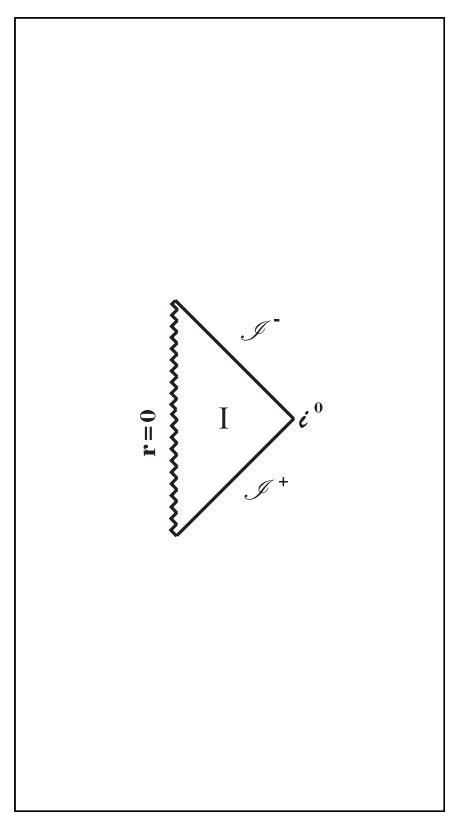

Figure 4: Carter-Penrose diagram of the overcharged Reissner-Nordström spacetime. See text for details.

\section{Classification scheme and nomenclature}

\section{Classification scheme}

Armed with the necessary formalism to make a junction of an interior Minkowski to an exterior Reissner-Nordström solution we can now proceed to find and classify all fundamental electrically charged static thin shells.

There are three distinct main electric states for the thin shell, namely, nonextremal, extremal, and overcharged. These yield an exterior Reissner-Nordström spacetime, that is nonextremal, i.e., $\frac{Q}{M}<1$ or $r_{+}>r_{-}$, extremal, i.e., $\frac{Q}{M}=1$ or $r_{+}=r_{-}$, and overcharged, i.e., $\frac{Q}{M}>1$, respectively.

For each of the three main states one has to assign a location for the shell, assumed to have radius $R$. In the nonextremal state, $r_{+}>r_{-}$, there are two possible locations, the shell can be located outside $r_{+}$, i.e., $R>r_{+}$, or inside $r_{-}$, i.e., $R<r_{-}$. In the extremal state, $r_{+}$and $r_{-}$coincide, $r_{+}=r_{-}$, there are three possible locations, the shell can be located outside $r_{+}$, i.e., $R>r_{+}$, inside $r_{+}$, i.e., $R<r_{+}$, or at $r_{+}$, i.e., $R=r_{+}$. In the overcharged state, there are no $r_{+}$and $r_{-}$, there is one generic location, the shell can be located anywhere without distinction, $R>0$.

For each of the locations one has to assign an orientation for the normal vector to the shell, indeed as seen by an external observer the normal vector to the shell can either point outward to increasing $r$ or point inward to decreasing $r$. Thus, in the nonextremal state with the shell located outside $r_{+}$, one can have either a shell with the orientation of the normal vector pointing to spatial infinity or one can have a shell with the orientation of the normal vector pointing to $r_{+}$. In the nonextremal state with the shell located inside $r_{-}$, one can have either a shell with the orientation of the normal vector pointing to $r_{-}$or one can have a shell with the orientation of the normal vector pointing to $r=0$. In the extremal state with the shell located outside $r_{+}$, one can have either a shell with the orientation of the normal vector pointing to spatial infinity or one can have a shell with the orientation of the normal vector pointing to $r=r_{+}$. In the extremal state with the shell located inside $r_{+}$, one can have either a shell with the orientation of the normal vector pointing to $r_{+}$or one can have a shell with the orientation of the normal vector pointing to $r=0$. In the extremal state with the shell located at $r_{+}$, one can have either a shell with the orientation of the normal vector pointing to spatial infinity or one can have a shell with the orientation of the normal vector pointing to $r=0$, and for each of these two orientations, there are two possible approaches, the shell approaches $r_{+}$from above, i.e., $R>r_{+}$ with $R \rightarrow r_{+}$, or the shell approaches $r_{+}$from below, i.e., $R<r_{+}$with $R \rightarrow r_{+}$. In the overcharged state, with the shell located anywhere, one can have either a shell with the orientation of the normal vector pointing to spatial infinity or one can have a shell with the orientation of the normal vector pointing to $r=0$.

So, the classification we perform for a fundamental electric thin shell and respective spacetime, namely, state, location, orientation, yields fourteen different cases. All the fourteen cases, four for nonextremal, eight for extremal, and two for overcharged will be analyzed, in particular, the physics and geometry of all the cases will be displayed. 


\section{Nomenclature}

There is a question of nomenclature that we must clarify. Note that the thin shell spacetime solution has a characteristic radius which is the radius $R$ of the location of the shell. For a shell in the nonextremal state, $r_{+}>r_{-}$, the exterior Reissner-Nordström exterior solution has two further characteristic radii, namely, $r_{+}$and $r_{-}$themselves. The question of nomenclature is the distinction between gravitational radius and event horizon radius on one hand, and between Cauchy radius and Cauchy horizon radius on the other. The gravitational radius and Cauchy radius of a nonextremal spacetime are a characteristic of the spacetime, more precisely, given a mass $M$ and charge $Q$ then there is a one-to-one correspondence to $r_{+}$and $r_{-}$. It can happen that the nonextremal shell spacetime has no horizons in which situation $r_{+}$and $r_{-}$are simply the gravitational radius and Cauchy radius of the spacetime, respectively, or it can happen that the nonextremal shell spacetime has horizons, in which situation the event horizon radius $r_{+}$is also the gravitational radius and the Cauchy horizon radius $r_{-}$is also the Cauchy radius. E.g., a nonextremal star object for which $\frac{Q}{M}<1$ has gravitational radius but no event horizon, since the spacetime in which it is inserted has no event horizon. For the same reason, it also has a Cauchy radius but no Cauchy horizon. On the other hand, a nonextremal black hole for which also $\frac{Q}{M}<1$ has the property that its gravitational radius is also its event horizon radius and its Cauchy radius is also its Cauchy horizon radius. For a shell in the extremal state, $r_{+}=r_{-}$, besides the radius $R$ of the shell, the exterior Reissner-Nordström exterior solution has one characteristic radius, namely, $r_{+}$, one could use $r_{-}$also but it is clearly more appropriate to use $r_{+}$. As before, depending on the location of the shell, $r_{+}$can be a gravitational radius alone when the extremal shell spacetime has no horizon, or it can be a gravitational radius and an event horizon radius as well, when the shell spacetime has a horizon. For a shell in the overcharged state, $r_{+}$and $r_{-}$are not defined, and so the nomenclature does not apply.

Let us see in detail this nomenclature when applied to the fundamental electric thin shells. First, the shell in the nonextremal state has four cases. If the radius $R$ of the shell is greater than $r_{+}$and its normal points towards spatial infinity, so the shell is located in region I of Figure 2, then there is no event horizon and no Cauchy horizon, and $r_{+}$ is the gravitational radius and $r_{-}$is the Cauchy radius. If the radius $R$ of the shell is greater than $r_{+}$and its normal points towards $r_{+}$, so the shell is located in region I' of Figure 2, then there are event and Cauchy horizons, and $r_{+}$is, in addition to a gravitational radius, an event horizon radius and $r_{-}$is also the Cauchy horizon radius. If the radius $R$ of the shell is less than $r_{-}$and its normal points towards $r_{-}$, so the shell is located in region III or III' of Figure 2, then $r_{+}$is the event horizon radius and $r_{-}$is the Cauchy horizon radius. If the radius $R$ of the shell is less than $r_{-}$ and its normal points towards the singularity $r=0$, so the shell is still located in region III or III' of Figure 2, then there is no event horizon and no Cauchy horizon, and $r_{+}$is the gravitational radius and $r_{-}$is the Cauchy radius. Second, the shell in the extremal state has eight cases. For the eight cases of the extremal state one has that, since $r_{+}=r_{-}$, there is no need for the name Cauchy which drops out, and the radius $r_{+}=r_{-}$is called gravitational radius and event horizon radius in the appropriate cases. Here one follows the nonextremal nomenclature, see also Figure 3 representing the shell's exterior region. Third, the shell in the overcharged state has two cases. The nomenclature in the overcharged state with Figure 4 representing the shell's exterior region, does not apply since $r_{+}$and $r_{-}$do not exist.

It is clearly convenient to use this nomenclature and to distinguish when there no horizons from when there are horizons. We follow it in the study of the fourteen different cases. 


\section{NONEXTREMAL ELECTRIC THIN SHELLS OUTSIDE THE GRAVITATIONAL RADIUS: STAR SHELLS AND TENSION SHELL BLACK HOLES}

\section{A. Nonextremal electric thin shells outside the gravitational radius: Star shells}

Here we study the case of a fundamental electric thin shell in the nonextremal state, i.e., $r_{+}>r_{-}$or $M>Q$, for which the shell's location obeys $R>r_{+}$, and for which the orientation is such that the normal to the shell points towards spatial infinity. In this case horizons do not exist and so, following the nomenclature, $r_{+}$and $r_{-}$are the gravitational radius and Cauchy radius, respectively. Two remarks are important. First, when we write $M>Q$ it is meant $M>|Q|$, but to simplify the notation, we drop the modulus in these instances, expecting that the context makes clear the meaning. Second, the normal to the shell pointing towards spatial infinity means in the notation of the Kruskal coordinate $X$ that we take $\operatorname{sign}(X)=+1$, see the end of this section and Appendix A for details.

As functions of $M, Q$, and $R$, the shell's energy density $\sigma$ and pressure $p$, are, see the end of this section,

$$
\begin{gathered}
8 \pi \sigma=\frac{2}{R}(1-k), \\
8 \pi p=\frac{1}{2 R k}\left[(1-k)^{2}-\frac{Q^{2}}{R^{2}}\right],
\end{gathered}
$$

with $k=\sqrt{1-\frac{2 M}{R}+\frac{Q^{2}}{R^{2}}}$. Also, the electric charge density $\sigma_{e}$ is given in terms of $M, Q$, and $R$, by

$$
8 \pi \sigma_{e}=\frac{2 Q}{R^{2}}
$$

explicitly showing that the shell's electric charge density is the source of the exterior Reissner-Nordström spacetime electric charge. The behavior of $\sigma$ and $p$ as functions of the radius $R$ of the shell for various values of the $\frac{Q}{M}$ ratio in this case is shown in Fig. 5. Since $M>Q$, and also $R>r_{+}$, and so $R>M$, one has that $\sigma$ and $p$, Eqs. (23)

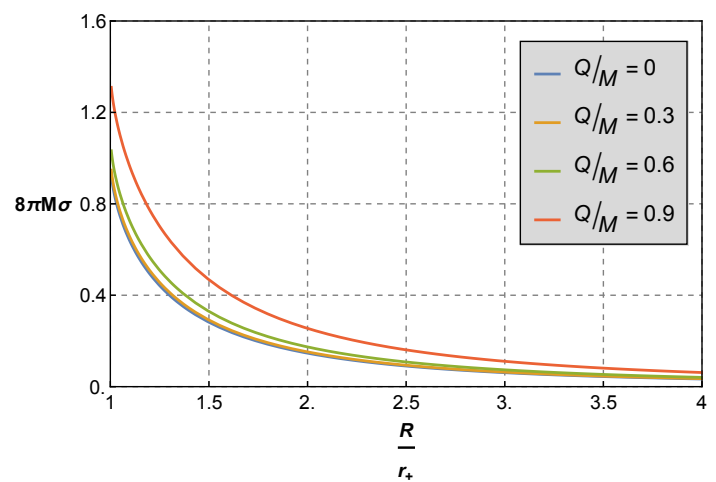

(a)

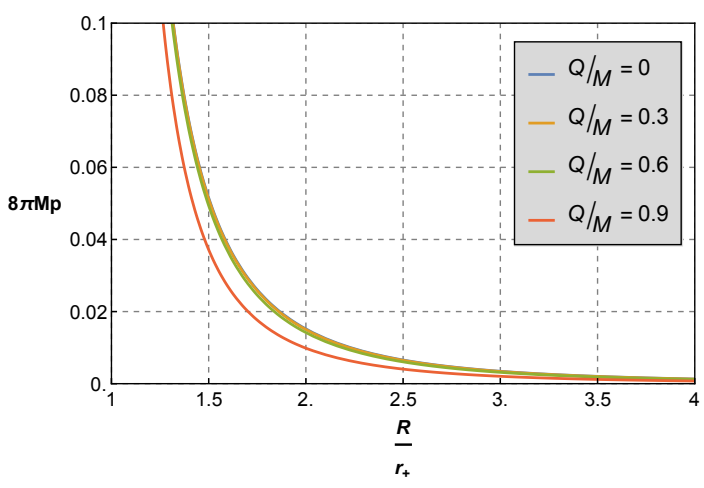

(b)

Figure 5: Physical properties of a nonextremal star shell, i.e., an electric perfect fluid thin shell in a nonextremal Reissner-Nordström state, in the location $R>r_{+}$, i.e., located outside the gravitational radius, and with orientation such that the normal points towards spatial infinity. The interior is Minkowski and the exterior is nonextremal Reissner-Nordström spacetime. Panel (a) Energy density $\sigma$ of the shell as a function of the radius $R$ of the shell for various values of the $\frac{Q}{M}$ ratio. The energy density is adimensionalized through the mass $M, 8 \pi M \sigma$, and the radius is adimensionalized through the gravitational radius $r_{+}, \frac{R}{r_{+}}$. Panel (b) Pressure $p$ on the shell as a function of the radius $R$ of the shell for various values of the $\frac{Q}{M}$ ratio. The pressure is adimensionalized through the mass $M$, $8 \pi M p$, and the radius is adimensionalized through the gravitational radius $r_{+}, \frac{R}{r_{+}}$.

and (24), are always positive for this type of shells, as can be also checked in Fig. 5. These are the star shells. Qualitatively, one can understand why the pressure is positive for shells with normal pointing towards spatial infinity, i.e., $\operatorname{sign}(X)=+1$. A free-falling particle in the region outside the shell sees a geometry that is indistinguishable from the nonextremal Reissner-Nordström spacetime. Therefore, a particle, momentarily comoving with the shell but 
detached from it say, would tend to fall to the inside as if an event horizon existed. Therefore, in order to be static, a thin shell located at the junction hypersurface with $R>r_{+}$must by supported by pressure. Notice from Fig. 5 that as the charge $Q$ is increased one needs less pressure support, as expected, the electric repulsion makes up for the pressure. Notice that when $R \rightarrow \infty$, the energy density $\sigma$, the pressure $p$, and the charge density $\sigma_{e}$, all tend to zero, i.e., the shell disperses away. Notice also that when $R \rightarrow r_{+}$, the energy density is finite, but the pressure of the shell goes to infinity, while the electric charge density is also finite. Indeed, for $R=r_{+}$one has a quasiblack hole. When $Q=0$ the outer solution is Schwarzschild. In relation to the energy conditions of the shell one can work out and find that the null and the weak energy conditions are verified for $R>r_{+}$, the dominant energy condition for $R \geq R_{\mathrm{I}}$, where $R_{\mathrm{I}}$ is some specific radius that we present later, and the strong energy condition for $R>r_{+}$, see a detailed presentation ahead.

The Carter-Penrose diagram for this case can be drawn directly from the building blocks of an interior Minkowski spacetime and the exterior asymptotic infinite region of the nonextremal Reissner-Nordström spacetime. In Fig. 6 the Carter-Penrose diagram of a shell spacetime in a nonextremal Reissner-Nordström state, which includes Schwarzschild, in the location $R>r_{+}$, with orientation such that the normal points towards spatial infinity, i.e., $\operatorname{sign}(X)=+1$, is shown. It is clearly a star shell, a star in an asymptotically flat spacetime.

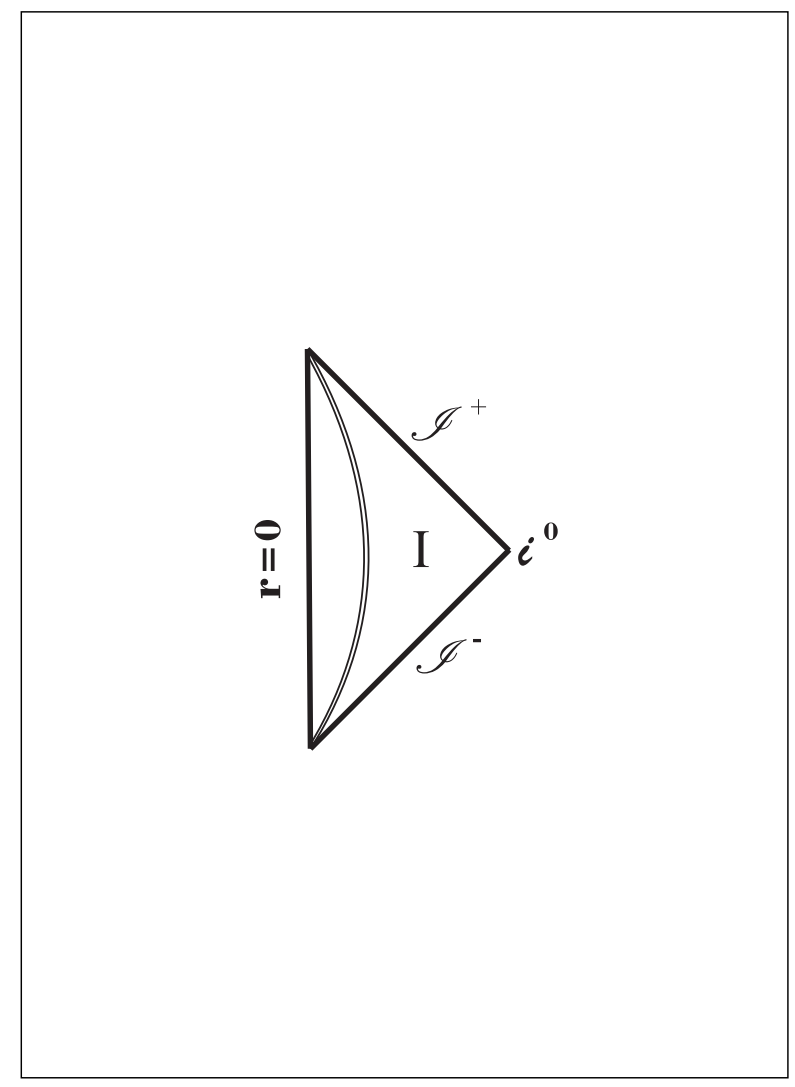

Figure 6: Carter-Penrose diagram of a star shell, i.e., a thin shell spacetime in a nonextremal Reissner-Nordström state, in the location $R>r_{+}$, i.e., located outside the gravitational radius, with orientation such that the normal points towards spatial infinity. The interior is Minkowski, the exterior is nonextremal Reissner-Nordström. For zero electric charge the exterior is Schwarzschild, in which case the diagram looks the same.

The physical interpretation of this case is clear cut. This nonextremal thin shell solution mimics a familiar star. The energy density and pressure obey the energy conditions if the radius of the shell is sufficiently large. When this radius approaches the gravitational radius the energy conditions are not obeyed, and at the gravitational radius itself the solution turns into a quasiblack hole an object with very interesting properties. The causal and global structure as displayed by the Carter-Penrose diagram are well behaved and rather elementary. So, this case falls into the category of having the energy conditions verified and the geometrical setup is physically reasonable. 


\section{B. Nonextremal electric thin shells outside the event horizon: Tension shell black holes}

Here we study the case of a fundamental electric thin shell in the nonextremal state, i.e., $r_{+}>r_{-}$or $M>Q$, for which the shell's location obeys $R>r_{+}$, and for which the orientation is such that the normal to the shell points towards $r_{+}$. In this case horizons do exist and so, following the nomenclature, $r_{+}$is both the gravitational and the event horizon radius, and $r_{-}$is both the Cauchy radius and the Cauchy horizon radius. The normal to the shell pointing towards $r_{+}$means in the notation of the Kruskal coordinate $X$ that we take $\operatorname{sign}(X)=-1$, see the end of this section and Appendix A for details.

As functions of $M, Q$, and $R$, the shell's energy density $\sigma$ and pressure $p$, are, see the end of this section,

$$
\begin{gathered}
8 \pi \sigma=\frac{2}{R}(1+k), \\
8 \pi p=-\frac{1}{2 R k}\left[(1+k)^{2}-\frac{Q^{2}}{R^{2}}\right],
\end{gathered}
$$

where the redshift parameter $k$ is again $k=\sqrt{1-\frac{2 M}{R}+\frac{Q^{2}}{R^{2}}}$. The electric charge density $\sigma_{e}$ is given in terms of $M, Q$, and $R$ also by Eq. (25). The behavior of $\sigma$ and $p$ as functions of the radial coordinate $R$ of the shell for various values of the $\frac{Q}{M}$ ratio in this case is shown in Fig. 7. We see that $\sigma$ is always positive but $p$ is negative, it is rather a tension. These are the tension shells. Qualitatively, one can understand why these shells, with normal pointing to $r_{+}$, i.e., $\operatorname{sign}(X)=-1$, must be supported by tension, by remembering that a free-falling particle in the region outside the event horizon will infall towards the event horizon $r_{+}$itself. Therefore, a particle momentarily comoving with the shell but detached from it will infall towards the black hole region of the exterior Reissner-Nordström spacetime, hence, a perfect fluid thin shell located at the junction hypersurface, in order to be static, must by supported by tension. Notice from Fig. 7 that as the charge $Q$ is increased one needs more tension support, as expected, the electric repulsion obliges an increase in the tension. Notice that here $R$ is finite, although it can be arbitrarily large, in which case the energy density $\sigma$, the tension $-p$, and the charge density $\sigma_{e}$, all tend to zero. Notice that $\sigma$ has a nonmonotonic behavior. Notice also that when $R \rightarrow r_{+}$, the energy density is finite, but the tension of the shells goes to infinity, while the charge density is also finite. Indeed, for $R=r_{+}$one has a shell at the horizon with properties similar to a quasiblack hole, although one with additional structures. When $Q=0$ the outer solution is Schwarzschild. In relation to the energy conditions of the shell one can work out and find that the null, the weak, and the dominant energy conditions are verified for $R \geq R_{I^{\prime}}$, where $R_{I^{\prime}}$ is some specific radius that we present later, and the strong energy condition is never verified, see a detailed presentation ahead. 


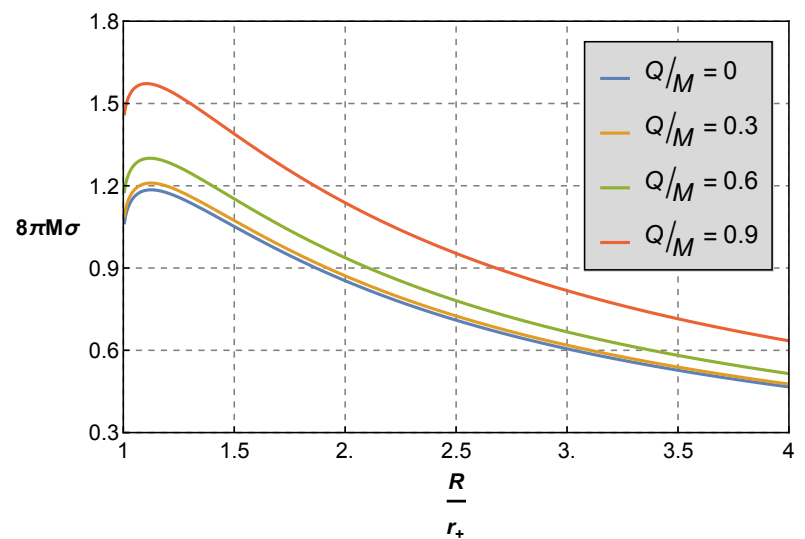

(a)

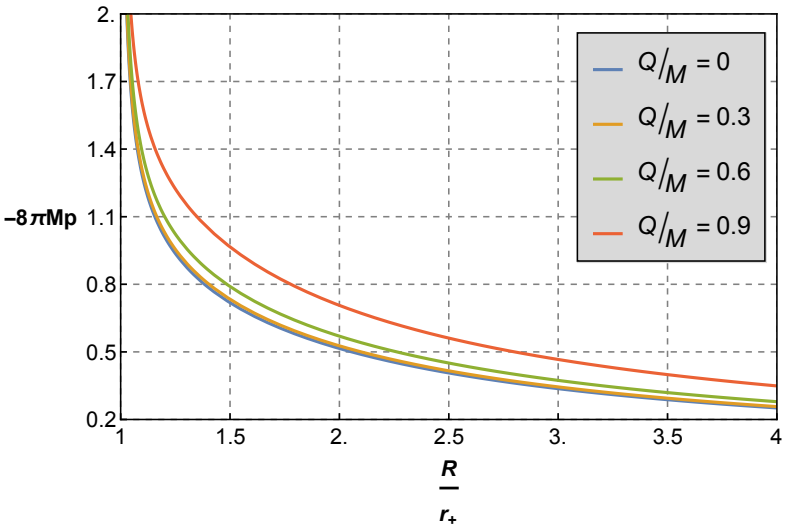

(b)

Figure 7: Physical properties of a nonextremal tension shell black hole, i.e., an electric perfect fluid thin shell in a nonextremal Reissner-Nordström state, in the location $R>r_{+}$, i.e., located outside the event horizon, with orientation such that the normal points towards $r_{+}$. The interior is Minkowski, the exterior is nonextremal Reissner-Nordström spacetime. Panel (a) Energy density $\sigma$ of the shell as a function of the radius $R$ of the shell for various values of the $\frac{Q}{M}$ ratio. The energy density is adimensionalized through the mass $M, 8 \pi M \sigma$, and the radius is adimensionalized through the gravitational radius $r_{+}, \frac{R}{r_{+}}$. Panel (b) Tension $-p$ on the shell as a function of the radius $R$ of the shell for various values of the $\frac{Q}{M}$ ratio. The tension is adimensionalized through the mass $M$, $-8 \pi M p$, and the radius is adimensionalized through the gravitational radius $r_{+}, \frac{R}{r_{+}}$.

The Carter-Penrose diagram for this case can be drawn from the building blocks of an interior Minkowski spacetime and the full nonextremal Reissner-Nordström spacetime. In Fig. 8 the Carter-Penrose diagram of a shell spacetime in a nonextremal Reissner-Nordström state, in the location $R>r_{+}$, with orientation such that the normal points towards $r_{+}$, i.e., $\operatorname{sign}(X)=-1$, is shown. In the diagram it is clear that the tension shell is in the other side of the Carter-Penrose diagram of a Reissner-Nordström spacetime. From Fig. 8 it is seen, that it is clearly a black hole solution, not a vacuum black hole, neither a regular black hole. The solutions represent tension shell black holes. Note $r_{+}$and $r_{-}$are the event horizon and the Cauchy horizon radii, and there is an Einstein-Rosen bridge, provided by a dynamic wormhole in the spacetime. Tension shell black holes were found in [12] for the zero electric charge case, i.e., for the Schwarzschild shells, in which case the Carter-Penrose diagram is similar, only the $r=0$ singularity is spacelike, and the diagram does not repeat itself. In the Reissner-Nordström spacetime, contrary to Schwarzschild, there is an infinitude of possible diagrams. In the diagram (a) of Fig. 8 it is clear that the tension shell is outside the event horizon in the other side of the diagram in the region $\mathrm{I}^{\prime}$ shown. One can then put another shell in the region $\mathrm{I}^{\prime}$ above and repeating the procedure ad infinitum. In the diagram (b) of Fig. 8 the tension shell is again outside the event horizon in the other side of the diagram in the region $\mathrm{I}^{\prime}$ shown. One can then put an infinity region in the region $\mathrm{I}^{\prime}$ above and repeating the procedure ad infinitum. As what one puts in the regions $\mathrm{I}^{\prime}$, either a tension shell or infinity, is not decided by the solution, an infinite number of different Carter-Penrose diagrams can be drawn, since there are an infinite number of combinations to locate a shell or infinity when one goes upward or downward through the diagram. When $R=r_{+}$the shell with its interior forms a tension quasiblack hole with special features since it is attached to the other regions of the Reissner-Nordström spacetime. 


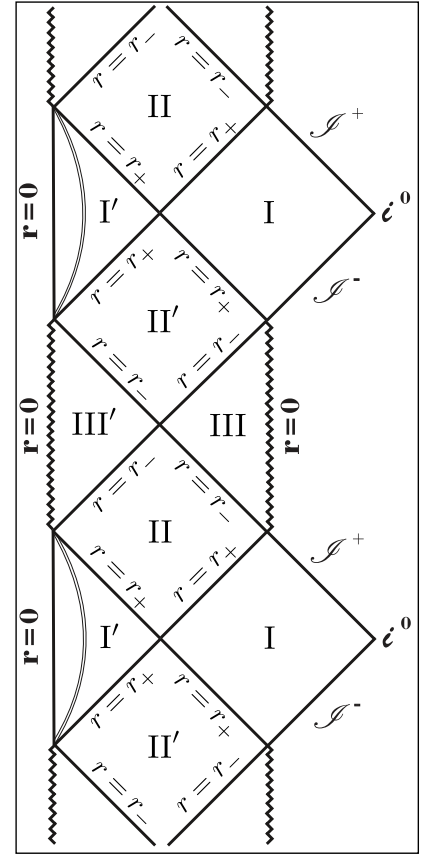

(a)

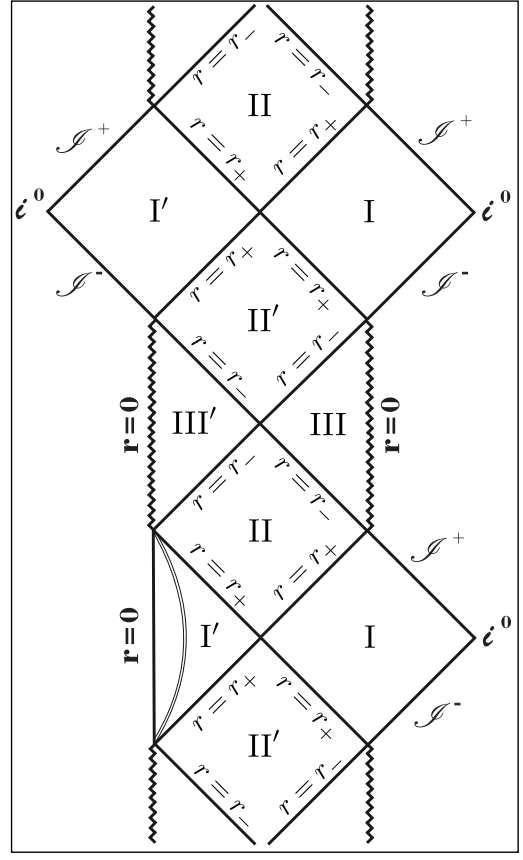

(b)

Figure 8: Carter-Penrose diagrams of a tension shell black hole, i.e., a thin shell spacetime in a nonextremal Reissner-Nordström state, in the location $R>r_{+}$, i.e., located outside the event horizon, with orientation such that the normal points towards $r_{+}$. The interior is Minkowski, the exterior is nonextremal Reissner-Nordström spacetime. For zero electric charge the exterior is Schwarzschild, in which case the timelike singularities turn into spacelike ones. Panel (a) The Carter-Penrose diagram contains a shell in the regions I' shown and another shell in the next region

$\mathrm{I}^{\prime}$, which is repeated for all regions $\mathrm{I}^{\prime}$. Panel (b) The Carter-Penrose diagram contains a shell in region $\mathrm{I}^{\prime}$ and an infinity in the next region $\mathrm{I}^{\prime}$, which is then repeated for all regions $\mathrm{I}^{\prime}$. An infinite number of different Carter-Penrose diagrams can be drawn, since there are an infinite number of combinations to locate the shell and infinity.

The physical interpretation of this case has some complexity. This nonextremal thin shell solution carries with it a white hole connected to a black hole through a wormhole. The energy density and pressure obey some of the energy conditions if the radius of the shell is sufficiently large, i.e., is sufficiently larger than the gravitational radius. When the radius of the shell approaches the gravitational radius, the energy conditions are not obeyed, and when the radius of the shell is at the gravitational radius the solution turns into a tension quasiblack hole an object with interesting properties. The causal and global structures as displayed by the Carter-Penrose diagram in its simplest form shows the important spacetime regions. We have called this solution a tension shell black hole, but it could be called as well a tension shell nontraversable wormhole, since there is a nontraversable wormhole that links the white hole to the black hole region. As in Reissner-Nosdström solution, This tension shell black hole possesses Cauchy horizons, and, as in the vacuum Reissner-Nosdström solution, it is subject to be destroyed by perturbations. Presumably, the perturbation would turn the Cauchy horizon into a null or spacelike singularity, turning in turn the nonextremal tension shell solution into a solution similar to the electrically uncharged Lynden-Bell-Katz tension shell black hole solution. Moreover, these solutions, in the same way as the full Reissner-Nosdström or Schwarzschild solutions, are universes in themselves, and, if they existed, they would have to be given directly by mother nature, rather than appear by, say, a straight gravitational collapse or some other process. So, this case falls into the category of having some of the energy conditions verified and the geometrical setup being physically peculiar, although full of interest, as matter solutions on the other side of the Carter-Penrose diagram are rare. Moreover these solutions are familiar, in the sense that nontraversable wormholes with white and black holes are well known. 


\section{Formalism for nonextremal electric thin shells outside the gravitational radius}

\section{Preliminaries}

We now make a careful study to derive the properties of the fundamental electric thin shell used in the two previous subsections, i.e., the thin shell in the nonextremal state, i.e., $r_{+}>r_{-}$or $M>Q$, for which the shell's location obeys $R>r_{+}$, and for which the orientation is such that the normal to the shell points towards spatial infinity or towards $r_{+}$. It should be read as an appendix to the previous two subsections. We use the formalism developed in Sec. II and Appendix A.

\section{Induced metric and extrinsic curvature of $\mathcal{S}$ as seen from $\mathcal{M}_{\mathrm{i}}$}

Let us start by analyzing the interior Minkowski spacetime, $\mathcal{M}_{\mathrm{i}}$, whose line element in spherical coordinates is given by

$$
d s_{\mathrm{i}}^{2}=-d t_{\mathrm{i}}^{2}+d \mathrm{r}^{2}+\mathrm{r}^{2} d \Omega^{2}
$$

where $t_{\mathrm{i}}$ and $\mathrm{r}$ are the time and radial coordinates, respectively, and $d \Omega^{2} \equiv d \theta^{2}+\sin ^{2} \theta d \varphi^{2}$, with $\theta$ and $\varphi$ being the angular coordinates. The subscript i denotes interior or inside from now onwards.

The junction from the interior to the exterior is made through a hypersurface $\mathcal{S}$. We assume the hypersurface $\mathcal{S}$ to be static, i.e., static as seen from a free-falling observer in the interior Minkowski spacetime. In general, $\mathcal{S}$ can be either timelike or spacelike, however, since we are considering Minkowski spacetime, it is not possible to have a static spacelike surface hence, $\mathcal{S}$ must be timelike. It is convenient to choose the coordinates on $\mathcal{S}$ to be $\left\{y^{a}\right\}=(\tau, \theta, \varphi)$, where $\tau$ is the proper time measured by an observer comoving with $\mathcal{S}$. It follows that denoting $u_{\mathrm{i}}$ as the 4 -velocity of an observer comoving with the shell as seen from the inside, we can define a unit vector $e_{\tau}$ such that $e_{\tau} \equiv u_{\mathrm{i}}$. The hypersurface $\mathcal{S}$, as seen from the interior spacetime $\mathcal{M}_{\mathrm{i}}$, is parameterized by $\tau$, such that the surface's radial coordinate is described by a function $\left.\mathrm{r}\right|_{\mathcal{S}} \equiv R=R(\tau)$. The fact that $\mathcal{S}$ is assumed to be static implies $\frac{d R}{d \tau}=0$, from which we have that $u_{\mathrm{i}}^{\alpha}=\left(\frac{d t_{\mathrm{i}}}{d \tau}, 0,0,0\right)$, where $u_{\mathrm{i}}^{\alpha}$ represents the components of the 4 -velocity $u_{\mathrm{i}}$ as seen from the interior spacetime $\mathcal{M}_{\mathrm{i}}$. Since $\mathcal{S}$ is a timelike hypersurface, it must verify $u_{\mathrm{i} \alpha} u_{\mathrm{i}}^{\alpha}=-1$. With these latter two equations we find that $\frac{d t_{\mathrm{i}}}{d \tau}= \pm 1$. Imposing that $u_{\mathrm{i}}$ points to the future leads to the choice of the plus sign, thus

$$
u_{\mathrm{i}}^{\alpha}=(1,0,0,0)
$$

From Eqs. (5) and (29) we can find the induced metric on $\mathcal{S}$ by the spacetime $\mathcal{M}_{\mathrm{i}}$, such that

$$
\left.d s_{\mathrm{i}}^{2}\right|_{\mathcal{S}}=-d \tau^{2}+R^{2} d \Omega^{2}
$$

Also, with the expression for the 4 -velocity of an observer comoving with $\mathcal{S}$, we can now use Eqs. (4) and (29) to find the expression for the components of the unit normal as seen from $\mathcal{M}_{\mathrm{i}}$, $n_{\mathrm{i}}^{\alpha}$, hence $n_{\mathrm{i} \alpha}=\lambda(0,1,0,0)$ where $\lambda$ is a normalization factor. Using Eqs. (3) and (28) and the condition that $n$ is spacelike, yields $\lambda= \pm 1$. Since we are studying the case where the interior Minkowski spacetime is spatially compact and enclosed by the hypersurface $\mathcal{S}$, we must choose the plus sign, such that, the expression for the outward pointing unit normal to $\mathcal{S}$ is given by

$$
n_{\mathrm{i} \alpha}=(0,1,0,0)
$$

We are now in position to compute the components of the extrinsic curvature of $\mathcal{S}$ as seen from $\mathcal{M}_{\mathrm{i}}, K_{\mathrm{i} a b}$. In the case where the matching surface $\mathcal{S}$ is timelike, static and spherically symmetric, the nonzero components of the extrinsic curvature are given by $K_{\tau \tau}=-a^{\alpha} n_{\alpha}, K_{\theta \theta}=\nabla_{\theta} n_{\theta}, K_{\varphi \varphi}=\nabla_{\varphi} n_{\varphi}$, where $a^{\alpha} \equiv u^{\beta} \nabla_{\beta} u^{\alpha}$, see Appendix B. Taking into account Eqs. (4), (28), (30), and (31), we find that the nontrivial components of the extrinsic curvature as seen from the interior Minkowski spacetime, see Eq. (6), are given by

$$
K_{\mathrm{i}}^{\tau} \tau=0, \quad K_{\mathrm{i}}^{\theta}{ }^{\theta}=K_{\mathrm{i}}^{\varphi} \varphi=\frac{1}{R}
$$

where the induced metric taken from Eq. (30) was used to raise the indices. 


\section{Induced metric and extrinsic curvature of $\mathcal{S}$ as seen from $\mathcal{M}_{\mathrm{e}}$}

To proceed we have now to find the expressions for the induced metric on $\mathcal{S}$ and the extrinsic curvature components as seen from the exterior spacetime, $\mathcal{M}_{\mathrm{e}}$, in the nonextremal state, i.e., $r_{+}>r_{-}$or $M>Q$, see Figure 2 , for which the shell's location obeys $R>r_{+}$, and for which the orientation is such that the normal to the shell points towards increasing $r$ or towards decreasing $r$ as seen from the exterior, as used in the two previous subsections.

For a nonextremal shell with $R>r_{+}$we work with the coordinate pathch that has no coordinate singularity at the gravitational radius $r=r_{+}$. For the setting of coordinate patches in the nonextremal Reissner-Nordström spacetime see Appendix A, see also [13] for the coordinate patches of an uncharged shell matched to the Schwarzschild spacetime. In this region and for the chosen coordinate patch, the line element for the Reissner-Nordström spacetime in KruskalSzekeres coordinates is given by

$$
\begin{gathered}
d s_{\mathrm{e}}^{2}=4\left(\frac{r_{+}+r_{-}}{r_{+}-r_{-}}\right)^{2} \frac{r_{+}^{4}}{r^{2}} e^{-\frac{r\left(r_{+}-r_{-}\right)}{r_{+}^{2}}}\left(\frac{r-r_{-}}{r_{+}+r_{-}}\right)^{1+\left(\frac{r_{-}}{r_{+}}\right)^{2}}\left(d X^{2}-d T^{2}\right)+r^{2}(T, X) d \Omega^{2}, \\
X^{2}-T^{2}=e^{\frac{r\left(r_{+}-r_{-}\right)}{r_{+}^{2}}}\left(\frac{r-r_{+}}{r_{+}+r_{-}}\right)\left(\frac{r-r_{-}}{r_{+}+r_{-}}\right)^{-\left(\frac{r_{-}}{r_{+}}\right)^{2}},
\end{gathered}
$$

with $r(T, X)$ being given implicitly by the latter equation. The subscript e denotes exterior from now onwards.

The shell's radial coordinate when measured by an observer at $\mathcal{M}_{\mathrm{e}}$ is described by a function $\left.r\right|_{\mathcal{S}} \equiv R=R(\tau)$, where $\tau$ is the proper time of an observer comoving with the surface $\mathcal{S}$, which, since we assume it to be static, is such that $\frac{d R}{d \tau}=0$. Strictly, $R$ should be written as another letter, say $\mathcal{R}$, but as we will see we can put $\mathcal{R}=R$ and so we stick to the letter $R$ from the start. Considering the second of the equations given in Eq. (33), $\frac{d R}{d \tau}=0$ implies that the $X$ and $T$ coordinates of a point on $\mathcal{S}$ must verify $X^{2}-T^{2}=$ constant. Taking the derivative of $X^{2}-T^{2}=$ constant in order to the proper time we find the relation $\frac{\partial X}{\partial \tau}=\frac{T}{X} \frac{\partial T}{\partial \tau}$. In our previous analysis of the $\mathcal{M}_{\mathrm{i}}$ spacetime, we found that the hypersurface $\mathcal{S}$ must be timelike, then, due to the first junction condition, $\mathcal{S}$ must also be timelike when seen from the exterior $\mathcal{M}_{\mathrm{e}}$ spacetime. Therefore, the components of the 4-velocity of an observer comoving with it as seen from $\mathcal{M}_{\mathrm{e}}$ are, $u_{\mathrm{e}}^{\alpha}=\left(\frac{\partial T}{\partial \tau}, \frac{\partial X}{\partial \tau}, 0,0\right)$. Using $\frac{\partial X}{\partial \tau}=\frac{T}{X} \frac{\partial T}{\partial \tau}$ and $u_{\mathrm{e} \alpha} u_{\mathrm{e}}^{\alpha}=-1$ we find $\frac{\partial T}{\partial \tau}= \pm \sqrt{\frac{g^{X X} X^{2}}{X^{2}-T^{2}}}$ and $\frac{\partial X}{\partial \tau}= \pm \sqrt{\frac{g^{X X} T^{2}}{X^{2}-T^{2}}}$, so that,

$$
u_{\mathrm{e}}^{\alpha}=\sqrt{\frac{g^{X X}}{X^{2}-T^{2}}}(X, T, 0,0),
$$

where the sign was chosen in order that $u_{\mathrm{e}}$ points to the future and $g^{x x}$ is the $X X$ component of the inverse metric associated with Eq. (33). Notice that the expression found for the components of $u_{\mathrm{e}}$, Eq. (34), only makes sense, physically, if $X^{2}>T^{2}$. Looking at the second of the equations given in Eq. (33), one has that $X^{2}>T^{2}$ implies that $R>r_{+}$so, either the shell is located in the region $\mathrm{I}$ or in the region $\mathrm{I}^{\prime}$, see Figure 2 . The restriction on the allowed regions for the shell is a consequence of the shell being assumed static, if we were to consider a dynamic shell or a different interior spacetime, then shells in the black hole or the white hole region could also be treated. Note also that our choice of the plus sign in Eq. (34), such that $u_{\mathrm{e}}$ points to the future, is the correct one in both I or I' regions. Equation (34) can now be used to find the induced metric on the hypersurface $\mathcal{S}$ by the spacetime $\mathcal{M}_{\mathrm{e}}$, such that $\left.d s_{\mathrm{e}}^{2}\right|_{\mathcal{S}}=-d \tau^{2}+R^{2} d \Omega^{2}$. From the first junction condition, Eq. (8), matching Eq. (30) with this equation for $\left.d s_{\mathrm{e}}^{2}\right|_{\mathcal{S}}$, we find that $R$, the radial coordinate of $\mathcal{S}$ when measured by an observer at $\mathcal{M}_{\mathrm{e}}$, and $R$, the radial coordinate of $\mathcal{S}$ when measured by an observer at $\mathcal{M}_{\mathrm{i}}$, must be indeed equal, as we have anticipated. So, generically, $R$ describes the radial coordinate of the shell for either the interior and exterior spacetime, and so, the intrinsic line elements of the shell, namely, $\left.d s_{\mathrm{i}}^{2}\right|_{\mathcal{S}}=-d \tau^{2}+R^{2} d \Omega^{2}$ and $\left.d s_{\mathrm{e}}^{2}\right|_{\mathcal{S}}=-d \tau^{2}+R^{2} d \Omega^{2}$, can be written uniquely as

$$
\left.d s^{2}\right|_{\mathcal{S}}=-d \tau^{2}+R^{2} d \Omega^{2}
$$

Now, using the fact that the unit normal to $\mathcal{S}$ is spacelike, implies $n_{\mathrm{e}}^{\alpha} n_{\mathrm{e} \alpha}=+1$. Then, taking into account Eqs. (4) and (34), we find $n_{\mathrm{e} \alpha}= \pm \sqrt{\frac{g_{X X}}{X^{2}-T^{2}}}(-T, X, 0,0)$. To proceed, we must choose the sign for the normal. The choice of the sign is related with the orientation of the shell, i.e., the direction of the normal, and we impose that it points in the direction of increasing $X$ coordinate. This implies that the choice of the sign is different if we consider the shell to be in the region I or I', see Figure 2 and also Figure A1 of Appendix A 1. One of the simplifications that the use of the Kruskal-Szekeres coordinates introduces is that the choice of the sign can be written in a concise manner, such that

$$
n_{\mathrm{e} \alpha}=\operatorname{sign}(X) \sqrt{\frac{g_{X X}}{X^{2}-T^{2}}}(-T, X, 0,0)
$$


where the quantities on the right-hand side are to be evaluated at $r=R$ and $\operatorname{sign}(X)$ is the signum function of the coordinate $X$ of the shell. Notice however, that the usage of this notation is simply to treat in a concise way the two possible directions of the normal of the shell. Physically, there is nothing different between a shell located in either region, i.e., with positive or negative values of $X$. Having found the normal to the hypersurface $\mathcal{S}$ as seen from the exterior nonextremal Reissner-Nordström spacetime, we can now compute the nonzero components of the extrinsic curvature. Following the results in Appendix B 1 we have

$$
K_{\mathrm{e}}{ }^{\tau} \tau=\frac{\operatorname{sign}(X)}{2 R^{2} k}\left(r_{+}+r_{-}-2 \frac{r_{+} r_{-}}{R}\right), \quad K_{\mathrm{e}}{ }^{\theta}{ }_{\theta}=K_{\mathrm{e}}{ }_{\varphi} \varphi=\frac{\operatorname{sign}(X)\left(r_{+}-r_{-}\right)}{2 r_{+}^{2} R} \sqrt{g_{X X}\left(X^{2}-T^{2}\right)},
$$

where $k$, here, is the redshift function given in Eq. (20), evaluated at $R$, i.e., $k\left(R, r_{+}, r_{-}\right)=\sqrt{\left(1-\frac{r_{+}}{R}\right)\left(1-\frac{r_{-}}{R}\right)}$.

\section{Shell's energy density and pressure}

We are now in position to find the properties of a perfect fluid thin shell in a nonextremal Reissner-Nordström state, located outside the gravitational radius or event horizon radius, depending on the case. The shell's stress-energy tensor is given in Eq. (10), an expression containing the energy per unit area $\sigma$, the tangential pressure of the fluid $p$, the velocity $u_{a}$, and the induced metric $h_{a b}$. From our choice of coordinates on $\mathcal{S}$ we have that $\left\{y^{a}\right\}=(\tau, \theta, \varphi)$, the four-velocity $u_{\mathrm{i}}^{\alpha}$ is given in Eq. (29), and the metric $h_{a b}$ is given through Eq. (35). Putting everything together we find that $S_{\tau}^{\tau}=-\sigma, S_{\theta}^{\theta}=S_{\varphi}^{\varphi}=p$. Comparing these latter equations with the second junction condition, Eq. (9), taking into account the components of the induced metric, given through Eq. (35), and the fact that $\left[K_{\theta}^{\theta}\right]=\left[K_{\varphi}^{\varphi}\right]$, we find $\sigma=-\frac{1}{4 \pi}\left[K_{\theta}^{\theta}\right]$ and $p=\frac{1}{8 \pi}\left[K_{\tau}^{\tau}\right]-\frac{\sigma}{2}$. With the components of the extrinsic curvature found in Eqs. (32) and (37) we then obtain

$$
\begin{gathered}
8 \pi \sigma=\frac{2}{R}(1-\operatorname{sign}(X) k), \\
8 \pi p=\frac{\operatorname{sign}(X)}{2 R k}\left[(1-\operatorname{sign}(X) k)^{2}-\frac{r_{+} r_{-}}{R^{2}}\right],
\end{gathered}
$$

where $k$ here is the redshift function given in Eq. (20) evaluated at $R$, i.e., $k\left(R, r_{+}, r_{-}\right)=\sqrt{\left(1-\frac{r_{+}}{R}\right)\left(1-\frac{r_{-}}{R}\right)}$. As the surface electric current density $s_{a}$ on the thin shell is defined as $s_{a}=\sigma_{e} u_{a}$, where $\sigma_{e}$ represents the electric charge density and $u_{a}$ is the velocity of the shell, and since the Minkowski spacetime has zero electric charge, from Eqs. (12)-(13) and (21) it follows that

$$
8 \pi \sigma_{e}=2 \frac{\sqrt{r_{+} r_{-}}}{R^{2}}
$$

In Eqs. (38) and (39) it is clear that it is necessary to pick the sign in $\operatorname{sign}(X)$. Let us start with sign $(X)=+1$. It is useful here to give the expressions for the shell's energy density and pressure, $\sigma$ and $p$, in terms of $M$ and $Q$. Using Eq. (22) in Eqs. (38) and (39) with $\operatorname{sign}(X)=+1$ we have $8 \pi \sigma=\frac{2}{R}(1-k), 8 \pi p=\frac{1}{2 R k}\left[(1-k)^{2}-\frac{Q^{2}}{R^{2}}\right]$, where $k(R, M, Q)=\sqrt{1-\frac{2 M}{R}+\frac{Q^{2}}{R^{2}}}$, and also from Eq. (40) we have $8 \pi \sigma_{e}=\frac{2 Q}{R^{2}}$. Let us now take $\operatorname{sign}(X)=-1$. It is also useful here to give the expressions for the shell's energy density and pressure, $\sigma$ and $p$, in terms of $M$ and $Q$. Using Eq. (22) in Eqs. (38) and (39) with $\operatorname{sign}(X)=-1$ we have $8 \pi \sigma=\frac{2}{R}(1+k), 8 \pi p=-\frac{1}{2 R k}\left[(1+k)^{2}-\frac{Q^{2}}{R^{2}}\right]$, where again $k(R, M, Q)=\sqrt{1-\frac{2 M}{R}+\frac{Q^{2}}{R^{2}}}$, and also from Eq. (40) we have $8 \pi \sigma_{e}=\frac{2 Q}{R^{2}}$. These are the expressions used in the two previous subsections. Note also that when $r_{-}=0$, then $\sigma_{e}=0$ and the electric charge $Q$ is zero, $Q=0$, so the outside spacetime is described by the Schwarzschild solution, for which Eqs. (38) and (39) can be written explicitly as $\left.8 \pi \sigma\right|_{r_{-}=0}=\frac{2}{R}\left(1-\operatorname{sign}(X) \sqrt{1-\frac{r_{+}}{R}}\right)$ and $\left.8 \pi p\right|_{r_{-}=0}=\frac{\operatorname{sign}(X)}{2 R} \sqrt{\frac{1}{1-\frac{r_{+}}{R}}}\left[\left(1-\operatorname{sign}(X) \sqrt{1-\frac{r_{+}}{R}}\right)^{2}\right]$, and which are the energy density and the tangential pressure for a shell matching Minkowski to the Schwarzschild spacetime. 
IV. NONEXTREMAL ELECTRIC THIN SHELLS INSIDE THE CAUCHY RADIUS: TENSION SHELL REGULAR AND NONREGULAR BLACK HOLES AND COMPACT SHELL NAKED SINGULARITIES

\section{A. Nonextremal electric thin shells inside the Cauchy horizon: Tension shell regular and nonregular black holes}

Here we study the case of a fundamental electric thin shell in the nonextremal state, i.e., $r_{+}>r_{-}$or $M>Q$, for which the shell's location obeys $R<r_{-}$, and for which the orientation is such that the normal to the shell points towards $r_{-}$. In this case horizons do exist and so, following the nomenclature, $r_{+}$is both the gravitational and the event horizon radius, and $r_{-}$is both the Cauchy radius and the Cauchy horizon radius. The normal to the shell pointing towards $r_{-}$means in the notation of the Kruskal coordinate $X$ that we take $\operatorname{sign}(X)=+1$, see the end of this section and Appendix A for details.

As functions of $M, Q$, and $R$, the shell's energy density $\sigma$ and pressure $p$, are, see the end of this section,

$$
\begin{gathered}
8 \pi \sigma=\frac{2}{R}(1-k), \\
8 \pi p=\frac{1}{2 R k}\left[(1-k)^{2}-\frac{Q^{2}}{R^{2}}\right],
\end{gathered}
$$

respectively, with $k=\sqrt{1-\frac{2 M}{R}+\frac{Q^{2}}{R^{2}}}$. Also, the electric charge density $\sigma_{e}$ is given in terms of $M, Q$, and $R$, by

$$
8 \pi \sigma_{e}=\frac{2 Q}{R^{2}}
$$

The behavior of $\sigma$ and $p$ as functions of the radial coordinate $R$ of the shell for various values of the $\frac{Q}{M}$ ratio in this case is shown in Figure 9. We see that, depending on the radial coordinate of the shell, the energy density might take negative values. Indeed, from Eq. (41) we find that for $R<\frac{Q^{2}}{2 M}$ the energy density, $\sigma$, is negative. Also, this kind of thin shell is always supported by tension, see also Eq. (42). It is a tension shell. This is related to the fact that the Reissner-Nordström singularity at $r=0$ is repulsive. Moreover, we see that both the energy density and the pressure of the shell diverge to negative infinity as the shell gets closer to $R=0$. On the other hand, in the limit of $R \rightarrow r_{-}$ the pressure diverges to negative infinity, but the energy density, $\sigma$, tends to $4 \pi \sigma=\frac{1}{r_{-}}$. When $Q=0$, i.e., $r_{-}=0$, the solution is the vacuum Schwarzschild solution, since as $R<r_{-}$, one has in the limit $R=0$, which is singular. In relation to the energy conditions of the shell we can say that the null, the weak, the dominant, and the strong energy conditions are never verified in this case, see a detailed presentation ahead. 

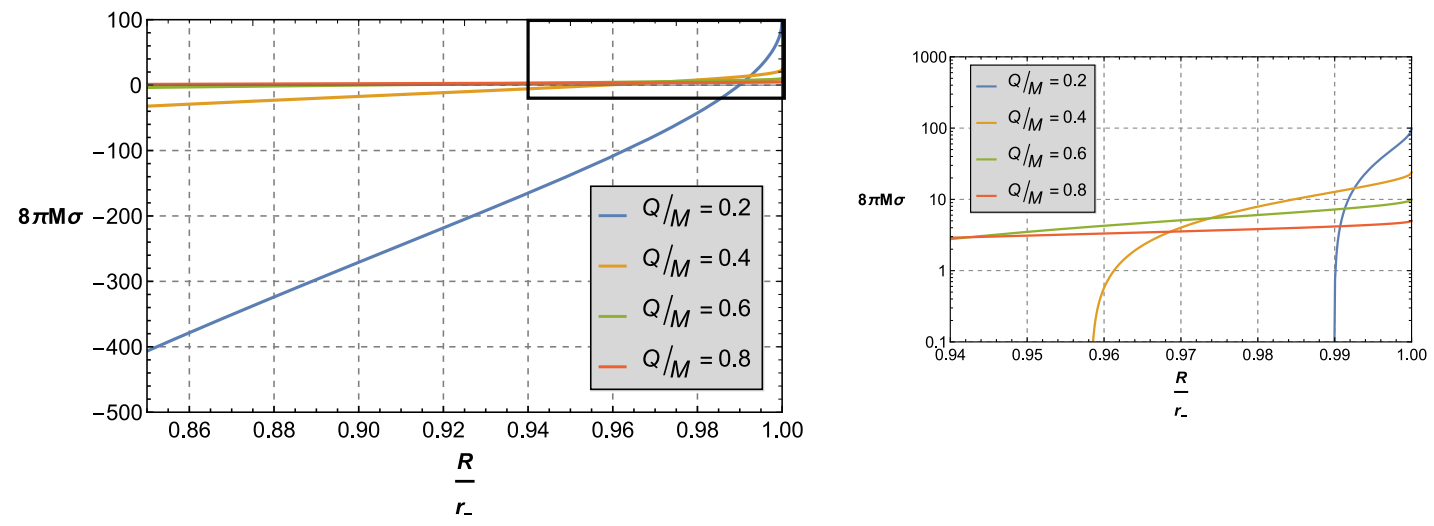

(a)

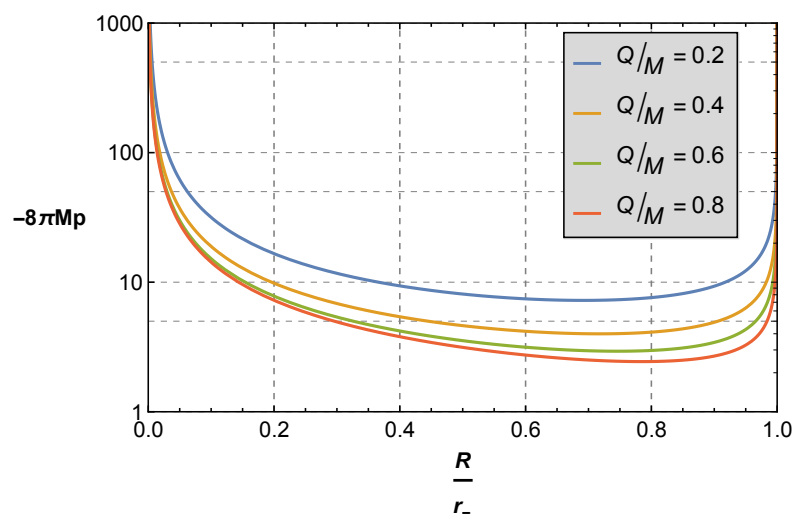

(b)

Figure 9: Physical properties of a nonextremal tension shell regular and nonregular black hole, i.e., an electric perfect fluid thin shell in a nonextremal Reissner-Nordström state, in the location $R<r_{-}$, i.e., located inside the Cauchy radius, and with orientation such that the normal points towards $r_{-}$. The interior is Minkowski and the exterior is nonextremal Reissner-Nordström spacetime. Panel (a) Energy density $\sigma$ of the shell as a function of the radius $R$ of the shell for various values of the $\frac{Q}{M}$ ratio. The energy density is adimensionalized through the mass $M$, $8 \pi M \sigma$, and the radius is adimensionalized through the Cauchy radius $r_{-}, \frac{R}{r_{-}}$. The marked zone on the top left is amplified on the right. Panel (b) Tension $-p$ on the shell as a function of the radius $R$ of the shell for various values of the $\frac{Q}{M}$ ratio. The tension is adimensionalized through the mass $M,-8 \pi M p$, and the radius is adimensionalized through the Cauchy radius $r_{-}, \frac{R}{r_{-}}$.

The Carter-Penrose diagram for this case can be drawn directly from the building blocks of an interior Minkowski spacetime and the full nonextremal Reissner-Nordström spacetime. In Figure 10 two possible Carter-Penrose diagrams of a shell spacetime in a nonextremal Reissner-Nordström state, in the location $R<r_{-}$, with orientation such that the normal points towards $r_{-}$, i.e., $\operatorname{sign}(X)=+1$, are shown. It is a tension shell black hole spacetime. More specifically, there is an infinitude of possible diagrams. Indeed, in the diagram (a) it is clear that the tension shell is inside the Cauchy horizon in both regions III and III' of a Reissner-Nordström spacetime. Admitting that the portion shown of the diagram repeats itself ad infinitum then the black hole is regular. In the diagram (b) there is a shell in region III and a singularity in region III', and so it is not a regular black hole, it is a tension shell black hole with a singularity. Since what one puts in the regions III and III', either a shell or a singularity, is not decided by the solution, an infinite number of different Carter-Penrose diagrams can be drawn, as there are an infinite number of combinations to locate a shell or a singularity when one goes upward or downward through the diagram. Note that $r_{+}$and $r_{-}$are the event horizon and the Cauchy horizon radii, clearly, and the Einstein-Rosen bridge, i.e., the dynamic wormhole, is there. Regular black holes with shells that are sandwiched between a de Sitter interior and a Reissner-Nordström exterior were built in [30]. 


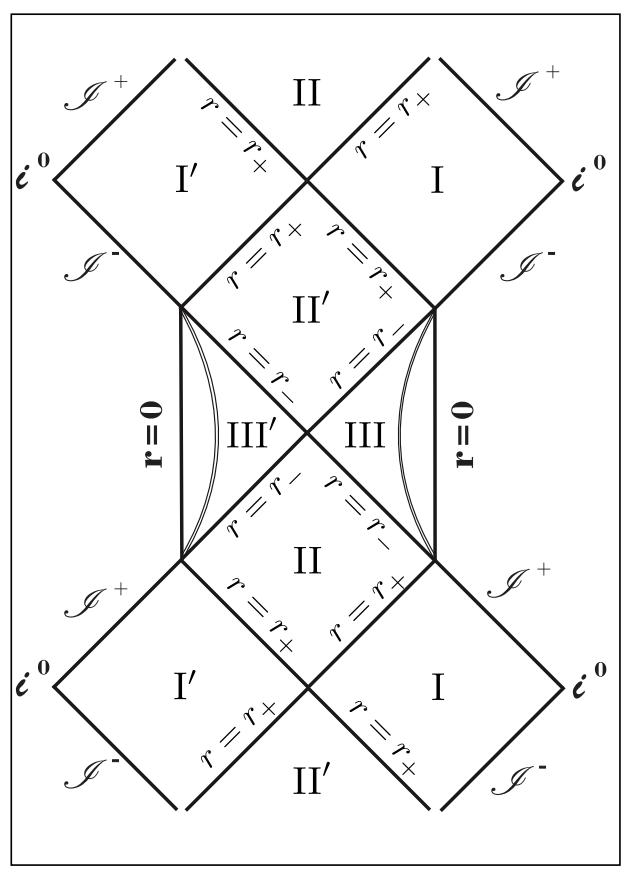

(a)

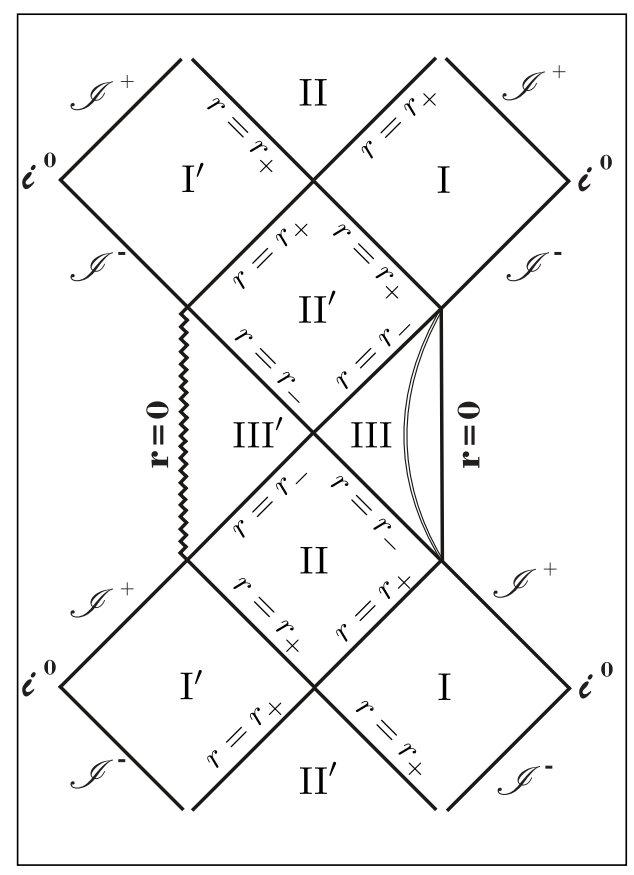

(b)

Figure 10: Carter-Penrose diagrams of the tension shell black holes, i.e., a thin shell spacetime in a nonextremal Reissner-Nordström state, in the location $R<r_{-}$, i.e., located inside the Cauchy radius, with orientation such that the normal to the shell points towards $r_{-}$. The interior is Minkowski, the exterior is Reissner-Nordström spacetime. Panel (a) The Carter-Penrose diagram contains a shell in both regions III and III'. If this pattern is repeated ad infinitum then it is a tension shell regular black hole. Panel (b) The Carter-Penrose diagram contains a shell in region III and a singularity in region III'. It is a tension shell black hole, now not regular. An infinite number of different Carter-Penrose diagrams can be drawn, since there are an infinite number of combinations to locate the shell.

The physical interpretation of this case is of real interest. This nonextremal thin shell solution provides a regular black hole solution. The energy density and pressure never obey the energy conditions for all shell radii, i.e., shell radii between zero and the Cauchy horizon. The causal and global structure as displayed by the Carter-Penrose diagram shows clearly that there is no singularity if one adopts the simplest form of the diagram, meaning also that the topology of the region inside the Cauchy horizons is a three-sphere, as usual for regular black holes. As in the Reissner-Nosdström vacuum solution, this tension shell regular black holes possess Cauchy horizons, and so they are subject to instabilities, which would lead the solutions to an endpoint which can only be guessed. As regular black holes these solutions join the other known regular black hole solutions which are of interest in quantum gravitational settings that presumably get rid of the singularities. So, this case falls into the category of having the energy conditions never verified, and so in this sense is odd, although of interest as regular black hole matter solutions always are. As much as a regular black hole is familiar so this shell solution is familiar. 


\section{B. Nonextremal electric thin shells inside the Cauchy radius: Compact shell naked singularities}

Here we study the case of a fundamental electric thin shell in the nonextremal state, i.e., $r_{+}>r_{-}$or $M>Q$, for which the shell's location obeys $R<r_{-}$, and for which the orientation is such that the normal to the shell points towards $r=0$. In this case, horizons do not exist and so, following the nomenclature, $r_{+}$is the gravitational radius, and $r_{-}$is the Cauchy radius. The normal to the shell pointing towards $r=0$ means in the notation of the Kruskal coordinate $X$ that we take $\operatorname{sign}(X)=-1$, see the end of this section and Appendix A for details.

As functions of $M, Q$, and $R$, the shell's energy density $\sigma$ and pressure $p$, are, see the end of this section,

$$
\begin{gathered}
8 \pi \sigma=\frac{2}{R}(1+k), \\
8 \pi p=-\frac{1}{2 R k}\left[(1+k)^{2}-\frac{Q^{2}}{R^{2}}\right],
\end{gathered}
$$

respectively, with $k=\sqrt{1-\frac{2 M}{R}+\frac{Q^{2}}{R^{2}}}$. The electric charge density $\sigma_{e}$ is given in terms of $M, Q$, and $R$ by Eq. (43). The behavior of $\sigma$ and $p$ as functions of the radial coordinate $R$ of the shell for various values of the $\frac{Q}{M}$ ratio in this case is shown in Figure 11. We see that the energy density of the shell is always positive and the shell is supported

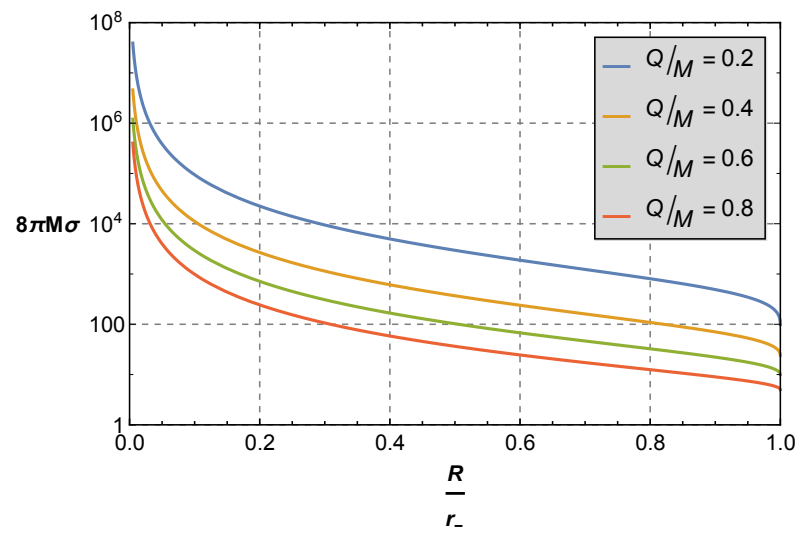

(a)

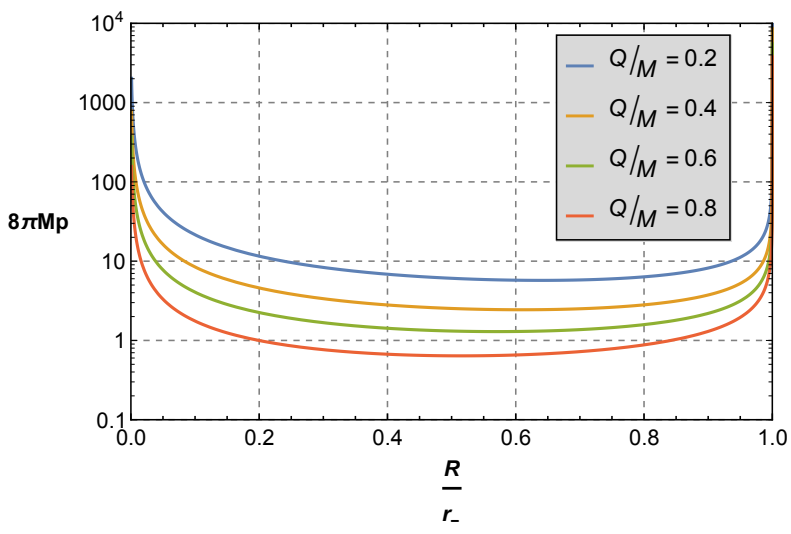

(b)

Figure 11: Physical properties of a nonextremal compact thin shell singularity, i.e., an electric perfect fluid thin shell in a nonextremal Reissner-Nordström state, in the location $R<r_{-}$, i.e., located inside the Cauchy radius, and with orientation such that the normal points towards $r=0$. The interior is Minkowski and the exterior is nonextremal Reissner-Nordström spacetime, although what it is interior and what is exterior is blurred in this case. Panel (a) Energy density $\sigma$ of the shell as a function of the radius $R$ of the shell for various values of the $\frac{Q}{M}$ ratio. The energy density is adimensionalized through the mass $M, 8 \pi M \sigma$, and the radius is adimensionalized through the Cauchy radius $r_{-}, \frac{R}{r_{-}}$. Panel (b) Pressure $p$ on the shell as a function of the radius $R$ of the shell for various values of the $\frac{Q}{M}$ ratio. The pressure is adimensionalized through the mass $M, 8 \pi M p$, and the radius is adimensionalizedthrough the Cauchy radius $r_{-}, \frac{R}{r_{-}}$.

by pressure. As the radial coordinate of the shell, $R$, goes to zero, both the energy density and pressure of the shell diverge to infinity. Moreover, as $R \rightarrow r_{-}$the energy density tends to $\frac{1}{4 \pi r_{-}}$and the pressure diverges to infinity. When $Q=0$ the solution does not exist. In relation to the energy conditions of the shell we can say that the null and the weak energy conditions are verified for $0<R<r_{-}$, the dominant energy condition is verified for $0<R<R_{\mathrm{III}}$, with $R_{\mathrm{III}}$ to be given later, and the strong energy condition is verified for $0<R<r_{-}$, see a detailed presentation ahead. 
The Carter-Penrose diagram for this case can be drawn directly from the building blocks of an interior Minkowski spacetime and the full nonextremal Reissner-Nordström spacetime. In Figure 12 the Carter-Penrose diagram of a shell spacetime in a nonextremal Reissner-Nordström state, in the location $R<r_{-}$, with orientation such that the normal points towards $r=0$, i.e., $\operatorname{sign}(X)=-1$, is shown. It is a compact shell naked singularity spacetime. It is clearly a compact space, $r$ goes from 0 to $R$ and then decreases back to 0 at the timelike singularity, such that there is no clear distinction what is interior from what is exterior. We use the hash symbol \# to represent the connected sum of the spacetime manifolds, in order to conserve the conformal structure in the Carter-Penrose diagram of the total spacetime. It is difficult to understand if this solution can be achieved from a physical phenomenon. However, we expect the shell to be the source of the singularity since the shell is the source of the exterior spacetime, although it is very difficult to understand why the singularity is formed away from the shell itself. Nonetheless, surely the non-linearity of the theory just leads to this counterintuitive behavior.

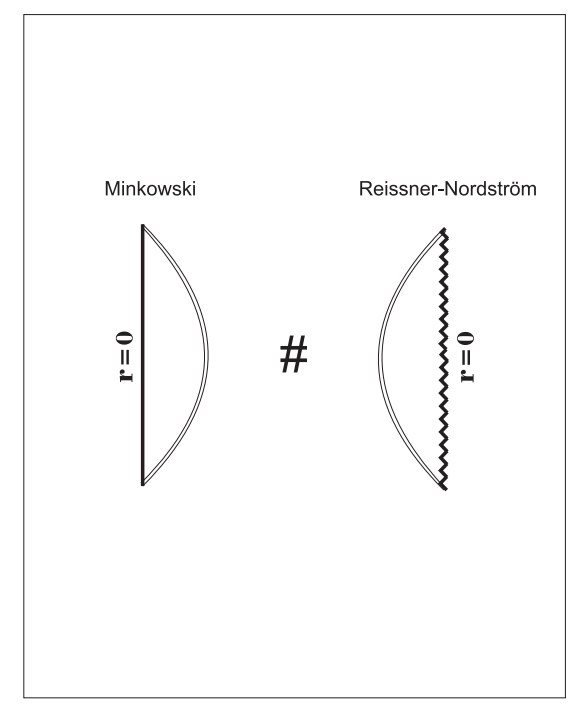

Figure 12: Carter-Penrose diagram of the compact shell naked singularity, i.e., a thin shell spacetime in a nonextremal Reissner-Nordström state, in the location $R<r_{-}$, i.e., located inside the Cauchy radius, with orientation such that the normal to the shell points towards $r=0$. Part of the spacetime is Minkowski, part is Reissner-Nordström, in this case there is no clear distinction what is interior from what is exterior. The hash symbol \# represents the connected sum of the two spacetimes.

The physical interpretation of this case is most curious. This nonextremal thin shell solution provides a closed spatial static universe with a singularity at one pole. The energy density and pressure obey the energy conditions for certain shell radii. The causal and global structure as displayed by the Carter-Penrose diagram show the characteristics of this universe that has two sheets joined at the shell with one sheet having a singularity at its pole and with no horizons. So, this case falls into the category of having the energy conditions verified and the resulting spacetime being peculiar. 


\section{Formalism for nonextremal electric thin shells inside the Cauchy radius}

\section{Preliminaries}

We now make a careful study to derive the properties of the fundamental electric thin shell used in the two previous subsections, i.e., the thin shell in the nonextremal state, i.e., $r_{+}>r_{-}$or $M>Q$, for which the shell's location obeys $R<r_{-}$, and for which the orientation is such that the normal to the shell points towards $r_{-}$or towards $r=0$. It should be read as an appendix to the previous two subsections. We use the formalism developed in Sec. II and Appendix A.

\section{Induced metric and extrinsic curvature of $\mathcal{S}$ as seen from $\mathcal{M}_{\mathrm{i}}$}

Let us start by mentioning the interior Minkowski spacetime, $\mathcal{M}_{i}$. Since it is the same as the analysis done in the previous section we only quote the important equations. They are the interior metric Eq. (28), the interior fourvelocity of the shell Eq. (29), the metric for the shell at radius $R$ given in Eq. (30), the normal to the shell Eq. (31), and the extrinsic curvature from the inside Eq. (32).

\section{Induced metric, and extrinsic curvature of $\mathcal{S}$ as seen from $\mathcal{M}_{\mathrm{e}}$}

To proceed we have now to find the expressions for the induced metric on $\mathcal{S}$ and the extrinsic curvature components as seen from the exterior spacetime, $\mathcal{M}_{\mathrm{e}}$, in the nonextremal state, i.e., $r_{+}>r_{-}$or $M>Q$, see Figure 2 , for which the shell's location has radius $R$ obeying $R<r_{-}$, and for which the orientation is such that the normal to the shell points towards increasing $r$, i.e., towards $r_{-}$, or towards decreasing $r$, i.e., towards $r=0$, as seen from the exterior, as used in the two previous subsections.

For a nonextremal shell with $R<r_{-}$we work with the coordinate patch that has no coordinate singularity at the gravitational radius $r=r_{-}$. Many of the previous results are also valid for the second coordinate patch. From the discussion in Appendix A 2, the line element for the Reissner-Nordström spacetime in Kruskal-Szekeres coordinates in this patch is,

$$
\begin{gathered}
d s_{\mathrm{e}}^{2}=4\left(\frac{r_{+}+r_{-}}{r_{+}-r_{-}}\right)^{2} \frac{r_{-}^{4}}{r^{2}} e^{\frac{r\left(r_{+}-r_{-}\right)}{r_{-}^{2}}}\left(\frac{r_{+}-r}{r_{+}+r_{-}}\right)^{1+\left(\frac{r_{+}}{r_{-}}\right)^{2}}\left(d X^{2}-d T^{2}\right)+r^{2}(T, X) d \Omega^{2}, \\
X^{2}-T^{2}=e^{-\frac{r\left(r_{+}-r_{-}\right)}{r_{-}^{2}}}\left(\frac{r_{-}-r}{r_{+}+r_{-}}\right)\left(\frac{r_{+}-r}{r_{+}+r_{-}}\right)^{-\left(\frac{r_{+}}{r_{-}}\right)^{2}}
\end{gathered}
$$

with $r(T, X)$ being given implicitly by the latter equation.

The shell's radial coordinate when measured by an observer at $\mathcal{M}_{\mathrm{e}}$ is constant since the shell is static, so from the second of the equations in Eq. (46) we take that the $X$ and $T$ coordinates of the shell must verify $X^{2}-T^{2}=$ constant. Now, as was argued in the previous section, a static shell must be timelike as seen from both interior and exterior spacetimes. The restriction $X^{2}-T^{2}=$ constant and the analysis performed in subsection IIIC 3 , imply that the components of the 4 -velocity $u_{\mathrm{e}}$ of an observer comoving with the shell as seen from the exterior spacetime, are given by

$$
u_{\mathrm{e}}^{\alpha}=-\sqrt{\frac{g^{X X}}{X^{2}-T^{2}}}(X, T, 0,0),
$$

where, in this case, $g^{X X}$ is the $X X$ component of the inverse of the metric in Eq. (46). We see that Eq. (47) only makes sense physically, if $X^{2}-T^{2}>0$, which, taking into account the second of the equations in Eq. (46), allows us to conclude that the shell must then be located either at the region III or III', see Figure 2. Let us remark that the minus sign in Eq. (47) arises from the convention that the 4-velocity points to the future for both regions III and III'. Making use of Eqs. (46) and (47) to find the induced metric on $\mathcal{S}$ as seen by an observer at $\mathcal{M}_{\mathrm{e}}$ and imposing the first junction condition, Eq. (8), we deduce that the shell's radial coordinate $R$ is the same as measured by an observer at $\mathcal{M}_{\mathrm{i}}$ or $\mathcal{M}_{\mathrm{e}}$ and the induced metric on $\mathcal{S}$ is given by Eq. (35), namely,

$$
\left.d s^{2}\right|_{\mathcal{S}}=-d \tau^{2}+R^{2} d \Omega^{2} .
$$


Combining $n_{\mathrm{e}}^{\alpha} n_{\mathrm{e} \alpha}=1$, see Eq. (3), $n_{\mathrm{e} \alpha} u_{\mathrm{e}}^{\alpha}=0$, see Eq. (4), and Eq. (47), we find the expression for the components of the unit normal to the hypersurface $\mathcal{S}$, as seen from the exterior spacetime $\mathcal{M}_{\mathrm{e}}$, to be $n_{\mathrm{e} \alpha}= \pm \sqrt{\frac{g_{X X}}{X^{2}-T^{2}}}(-T, X, 0,0)$. To specify the sign of the normal to $\mathcal{S}$ for each region we consider two orientations: the orientation where the normal $n_{\mathrm{e} \alpha}$ points towards the Cauchy radius at $r_{-}$and the orientation where the normal points towards the singularity $r=0$. These two orientations can be treated in a concise way by assuming, for example, a shell located either in the region III or III' and the normal pointing in the direction of decreasing $X$ coordinate, such that

$$
n_{\mathrm{e} \alpha}=\operatorname{sign}(X) \sqrt{\frac{g_{X X}}{X^{2}-T^{2}}}(T,-X, 0,0) .
$$

Note the importance of the sign of the normal to yield totally different physical and geometrical properties to a shell in the same location, here in the region $R<r_{-}$. Then, using the results from Appendix B 2, we find the nonzero components of the extrinsic curvature of $\mathcal{S}$ as seen from the exterior spacetime to be given by

$$
K_{\mathrm{e}}{ }^{\tau} \tau=\frac{\operatorname{sign}(X)}{2 R^{2} k}\left[r_{+}+r_{-}-2 \frac{r_{+} r_{-}}{R}\right], \quad K_{\mathrm{e}}{ }^{\theta}{ }_{\theta}=K_{\mathrm{e}}{ }^{\varphi} \varphi=\frac{\operatorname{sign}(X)\left(r_{+}-r_{-}\right)}{2 r_{-}^{2} R} \sqrt{g_{X X}\left(X^{2}-T^{2}\right)},
$$

where $k$, here, is the redshift function given in Eq. (20), evaluated at $R$, i.e., $k\left(R, r_{+}, r_{-}\right)=\sqrt{\left(1-\frac{r_{+}}{R}\right)\left(1-\frac{r_{-}}{R}\right)}$.

A comment is in order here. In our study of a shell in a nonextremal Reissner-Nordström state, we have worked with two coordinate patches to describe the various regions of the Reissner-Nordström spacetime exterior to the shell as was done in [13], see also Appendix A. It is possible to find a coordinate system that covers the entire Reissner-Nordström spacetime without coordinate singularities, see [38] and also [40] or [41-43], but we have not followed this path, as it is not the best one to our aims, and thus we have separated the study of a shell located in a region described by one coordinate patch and the other.

\section{Shell's energy density and pressure}

We are now in position to find the properties of a perfect fluid thin shell in a nonextremal Reissner-Nordström state, located inside the Cauchy horizon radius or Cauchy radius, depending on the case. The shell's stress-energy tensor is given in Eq. (10), an expression containing the energy per unit area $\sigma$, the tangential pressure of the fluid $p$, the four-velocity $u_{a}$, and the induced metric $h_{a b}$. From our choice of coordinates on $\mathcal{S}$ we have that $\left\{y^{a}\right\}=(\tau, \theta, \varphi)$, the four-velocity $u_{a}$ is given in Eq. (47), and the metric $h_{a b}$ is given in Eq. (48). Putting everything together we find $S_{\tau}^{\tau}=-\sigma, S_{\theta}^{\theta}=S_{\varphi}^{\varphi}=p$. Comparing these latter equations with the second junction condition, Eq. (9), taking into account the components of the induced metric, given through Eq. (48), and the fact that $\left[K_{\theta}^{\theta}\right]=\left[K_{\varphi}^{\varphi}\right]$, we find $\sigma=-\frac{1}{4 \pi}\left[K_{\theta}^{\theta}\right]$ and $p=\frac{1}{8 \pi}\left[K_{\tau}^{\tau}\right]-\frac{\sigma}{2}$. With the components of the extrinsic curvature found in Eqs. (32) and (50) we obtain the following properties of a perfect fluid thin shell located inside of the Cauchy radius,

$$
\begin{gathered}
8 \pi \sigma=\frac{2}{R}(1-\operatorname{sign}(X) k), \\
8 \pi p=\frac{\operatorname{sign}(X)}{2 R k}\left[(1-\operatorname{sign}(X) k)^{2}-\frac{r_{+} r_{-}}{R^{2}}\right],
\end{gathered}
$$

where $k$ here is the redshift function given in Eq. (20) evaluated at $R$, i.e., $k\left(R, r_{+}, r_{-}\right)=\sqrt{\left(1-\frac{r_{+}}{R}\right)\left(1-\frac{r_{-}}{R}\right)}$. As the surface electric current density $s_{a}$ on the thin shell is defined as $s_{a}=\sigma_{e} u_{a}$, where $\sigma_{e}$ represents the electric charge density and $u_{a}$ is the velocity of the shell, from Eqs. (12)-(13) and (21) it follows that

$$
8 \pi \sigma_{e}=2 \frac{\sqrt{r_{+} r_{-}}}{R^{2}}
$$

Now, the expressions found for the energy density and pressure for a shell locate inside the Cauchy radius, Eqs. (51) and (52), are the same as Eqs. (38) and (39) found for for the energy density and pressure for a shell locate outside the gravitational radius. However, the behavior of the properties of the shell will be different since, the radial coordinate of the shell, $R$, in this case ranges between zero and $r_{-}$. As before, we have to distinguish the two possible orientations provided by the sign $(X)$. In Eqs. (51) and (52) it is clear that it is necessary to pick the sign in sign $(X)$. Let us start with $\operatorname{sign}(X)=+1$. It is useful to give the expressions for the shell's energy density and pressure, $\sigma$ and 
$p$ in terms of $M$ and $Q$. Using Eq. (22) in Eqs. (38) and (39) with $\operatorname{sign}(X)=+1$ we have $8 \pi \sigma=\frac{2}{R}(1-k)$, $8 \pi p=\frac{1}{2 R k}\left[(1-k)^{2}-\frac{Q^{2}}{R^{2}}\right]$, and also from Eq. (40) we have $8 \pi \sigma_{e}=\frac{2 Q}{R^{2}}$. Let us now take $\operatorname{sign}(X)=-1$. It is useful to give the expressions for the shell's energy density and pressure, $\sigma$ and $p$ in terms of $M$ and $Q$. Using Eq. (22) in Eqs. (38) and (39) with $\operatorname{sign}(X)=-1$ we have $8 \pi \sigma=\frac{2}{R}(1+k), 8 \pi p=-\frac{1}{2 R k}\left[(1+k)^{2}-\frac{Q^{2}}{R^{2}}\right]$, with $k=\sqrt{1-\frac{2 M}{R}+\frac{Q^{2}}{R^{2}}}$, and also from Eq. (40) we have $8 \pi \sigma_{e}=\frac{2 Q}{R^{2}}$. These are the expressions used in the two previous subsections. Note also that when $r_{-}=0$, then $\sigma_{e}=0$ and the electric charge $Q$ is zero, $Q=0$, and since $R<r_{-}$we obtain that there either the solution is vacuum and singular or there is no solution, in brief, there is no shell solution. 


\section{EXTREMAL ELECTRIC THIN SHELLS OUTSIDE THE GRAVITATIONAL RADIUS: MAJUMDAR-PAPAPETROU STAR SHELLS AND EXTREMAL TENSION SHELL SINGULARITIES}

\section{A. Extremal electric thin shells outside the gravitational radius: Majumdar-Papapetrou star shells}

Here we study the case of a fundamental electric thin shell in the extremal state, i.e., $r_{+}=r_{-}$or $M=Q$, and indeed, $r_{+}=r_{-}=M=Q$, for which the shell's location obeys $R>r_{+}$, and for which the orientation is such that the normal to the shell points towards spatial infinity. In this case horizons do not exist and so, following the nomenclature, $r_{+}$is the gravitational radius. Also, since $r_{+}$and $r_{-}$have the same value we opt to use consistently the gravitational radius $r_{+}$rather than the Cauchy radius $r_{-}$. In general we also opt to use $M$ rather than $Q$. The normal to the shell pointing towards spatial infinity means that the new parameter $\xi$ we introduce for the extremal states has value $\xi=+1$, see the end of this section.

As functions of $M$ and $R$, the shell's energy density $\sigma$ and pressure $p$, are, see the end of this section,

$$
\begin{aligned}
& 8 \pi \sigma=\frac{2 M}{R^{2}}, \\
& 8 \pi p=0 .
\end{aligned}
$$

Also, the electric charge density $\sigma_{e}$ is given in terms of $M$ and $R$, by

$$
8 \pi \sigma_{e}=\frac{2 M}{R^{2}}
$$

The behavior of $\sigma$ and $p$, in Eqs. (54) and (55), as functions of the radial coordinate $R$ of the $\frac{Q}{M}=1$ extremal shell is shown in Figure 13. These shells are characterized by a positive energy density and vanishing pressure support, and

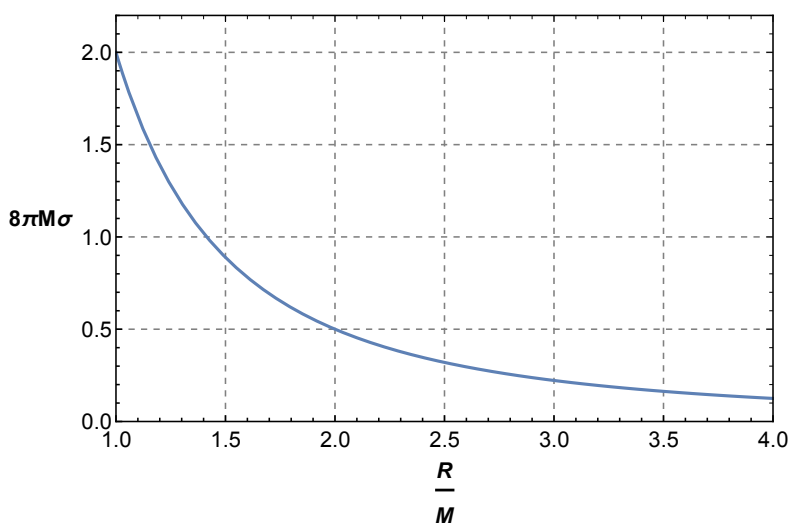

(a)

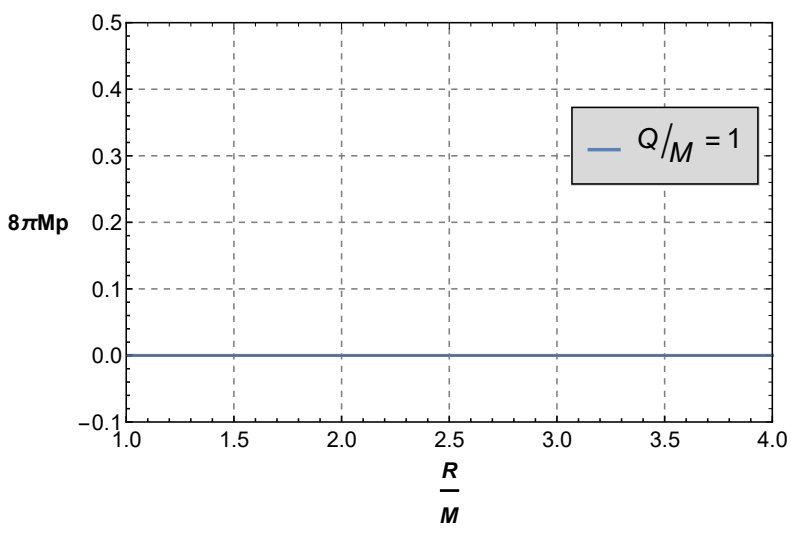

(b)

Figure 13: Physical properties of a Majumdar-Papapetrou star shell, i.e., an electric perfect fluid thin shell in an extremal Reissner-Nordström state, in the location $R>r_{+}$, i.e., located outside the gravitational radius, and with

orientation such that the normal points towards spatial infinity. The interior is Minkowski and the exterior is extremal Reissner-Nordström spacetime. Extremal means $\frac{Q}{M}=1$. Panel (a) Energy density $\sigma$ of the shell as a function of the radius $R$ of the shell. The energy density is adimensionalized through the mass $M, 8 \pi M \sigma$, and the radius is adimensionalized through the gravitational radius $r_{+}, \frac{R}{r_{+}}$. Panel (b) Pressure $p$ on the shell as a function of the radius $R$ of the shell. The radius is adimensionalized through the gravitational radius $r_{+}, \frac{R}{r_{+}}$. The pressure is zero, the shell is supported by electric repulsion alone, it is Majumdar-Papapetrou matter.

so the matter that composes this kind of shells is Majumdar-Papapetrou matter, i.e., electric dust, there is no need for matter pressure since there is an inbuilt equilibrium between gravitational attraction and electrostatic repulsion. These are extremal star shells or Majumdar-Papapetrou star shells. Majumdar-Papapetrou matter shells with a Minkowski interior matched to an exterior extremal Reissner-Nordström spacetime, with the implicit assumption that the outward unit normal to the matching surface points towards spacial infinity, have been considered in many works. Notice that when $R \rightarrow \infty$, the energy density $\sigma$ and the charge density $\sigma_{e}$, all tend to zero, i.e., the shell disperses away. Notice also that when $R \rightarrow r_{+}$, the energy density is finite, the pressure remains zero, and the charge density 
$\sigma_{e}$ is also finite. Indeed, for $R=r_{+}$one has a quasiblack hole, discussed in detail ahead. When $Q=0$, and so $M=0$, there is no shell, only Minkowski spacetime. In relation to the energy conditions of the shell one can work out and find that the null, the weak, the dominant, and the strong energy conditions are verified for $R>r_{+}$, see a detailed presentation ahead.

The Carter-Penrose diagram can be drawn directly from the building blocks of an interior Minkowski spacetime and the exterior asymptotic region of an extremal Reissner-Nordström spacetime. In Figure 14 the Carter-Penrose diagram of an extremal Reissner-Nordström shell spacetime for a junction surface with normal pointing towards spatial infinity is shown. It is clearly a star shell, a Majumdar-Papapetrou star shell in an asymptotically flat spacetime.

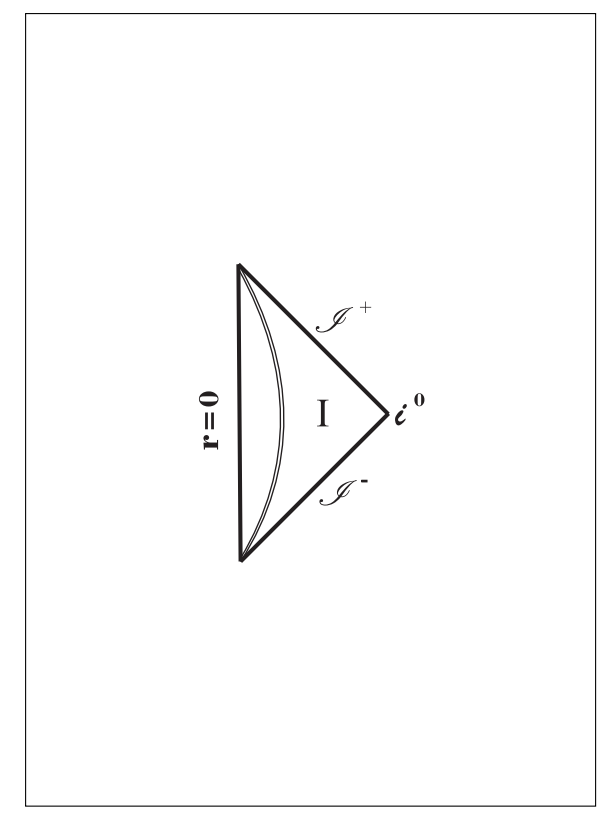

Figure 14: Carter-Penrose diagram of a Majumdar-Papapetrou star shell, i.e., a thin shell spacetime in an extremal Reissner-Nordström state, located at $R>r_{+}$, i.e., located outside the gravitational radius, with orientation such that the normal points towards spatial infinity. The interior is Minkowski, the exterior is extremal Reissner-Nordström. This star shell is supported by electrical repulsion alone.

The physical interpretation of this case is clear cut, and it is similar to the corresponding nonextremal shell. This extremal thin shell solution mimics an extremal star. The energy density and pressure obey the energy conditions for any radius, indeed the shell is composed of Majumdar-Papapetrou matter. The causal and global structure as displayed by the Carter-Penrose diagram are well behaved and rather elementary. So, this case falls into the category of having the energy conditions verified and the geometrical setup is physically reasonable. 


\section{B. Extremal electric thin shells outside the event horizon: Extremal tension shell singularities}

Here we study the case of a fundamental electric thin shell in the extremal state, i.e., $r_{+}=r_{-}$or $M=Q$, and indeed, $r_{+}=r_{-}=M=Q$, for which the shell's location obeys $R>r_{+}$, and for which the orientation is such that the normal to the shell points towards $r_{+}$. In this case horizons do exist and so, following the nomenclature, $r_{+}$is both the gravitational and the event horizon radius. Also, $r_{+}$and $r_{-}$have the same value and we opt to use the event horizon radius $r_{+}$rather than the Cauchy horizon radius $r_{-}$. We also opt to use $M$ rather than $Q$. The normal to the shell pointing towards $r_{+}$means in the notation we use that we take $\xi=-1$, see the end of this section for details.

As functions of $M$ and $R$, the shell's energy density $\sigma$ and pressure $p$, are, see the end of this section,

$$
\begin{aligned}
& 8 \pi \sigma=\frac{2}{R}\left(2-\frac{M}{R}\right), \\
& 8 \pi p=-\frac{2}{R} .
\end{aligned}
$$

The electric charge density $\sigma_{e}$ is given in terms of $M$ and $R$ by $8 \pi \sigma_{e}=\frac{2 M}{R^{2}}$, which is identical to Eq. (56). The behavior of $\sigma$ and $p$, in Eqs. (57) and (58), as functions of the radial coordinate $R$ of the $\frac{Q}{M}=1$ extremal shell is shown in Figure 15. The matter fluid that composes such shells is characterized by positive energy density $\sigma$ and

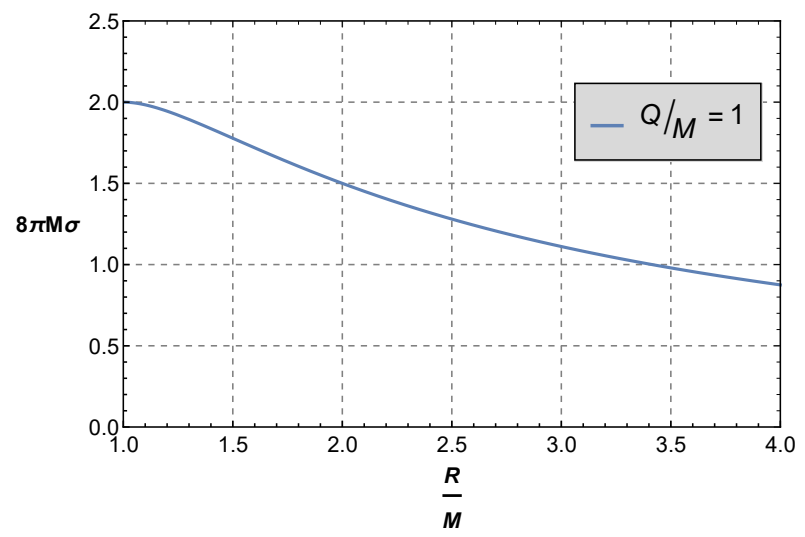

(a)

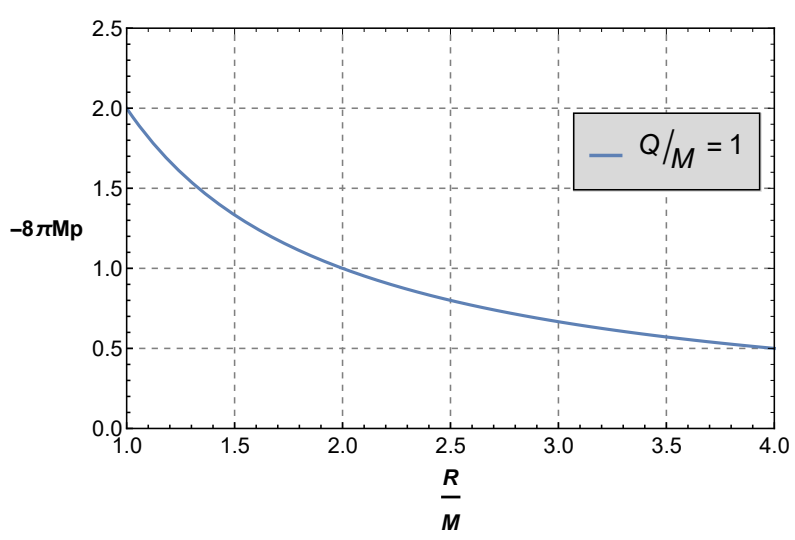

(b)

Figure 15: Physical properties of an extremal tension shell singularity, i.e., an electric perfect fluid thin shell in an extremal Reissner-Nordström state, in the location $R>r_{+}$, i.e., located outside the event horizon, with orientation such that the normal points towards $r_{+}$. The interior is Minkowski, the exterior is extremal Reissner-Nordström spacetime. Extremal means $\frac{Q}{M}=1$. Panel (a) Energy density $\sigma$ of the shell as a function of the radius $R$ of the shell. The energy density is adimensionalized through the mass $M, 8 \pi M \sigma$, and the radius is adimensionalized through the gravitational radius $r_{+}, \frac{R}{r_{+}}$. Panel (b) Pressure $p$ on the shell as a function of the radius $R$ of the shell.

The pressure is negative, so the shell is supported by tension. The radius is adimensionalized through the gravitational radius $r_{+}, \frac{R}{r_{+}}$.

is supported by tension $-p$, with both falling to zero when $R=\infty$. In this case since $p$ is not zero, the shell is not composed of Majumdar-Papapetrou matter. Notwithstanding the exterior spacetime is extremal. Examples of spacetimes for which $M=Q$ globally whose interior is not made of Majumdar-Papapetrou matter, as is the case here, are many. However, this case is of of particular interest since matter properties provided by Eqs. (57)-(58) and the electric charge density $8 \pi \sigma_{e}=\frac{2 M}{R^{2}}$, have specific relevant features. Indeed, $\sigma$ has two terms, namely, an intrinsic geometrical one given by $\frac{4}{R}$ and a gravitational one which is negative given by $-\frac{2 M}{R^{2}}$. These two terms can be considered independent and $\sigma$ is the sum of the two. The first term of $\sigma, \frac{4}{R}$, is a geometrical term that also gives rise to a geometrical tension given by $-\frac{2}{R}$ and ensures that there is a shell for sure caused from the embedding of the shell in the interior and exterior spacetimes, as the radial distance grows up to a maximum at the shell with radius $R$ nd then diminishes to $r_{+}$and finally to zero at the timelike singularity. This geometric term exists independently on whether there is spacetime mass $M$ or not, indeed, the spacetime mass energy coming from this geometrical term is zero since $\frac{4}{R}+2 p=0$. The second term $-\frac{2 M}{R^{2}}$ is negative and can be explained by the fact that due to the electric charge density $8 \pi \sigma_{e}=\frac{2 M}{R^{2}}$ on the shell, there is electric repulsion, and on the other hand, since positive gravity is 
on the direction of $r_{+}$and $r=0$, to counterbalance the electric repulsion and the direction of positive gravity, the shell has to have an anti repulsive negative energy density, an anti gravity term or anti Majumdar-Papapetrou energy density term, of value $-\frac{2 M}{R^{2}}$. Note also that $\sigma+2 p+\sigma_{e}=0$. When $Q=0$, and so $M=0$, there is still a shell of radius $R$, but with a Minkowski spacetime on each side of it. In relation to the energy conditions of the shell one can work out and find that the null, the weak, and the dominant energy conditions are verified for $R>r_{+}$, and the strong energy condition is never verified, see a detailed presentation ahead.

The Carter-Penrose diagram for this case can be drawn directly from the building blocks of an interior Minkowski spacetime and the full extremal Reissner-Nordström spacetime. In Figure 16 the Carter-Penrose diagram of a shell spacetime in an extremal Reissner-Nordström state, in the location $R>r_{+}$, with orientation such that the normal points towards $r_{+}$, i.e., $\xi=-1$, is shown. It has a horizon, but the existence of the singularity is more striking, i.e., it is an extremal tension shell singularity. There is an infinitude of possible diagrams as the maximal analytical

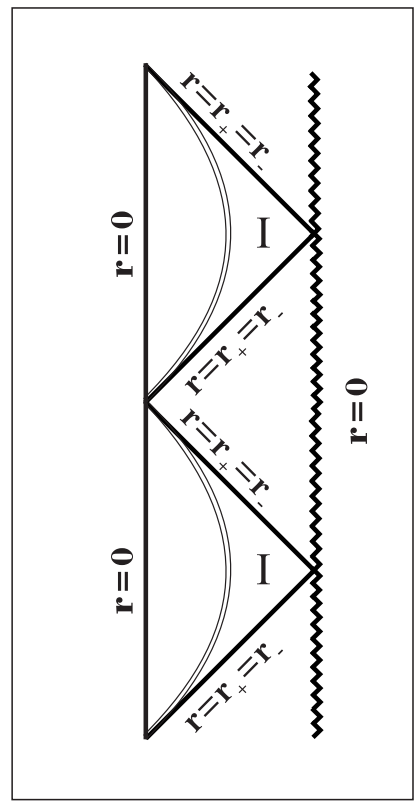

(a)

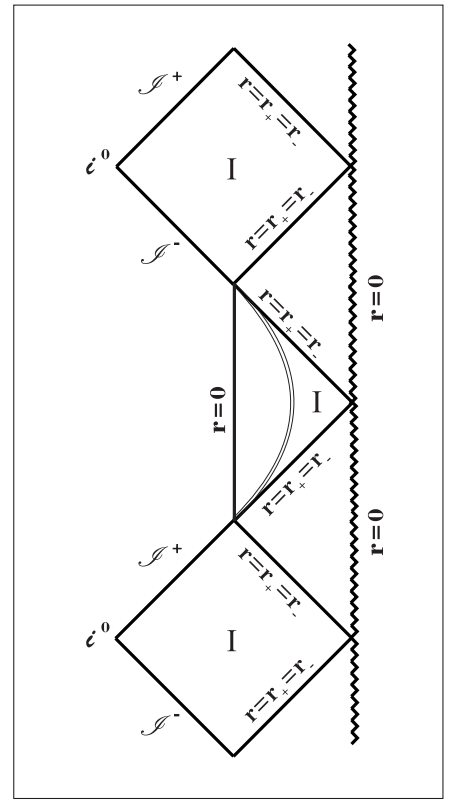

(b)

Figure 16: Carter-Penrose diagrams of an extremal tension shell singularity, i.e., a thin shell spacetime in an extremal Reissner-Nordström state, in the location $R>r_{+}$, i.e., located outside the event horizon, with orientation such that the normal points towards $r_{+}$. The interior is Minkowski, the exterior is extremal Reissner-Nordström spacetime. Panel (a) The Carter-Penrose diagram contains a shell in region I and repeats itself upwards. Panel (b) The Carter-Penrose diagram contains a shell in the regions I shown that passes into asymptotically flat regions. An infinite number of different Carter-Penrose diagrams can be drawn, since there are an infinite number of combinations to place the shell and infinity.

extension of the resulting spacetime can always contain a thin matter shell outside the event horizon or only at a discrete number of these regions. In the diagram (a) the tension shell is outside the event horizon in region I. Then, the tension shell repeats itself in the next portion of the diagram. It is a compact tension shell that repeats itself. In the diagram (b) of the figure the tension shell is outside the event horizon in region I. Then, an asymptotic infinity takes over in the next portion of the diagram. Since what one puts in the regions I, either a shell or infinity, is not decided by the solution, indeed an infinite number of different Carter-Penrose diagrams can be drawn, as there are an infinite number of combinations to locate a shell or infinity when one goes upward or downward through the diagram. This is a tension shell, but since it is extremal there is no Einstein-Rosen bridge, no dynamic wormhole.

The physical interpretation of this case is somewhat simple, with the case itself being unusual. This extremal thin shell solution, in its simplest form, turns the space around up to a horizon and then opens up to another universe with another shell, or to a singularity, and so on. The energy density and pressure have special features as has been just pointed out, and obey some of the energy conditions. The causal and global structures as displayed by the Carter-Penrose diagram show the unique features of this spacetime. So, this case falls into the category of having some of the energy conditions verified and the geometrical setup is strange. 


\section{Formalism for extremal electric thin shells outside the gravitational radius}

\section{Preliminaries}

We now make a careful study to derive the properties of the fundamental electric thin shell used in the two previous subsections, i.e., the thin shell in an extremal state, i.e., $r_{+}=r_{-}$or $M=Q$, and indeed $r_{+}=r_{-}=M=Q$, for which the shell's radius $R$ location obeys $R>r_{+}$, and for which the orientation is such that the normal to the shell points towards infinity or towards $r_{+}$. It should be read as an appendix to the previous two subsections. We use the formalism developed in Sec. II.

\section{Induced metric, and extrinsic curvature of $\mathcal{S}$ as seen from $\mathcal{M}_{\mathrm{i}}$}

Let us start by analyzing the interior Minkowski spacetime, $\mathcal{M}_{\mathrm{i}}$. Since it is the same as the analysis done previously we only quote the important equations. They are the interior metric Eq. (28), the interior four-velocity of the shell Eq. (29), the metric for the shell at radius $R$ Eq. (30), the normal to the shell Eq. (31), and the extrinsic curvature from the inside Eq. (32).

\section{Induced metric, and extrinsic curvature of $\mathcal{S}$ as seen from $\mathcal{M}_{\mathrm{e}}$}

To proceed we have now to find the expressions for the induced metric on $\mathcal{S}$ and the extrinsic curvature components as seen from the exterior spacetime, $\mathcal{M}_{\mathrm{e}}$, in the extremal state, i.e., $r_{+}=r_{-}$or $M=Q$, see Figure 3 , for which the shell's radius $R$ location obeys $R>r_{+}$, and for which the orientation is such that the normal to the shell points towards increasing $r$, i.e., towards infinity, or towards decreasing $r$ i.e., towards $r_{+}$, as seen from the exterior, as used in the two previous subsections.

For an extremal shell located at $R>r_{+}$one has also to be concerned about the normal vector to the shell. In the extremal Reissner-Nordström spacetime there is no Einstein-Rosen-bridge and so there is no ambiguity in the definition of the radial coordinate as the value of the circumferential radius. Thus, there is no need for the KruskalSkekeres $(T, X, \theta, \varphi)$ coordinates and we can resort in this analysis of the induced metric and extrinsic curvature of the matching surface using simply the Schwarzschild coordinates $(t, r, \theta, \varphi)$. The Reissner-Nordström line element for the exterior extremal solution is

$$
d s_{\mathrm{e}}^{2}=-\left(1-\frac{r_{+}}{r}\right)^{2} d t^{2}+\frac{d r^{2}}{\left(1-\frac{r_{+}}{r}\right)^{2}}+r^{2} d \Omega^{2} .
$$

Assuming the circumferential radius of the matching surface $\mathcal{S}$ to be described by a function $R(\tau)$, where $\tau$ is the proper time of an observer comoving with $\mathcal{S}$ and imposing the shell to be static implies that $\frac{d R}{d \tau}=0$. Then, the 4 -velocity of an observer comoving with $\mathcal{S}$, as seen from $\mathcal{M}_{\mathrm{e}}$, is given by

$$
u_{\mathrm{e}}^{\alpha}=\left(\frac{1}{k}, 0,0,0\right),
$$

where, in this situation the redshift function $k$ at $\mathcal{S}$ is given in Eq. (20), evaluated at $R$, i.e., $k\left(R, r_{+}=r_{-}\right) \equiv$ $k\left(R, r_{+}\right)=1-\frac{r_{+}}{R}$. Equation (60) can now be used to compute the induced metric on $\mathcal{S}$ by $\mathcal{M}_{\mathrm{e}}$, and we find $\left.d s_{\mathrm{e}}^{2}\right|_{\mathcal{S}}=-d \tau^{2}+R^{2} d \Omega^{2}$. Imposing the the first junction condition Eq. (8) and Eq. (30) we find that the shell's radial functions at each sice of $\mathcal{S}$ are the same, and so the matching surface $\mathcal{S}$ is characterized by the line element

$$
\left.d s^{2}\right|_{\mathcal{S}}=-d \tau^{2}+R^{2} d \Omega^{2} .
$$

Using the normalization and orthogonality relations (3) and (4) allows us to find the following expression for the normal

$$
n_{\mathrm{e} \alpha}=\xi\left(0, \frac{1}{k}, 0,0\right)
$$

where the parameter $\xi=\{-1,1\}$ is defined as $\xi=+1$ if the outside unit normal to the shell points in the direction of increasing radial coordinate $r$, measured by an observer in the exterior $\mathcal{M}_{\mathrm{e}}$ spacetime, and $\xi=-1$ if the outside unit normal to the shell points in the direction of decreasing radial coordinate $r$, again, measured by an observer in the 
exterior $\mathcal{M}_{\mathrm{e}}$ spacetime. In the extremal case the parameter $\xi$ takes the place of the sign $(X)$ used in the nonextremal case. Taking into account Eqs. (4), (60), and (62) we find that the nonzero components of the extrinsic curvature of the matching surface, see Eq. (6), are given by

$$
K_{\mathrm{e}}^{\tau} \tau=\xi \frac{r_{+}}{R^{2}}, \quad K_{\mathrm{e}}{ }^{\theta} \theta=K_{\mathrm{e}}^{\varphi} \varphi=\xi \frac{k}{R}
$$

\section{Shell's energy density and pressure}

Having determined the components of the extrinsic curvature of the matching surface $\mathcal{S}$ as seen from the interior and exterior spacetimes we are now in position to use the second junction condition given in Eq. (9) to find the expressions for the energy density and pressure support of the extremal thin shell in these cases. The shell's stress-energy tensor is given in Eq. (10), so Eqs. (32) and (63) yield

$$
\begin{aligned}
& 8 \pi \sigma=\frac{2}{R}\left[1-\xi\left(1-\frac{r_{+}}{R}\right)\right], \\
& 8 \pi p=\frac{1}{R}(\xi-1),
\end{aligned}
$$

where again here $k=1-\frac{r_{+}}{R}$. Note that $p$ in Eq. (65) is independent of $M$, it only depends on $R$ and thus on the geometry of the shell as embedded in the ambient spacetime. Moreover, since the surface electric current density $s_{a}$ on the thin shell is $s_{a}=\sigma_{e} u_{a}$, where $\sigma_{e}$ represents the electric charge density, and since the Minkowski spacetime has zero electric charge, from Eqs. (12), (13) and (21) it follows that

$$
8 \pi \sigma_{e}=2 \frac{r_{+}}{R^{2}}
$$

The radial coordinate of the shell is in the range $r_{+}<R<\infty$.

Equations (64) and (65), together with (66), can now be used to study the properties of the thin matter shells separating a Minkowski spacetime from an exterior extremal Reissner-Nordström spacetime, located outside the extremal gravitational radius $r_{+}$. In Eqs. (64) and (65) it is clear that it is necessary to pick the sign of $\xi$. Let us start with $\xi=+1$. It is useful to give the expressions for the shell's energy density and pressure, $\sigma$ and $p$. in terms of $M=Q$, where we opt for $M$. Using Eq. (22), i.e., $r_{+}=M$, in Eqs. (64) and (65) with $\xi=+1$ we have $8 \pi \sigma=\frac{M}{4 \pi R^{2}}$, $8 \pi p=0$, and also from Eq. (66) we have $8 \pi \sigma_{e}=\frac{2 M}{R^{2}}$. Let us now take $\xi=-1$. It is useful to give the expressions for the shell's energy density and pressure, $\sigma$ and $p$, in terms of $M=Q$, where as usual we opt for $M$. Using Eqs. (64) and (65) with $\xi=-1$ we have $8 \pi \sigma=\frac{2}{R}\left(2-\frac{M}{R}\right), 8 \pi p=-\frac{2}{R}$, and also from Eq. (66) we have again $8 \pi \sigma_{e}=\frac{2 M}{R^{2}}$. These are the expressions used in the two previous subsections. 


\section{EXTREMAL ELECTRIC THIN SHELLS INSIDE THE GRAVITATIONAL RADIUS: EXTREMAL TENSION SHELL REGULAR AND NONREGULAR BLACK HOLES AND MAJUMDAR-PAPAPETROU COMPACT NAKED SINGULARITIES}

\section{A. Extremal electric thin shells inside the event horizon: Extremal tension shell regular and nonregular black holes}

Here we study the case of a fundamental electric thin shell in the extremal state, i.e., $r_{+}=r_{-}$or $M=Q$, and indeed, $r_{+}=r_{-}=M=Q$, for which the shell's location obeys $R<r_{+}$, and so also $R<r_{-}$, and for which the orientation is such that the normal to the shell points towards $r_{+}$, i.e., we choose the quantity $\xi$ which gives the direction of the normal as $\xi=+1$, see the end of this section for details.. In this case horizons do exist and so, following the nomenclature, $r_{+}$is both the gravitational and the event horizon radius, and since $r_{+}=r_{-}$it is also the Cauchy horizon radius and the Cauchy radius. We opt to use $r_{+}$and $M$.

As functions of $M$ and $R$, the shell's energy density $\sigma$ and pressure $p$, are, see the end of this section,

$$
\begin{aligned}
& 8 \pi \sigma=\frac{2}{R}\left(2-\frac{M}{R}\right), \\
& 8 \pi p=-\frac{2}{R} .
\end{aligned}
$$

Also, the electric charge density $\sigma_{e}$ is given in terms of $M$ and $R$, by

$$
8 \pi \sigma_{e}=\frac{2 M}{R^{2}}
$$

The behavior of $\sigma$ and $p$, in Eqs. (67) and (68), as functions of the radial coordinate $R$ of the $\frac{Q}{M}=1$ extremal shell is shown in Figure 17. These shells are characterized by a positive energy density for $R$ near $r_{+}$that changes sign

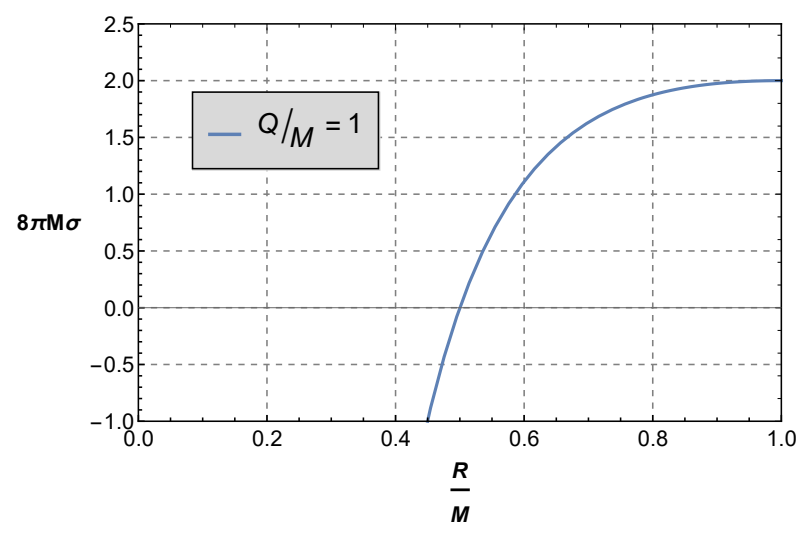

(a)

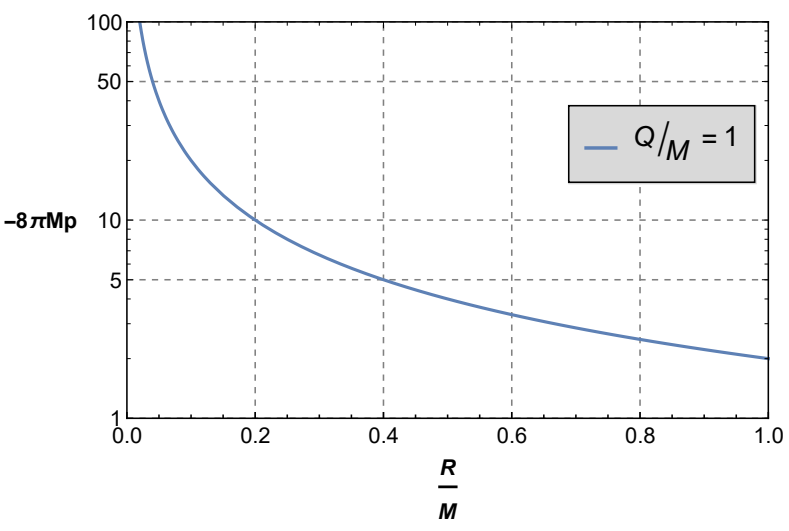

(b)

Figure 17: Physical properties of an extremal tension shell regular and nonregular black hole, i.e., an electric perfect fluid thin shell in an extremal Reissner-Nordström state, in the location $R<r_{+}=r_{-}$, i.e., located inside the

even hoizon, and with orientation such that the normal points towards $r_{+}$. The interior is Minkowski and the exterior is extremal Reissner-Nordström spacetime. Extremal means $\frac{Q}{M}=1$. Panel (a) Energy density $\sigma$ of the shell as a function of the radius $R$ of the shell. The energy density is adimensionalized through the mass $M, 8 \pi M \sigma$, and

the radius is adimensionalized through the gravitational radius $r_{+}, \frac{R}{r_{+}}$. Panel (b) Tension $-p$ on the shell as a function of the radius $R$ of the shell. The tension is adimensionalized through the mass $M,-8 \pi M p$, and the radius is adimensionalized through the event horizon radius $r_{+}, \frac{R}{r_{+}}$.

from positive to negative values when the radius of the shell $R$ obeys $R=\frac{M}{2}$ up to minus infinity when $R=0$. The exterior spacetime is extremal although $p$ is not zero and so the shell is not made of Majumdar-Papapetrou matter, this case providing thus another instance, of the many instances found in the literature, for which $M=Q$ globally but with an interior that is not made of Majumdar-Papapetrou matter. Equation (67) shows that $\sigma$ is the sum of a geometrical term given by $\frac{4}{R}$ and a gravitational term which is negative given by $-\frac{2 M}{R^{2}}$, wit the two terms being independent. The first term of $\sigma, \frac{4}{R}$, is a geometrical term that also gives rise to a geometrical tension given by $-\frac{2}{R}$ 
and ensures that there is a shell for sure with radius $R$ inside the Cauchy horizon $r_{+}=r_{-}$. This geometric term exists independently on whether there is spacetime mass $M$ or not, indeed, the spacetime mass energy coming from this geometrical term is zero since $\frac{4}{R}+2 p=0$. The second term $-\frac{2 M}{R^{2}}$ is negative and can be explained by the fact that inside a Cauchy horizon $r_{+}=r_{-}$gravity is repulsive, here manifested by $\sigma_{e}=\frac{2 M}{R^{2}}$, and since the shell is indeed inside $r_{+}=r_{-}$the shell tends naturally to $r_{+}$, so to counterbalance this effect and produce a static shell, the shell has to have an anti repulsive negative energy density, an anti Majumdar-Papapetrou energy density, of value $-\frac{2 M}{R^{2}}$. Note also that $\sigma+2 p+\sigma_{e}=0$. When $Q=0$, and so $M=0$, and since $R<M$, in the limiting case one has $R=0$, and we are left with a singular massless null shell at $R=0$ with $\sigma+2 p=0$ surrounded by a massless spacetime, i.e., a Minkowski spacetime. This Minkowski spacetime with a well defined singularity at its center is a new and interesting solution of Einstein equation. In relation to the energy conditions of the shell one can work out and find that the null, the weak, the dominant, and the strong energy conditions are never verified, see a detailed presentation ahead.

The Carter-Penrose diagram can be drawn directly from the building blocks of an interior Minkowski spacetime and the full extremal Reissner-Nordström spacetime. In Figure 18 two possible Carter-Penrose diagrams of a shell spacetime in an extremal Reissner-Nordström state, in the location $R<r_{+}=r_{-}$, with orientation such that the normal points towards $r_{+}$, are shown. It is clearly a black hole, more specifically, a tension shell black hole. In the diagram (a) the tension shell is inside the event horizon in region II. Then, in the next portion of the diagram there is another shell and so onwards. So, this realization it is a regular tension black hole. In the diagram (b) the tension shell is also inside the event horizon in region II. Then, the tension shell is replaced by the timelike singularity at $r=0$. So, in this realization it is a nonregular tension black hole. Since what one puts in the regions II, either a shell or a singularity, is not decided by the solution, an infinite number of different Carter-Penrose diagrams can be drawn, as there are an infinite number of combinations to locate a shell or a singularity when one goes upward or downward through the diagram. So, similarly to the previous subsection, in the case of shells whose unit normal points towards the event horizon, the maximal analytical extension of the spacetime may always contain a thin shell inside the event horizon or only at some regions.

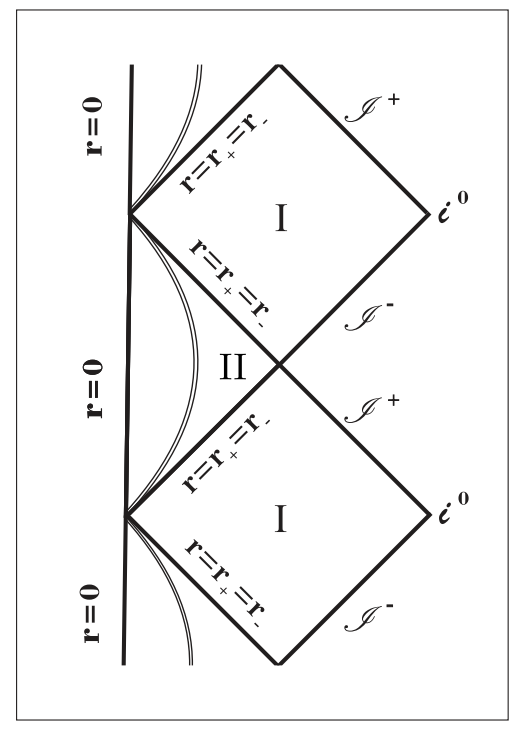

(a)

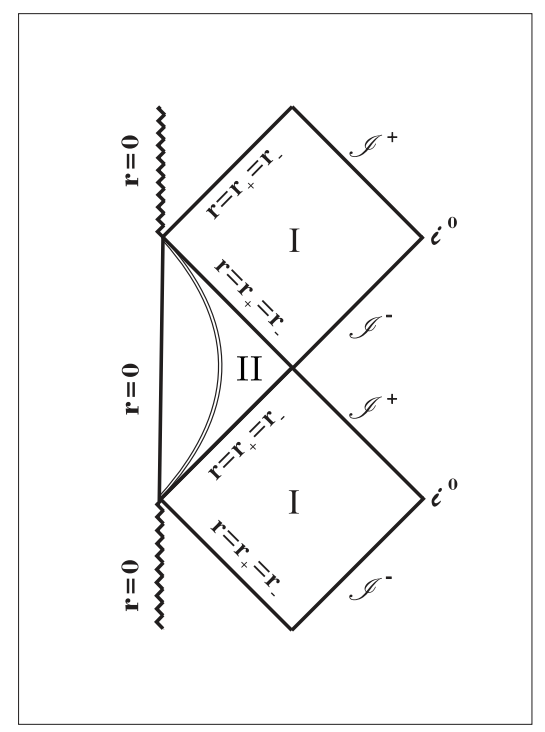

(b)

Figure 18: Carter-Penrose diagrams of the extremal tension shell black holes, i.e., a thin shell spacetime in an extremal Reissner-Nordström state, in the location $R<r_{+}=r_{-}$, i.e., located inside the event horizon radius, with orientation such that the normal to the shell points towards $r_{+}$. The interior is Minkowski, the exterior is extremal

Reissner-Nordström spacetime. Panel (a) The Carter-Penrose diagram contains a shell in the region II. If this pattern is repeated ad infinitum then it is an extremal tension shell regular black hole. Panel (b) The Carter-Penrose diagram contains a shell in region II and a singularity in regions II above and below. It is a tension shell black hole, now not regular. An infinite number of different Carter-Penrose diagrams can be drawn, since there are an infinite number of combinations to place the shell and the singularity.

The physical interpretation of this case is of some interest. This extremal thin shell solution provides an extremal regular black hole solution. The energy density and pressure never obey the energy conditions for all shell radii, i.e., shell radii between zero and the horizon. The causal and global structure as displayed by the Carter-Penrose diagram 
shows clearly that there is no singularity if one adopts the simplest form of the diagram. As regular extremal black holes these solutions join the other known regular black hole solutions which are of interest in quantum gravitational settings that presumably get rid of the singularities. So, this case falls into the category of having the energy conditions never verified, and in this sense is odd, although of interest as regular black hole matter solutions always are.

\section{B. Extremal electric thin shells inside the gravitational radius: Majumdar-Papapetrou compact shell naked singularities}

Here we study the case of a fundamental electric thin shell in the extremal state, i.e., $r_{+}=r_{-}$or $M=Q$, and indeed, $r_{+}=r_{-}=M=Q$, for which the shell's location obeys $R<r_{+}=r_{-}$, and for which the orientation is such that the normal to the shell points towards $r=0$, i.e., we choose the quantity $\xi$ which gives the direction of the normal as $\xi=-1$, see the end of this section for details.. In this case horizons do not exist and so, following the nomenclature, $r_{+}$is both the gravitational radius, and since $r_{+}=r_{-}$it is also the Cauchy radius. We opt to use $r_{+}$ and $M$.

As functions of $M$ and $R$, the shell's energy density $\sigma$ and pressure $p$, are, see the end of this section,

$$
\begin{aligned}
& 8 \pi \sigma=\frac{2 M}{R^{2}}, \\
& 8 \pi p=0
\end{aligned}
$$

Also, the electric charge density $\sigma_{e}$ is given in terms of $M$ and $R$ by Eq. (69). The behavior of $\sigma$ and $p$, in Eqs. (70) and (71), as functions of the radial coordinate $R$ of the $\frac{Q}{M}=1$ extremal shell is shown in Figure 19 . These shells are

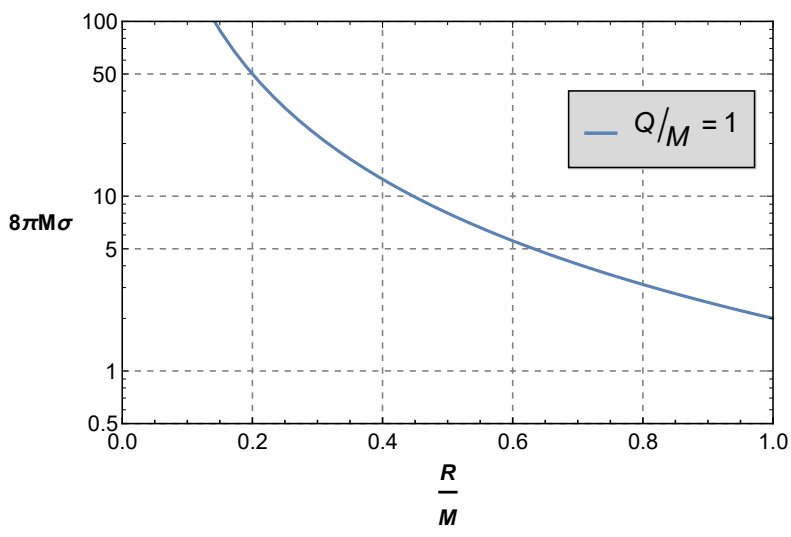

(a)

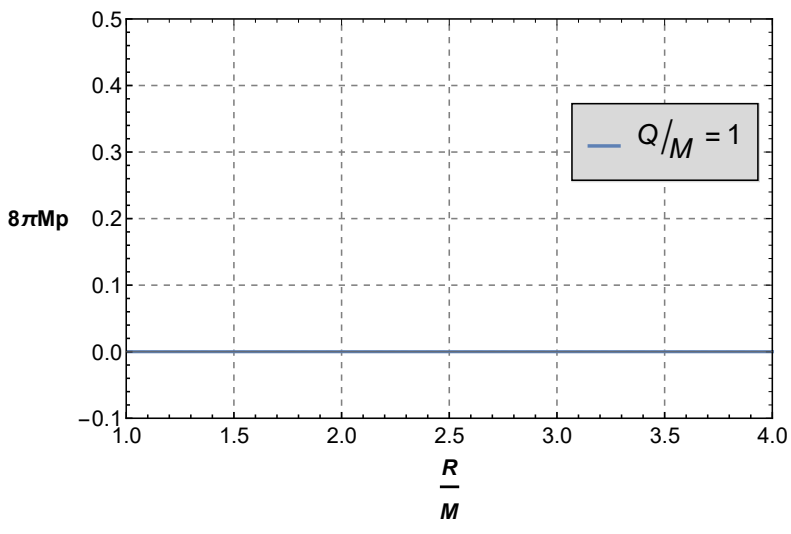

(b)

Figure 19: Physical properties of a Majumdar-Papapetrou compact shell naked singularity, i.e., an electric perfect fluid thin shell in an extremal Reissner-Nordström state, in the location $R<r_{+}=r_{-}$, and with orientation such that the normal points towards $r=0$. The interior is Minkowski and the exterior is extremal Reissner-Nordström spacetime. Extremal means $\frac{Q}{M}=1$. Panel (a) Energy density $\sigma$ of the shell as a function of the radius $R$ of the shell. The energy density is adimensionalized through the mass $M, 8 \pi M \sigma$, and the radius is adimensionalized through the gravitational radius $r_{+}, \frac{R}{r_{+}}$. Panel (b) Pressure on the shell as a function of the radius $R$ of the shell. The pressure is zero, the shell is supported by electric repulsion, it is Majumdar-Papapetrou matter. The radius is adimensionalized through the gravitational radius $r_{+}, \frac{R}{r_{+}}$.

characterized by a positive energy density for all shell's radii. The pressure is zero, and so the matter is MajumdarPapapetrou matter. When $Q=0$, and so $M=0$, there is no shell spacetime. In relation to the energy conditions of the shell one can work out and find that the null, the weak, the dominant, and the strong energy conditions are verified for $0<R<r_{+}$, see a detailed presentation ahead.

The Carter-Penrose diagram can be drawn directly from the building blocks of an interior Minkowski spacetime and the full extremal Reissner-Nordström spacetime. In Figure 20 the Carter-Penrose diagram of a shell spacetime in an extremal Reissner-Nordström state, in the location $R<r_{+}=r_{-}$, with orientation such that the normal points towards $r=0$, is shown. It is a Majumdar-Papapetrou, i.e., extremal, compact shell naked singularity spacetime. It is clearly a compact space, the coordinate $r$ goes from 0 to $R$ and then decreases back to 0 at the timelike singularity, 
such that there is no clear distinction of what is outside from what is inside. We use the hash symbol \# to represent the connected sum of the spacetime manifolds, in order to conserve the conformal structure in the Carter-Penrose diagram of the total spacetime.

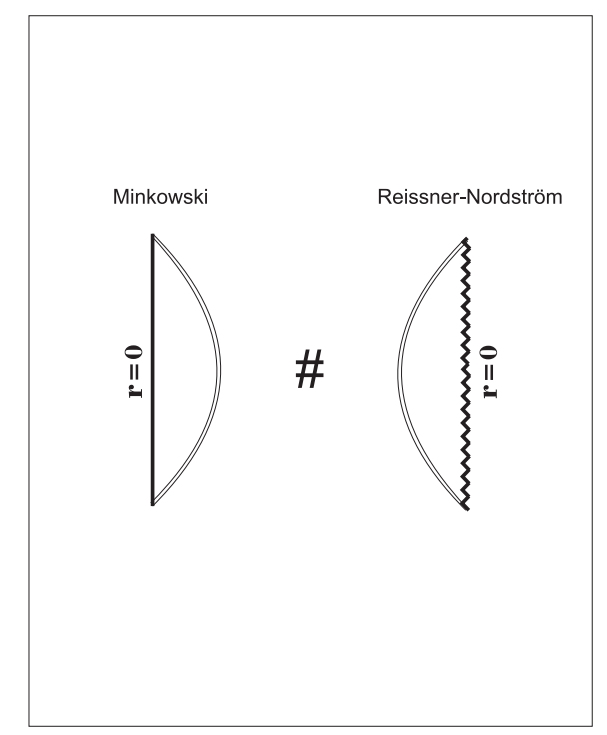

Figure 20: Carter-Penrose diagram of the Majumdar-Papapetrou compact shell naked singularity spacetime, i.e., a shell in an extremal Reissner-Nordström state, in the location $R<r_{+}=r_{-}$, i.e., located inside the event horizon radius, with orientation such that the normal to the shell points towards $r=0$. The interior is Minkowski, the exterior is extremal Reissner-Nordström. There is no clear distinction of what is outside from what is inside. The hash symbol \# represents the connected sum of the two spacetimes.

The physical interpretation of this case is noteworthy, and it is similar to the corresponding nonextremal shell. This extremal thin shell solution provides a closed spatial static universe with a singularity at one pole. There are no horizons. The energy density and pressure obey the energy conditions for all shell radii, indeed the shell is composed of Majumdar-Papapetrou matter. The causal and global structure as displayed by the Carter-Penrose diagram show the characteristics of this universe that has two sheets joined at the shell with one sheet having a singularity at its pole and with no horizons. The singularity is avoidable to timelike curves. So, this case falls into the category of having the energy conditions verified and the resulting spacetime being peculiar.

\section{Formalism for extremal electric thin shells inside the gravitational radius}

\section{Preliminaries}

We now make a careful study to derive the properties of the fundamental electric thin shell used in the two previous subsections, i.e., the thin shell in an extremal state, i.e., $r_{+}=r_{-}$or $M=Q$, for which the shell's radius $R$ location obeys $R<r_{+}=r_{-}$, and for which the orientation is such that the normal to the shell points towards $r_{+}$or towards $r=0$. It should be read as an appendix to the previous two subsections. We use the formalism developed in Sec. II.

\section{Induced metric, and extrinsic curvature of $\mathcal{S}$ as seen from $\mathcal{M}_{\mathrm{i}}$}

Let us start by analyzing the interior Minkowski spacetime, $\mathcal{M}_{\mathrm{i}}$. Since it is the same as the analysis done previously we only quote the important equations. They are the interior metric Eq. (28), the interior four-velocity of the shell Eq. (29), the metric for the shell at radius $R$ Eq. (30), the normal to the shell Eq. (31), and the extrinsic curvature from the inside Eq. (32). 


\section{Induced metric, and extrinsic curvature of $\mathcal{S}$ as seen from $\mathcal{M}_{\mathrm{e}}$}

To proceed we have now to find the expressions for the induced metric on $\mathcal{S}$ and the extrinsic curvature components as seen from the exterior spacetime, $\mathcal{M}_{\mathrm{e}}$, in the extremal state, i.e., $r_{+}=r_{-}$or $M=Q$, see Figure 3 , for which the shell's obeys $R<r_{+}=r_{-}$, and for which the orientation is such that the normal to the shell points towards increasing $r$, i.e., towards $r_{+}$, or towards decreasing $r$ i.e., towards $r=0$, as seen from the exterior, as used in the two previous subsections.

Most of the analysis and results of Section $V$ are still verified, namely, the extremal Reissner-Nordström line element $d s_{\mathrm{e}}^{2}$ given in Eq. (59), the four-velocity $u_{\mathrm{e}}^{\alpha}$ given in Eq. (60), the line element on $\mathcal{S},\left.d s^{2}\right|_{\mathcal{S}}$ given in Eq. (61), and the normal to the surface $\mathcal{S}$ given in Eq. (62). Then, taking into account that here we are considering that, $R$, the radial coordinate of $\mathcal{S}$ as seen from $\mathcal{M}_{\mathrm{e}}$, verifies $R<r_{+}=r_{-}$, we find the following expressions for the nonzero components of the extrinsic curvature of the matching hypersurface

$$
K_{\mathrm{e}}^{\tau} \tau=-\xi \frac{r_{+}}{R^{2}}, \quad K_{\mathrm{e}}{ }^{\theta} \theta=K_{\mathrm{e}}^{\varphi}{ }_{\varphi}=\xi \frac{k}{R},
$$

where, as before, the parameter $\xi$ is defined as $\xi=+1$ if the orientation is such that the outside unit normal to the shell points in the direction of increasing radial coordinate $r$, measured by an observer in the exterior $\mathcal{M}_{\mathrm{e}}$ spacetime, and $\xi=-1$ if the the orientation is such that the outside unit normal to the shell points in the direction of decreasing radial coordinate $r$, and the redshift function $k$ at the shell is given by $k=\left|1-\frac{r_{+}}{R}\right|$, i.e., since $R<r_{+}$one has $k=\frac{r_{+}}{R}-1$.

\section{Shell's energy density and pressure}

Having determined the components of the extrinsic curvature of the matching surface $\mathcal{S}$ as seen from the interior and exterior spacetimes we are now in position to use the second junction condition given in Eq. (9) to find the expressions for the energy density and pressure support of the thin shell. The shell's stress-energy tensor is given in Eq. (10), and Eqs. (32) and (72) then yield

$$
\begin{aligned}
& 8 \pi \sigma=\frac{2}{R}\left[1+\xi\left(1-\frac{r_{+}}{R}\right)\right], \\
& 8 \pi p=-\frac{1}{R}(1+\xi),
\end{aligned}
$$

where we used $k=\frac{r_{+}}{R}-1=\frac{M}{R}-1$. Note that $p$ in Eq. (65) is independent of $M$, it only depends on $R$ and thus on the geometry of the shell as embedded in the ambient spacetime. Moreover, defining the surface electric current density $s_{a}$ on the thin shell as $s_{a}=\sigma_{e} u_{a}$, where $\sigma_{e}$ represents the electric charge density, and since the Minkowski spacetime has zero electric charge, from Eqs. (12)-(13) and (21) it follows that

$$
8 \pi \sigma_{e}=2 \frac{r_{+}}{R^{2}} .
$$

The radial coordinate of the shell is in the range $0<R<r_{+}$.

Equations (73) and (74), together with (75), can now be used to study the properties of the thin matter shells separating a Minkowski spacetime from an exterior extremal Reissner-Nordström spacetime, located inside the event horizon $r_{+}$. In Eqs. (73) and (74) it is clear that it is necessary to pick the sign $\xi$. Let us start with $\xi=+1$. It is useful to give the expressions for the shell's energy density and pressure, $\sigma$ and $p$ in terms of $M=Q$, we opt for $M$. Using Eq. (22), i.e., $r_{+}=M$, in Eqs. (73) and (74) with $\xi=+1$ we have $8 \pi \sigma=\frac{2}{R}\left(2-\frac{M}{R}\right), 8 \pi p=-\frac{2}{R}$, and also from Eq. (66) we have $8 \pi \sigma_{e}=\frac{2 M}{R^{2}}$. Let us now take $\xi=-1$. It is useful to give the expressions for the shell's energy density and pressure, $\sigma$ and $p$ in terms of $M=Q$, we opt for $M$. Using Eqs. (73) and (74) with $\xi=-1$ we have $8 \pi \sigma=\frac{2 M}{R^{2}}, 8 \pi p=0$, and also from Eq. (66) we have again $8 \pi \sigma_{e}=\frac{2 M}{R^{2}}$. These are the expressions used in the two previous subsections. 


\section{EXTREMAL ELECTRIC THIN SHELLS AT THE GRAVITATIONAL RADIUS: MAJUMDAR-PAPAPETROU SHELL QUASIBLACK HOLES, EXTREMAL NULL SHELL QUASINONBLACK HOLES, EXTREMAL NULL SHELL SINGULARITIES, AND MAJUMDAR-PAPAPETROU NULL SHELL SINGULARITIES}

\section{A. Extremal electric thin shells at the event horizon: Majumdar-Papapetrou shell quasiblack holes and} extremal null shell quasinonblack holes

\section{Majumdar-Papapetrou shell quasiblack holes}

Here we study the case of a fundamental electric thin shell in the extremal state, i.e., $r_{+}=r_{-}$or $M=Q$, and indeed, $r_{+}=r_{-}=M=Q$, for which the shell's location obeys $R=r_{+}$, and for which the orientation is such that the normal to the shell points towards spatial infinity. Moreover, there is an additional characterization for shells at the horizon. This case comes from the limit of $R \rightarrow r_{+}$from above and so is the limiting case of the case studied in Sec.V A. In this case a horizon is barely formed, namely, we have a quasihorizon, and so, following the nomenclature, $r_{+}$is both the gravitational radius and the quasihorizon radius. This is an extremal quasiblack hole [37]. Also $r_{+}$and $r_{-}$have the same value. In general we also opt to use $M$ rather than $Q$. The normal to the shell pointing towards spatial infinity means in the notation for the extremal states that the new parameter $\xi$ has value $\xi=+1$, see the end of this section for details.

As functions of $M$ and $R$, the shell's energy density $\sigma$ and pressure $p$, are, see the end of this section,

$$
\begin{aligned}
& 8 \pi \sigma=\frac{2}{M}, \\
& 8 \pi p=0 .
\end{aligned}
$$

Also, the electric charge density $\sigma_{e}$ is given in terms of $M$ by

$$
8 \pi \sigma_{e}=\frac{2}{M} .
$$

Since it is one point in a plot of $\sigma$ or $p$ as functions of $\frac{R}{M}$, there is no need to draw a figure. The shell is characterized by a positive energy density. The pressure is zero, and so the matter is Majumdar-Papapetrou matter, i.e., $\sigma_{e}=\sigma$, and therefore is fully supported by electric repulsion. This is an interesting system to consider, this case when the shell's radius is taken to the event horizon radius. It is a quasiblack hole configuration. The Majumdar-Papapetrou shell quasiblack hole is regular in that all curvature scalars are finite everywhere. When $Q=0$, so $M=0$ and $r_{+}=0$, the shel is at $R=0$, and the spacetime is singular being Minkowski in the exterior. In relation to the energy conditions of the shell one can work out and find that the null, the weak, the dominant, and the strong energy conditions are always verified, see a detailed presentation ahead.

The Carter-Penrose diagram can be drawn with some care from the building blocks of an interior Minkowski spacetime and the exterior asymptotic region of an extremal Reissner-Nordström spacetime, see [31] and for more details see [37]. In Figure 21 the Carter-Penrose diagram of a Majumdar-Papapetrou shell quasiblack hole, i.e., for $R=r_{+}$and a junction surface with orientation such that the outside normal points towards spacial infinity is shown. We use the hash symbol \# to represent the connected sum of the spacetime manifolds, in order to conserve the conformal structure in the Carter-Penrose diagram of the total spacetime. We see that when the shell is at $R=r_{+}$, i.e., the shell is at a null surface, the two regions contain incomplete geodesics with ending points at the matching surface, so that, observers at each spacetime are disconnected and the manifold is composed by two separate regions. 


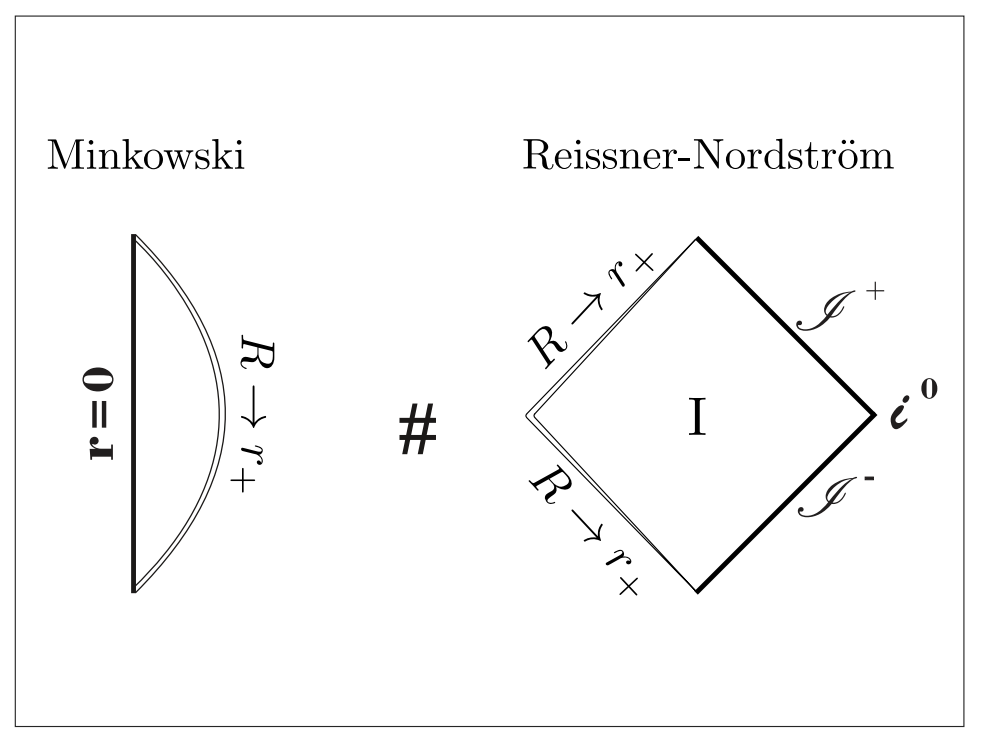

Figure 21: Carter-Penrose diagram of a Majumdar-Papapetrou shell quasiblack hole, i.e., a thin shell spacetime in an extremal Reissner-Nordström state, with the shell located at $R=r_{+}$, i.e., located at the gravitational radius or quasihorizon, with orientation such that the normal points towards infinity, and such that $R \rightarrow r_{+}$from $R>r_{+}$. The interior is Minkowski, the exterior is extremal Reissner-Nordström. This quasiblack hole shell is supported by electrical repulsion alone.

Some remarks on quasiblack holes should be made. In this section we treated an extremal quasiblack hole, namely, a Majumdar-Papapetrou shell quasiblack hole. Since it is Majumdar-Papapetrou the pressure on the shell is zero, $p=0$, and so the extremal quasiblack hole is regular in this sense. On the other hand, in Section III A on nonextremal shells that are located outside $r_{+}, R>r_{+}$, with orientation such that the normal points towards spatial infinity, one has that Eqs. (23), (24), and (25), in the limit that the shell is located at the gravitational radius, $R=r_{+}$, yield that the surface density $\sigma$ is finite, the pressure support $p$ of the thin matter shell diverges to infinity, and the electric charge density $\sigma_{e}$ is finite. This case defines a nonextremal quasiblack hole. Since the pressure diverges the spacetime of nonextremal shells at $R=r_{+}$presents some type of singularity. This singularity is mild however, with entropy and the mass formulas being derived in this limiting case, see [37]. The Carter-Penrose diagram of a nonextremal quasiblack hole is similar to the Carter-Penrose diagram for a Majumdar-Papapetrou one, i.e., the one showed in Figure 21. Since nonextremal quasiblack holes are somewhat singular and extremal ones are not, we have treated these within the extremal state and mentioned the nonextremal here.

The physical interpretation of this case is known and it is remarkable. The extremal thin shell solution with its radius at the horizon radius, is inherited from the extremal thin shell star, and provides a typical extremal quasiblack hole. A quasiblack hole is an object on the verge of becoming a black hole, but cannot turn into such one. The energy density and pressure shows that the matter is Majumdar-Papapetrou and obey the energy conditions. The causal and global structure as displayed by the Carter-Penrose diagram show the quasiblack hole characteristics. These quasiblack holes have no curvature singularities, although at the quasihorizon there is some form of singular degeneracy that disconnects the interior from the exterior. It can form in a limiting process of quasistatic collapse. Quasiblack holes are of great interest because they reveal new black hole properties or black hole properties in a new perspective. So, this case falls into the category of having some of the energy conditions verified and the geometrical setup is interesting and peculiar.

\section{Extremal null shell quasinonblack holes}

Here we study the case of a fundamental electric thin shell in the extremal state, i.e., $r_{+}=r_{-}$or $M=Q$, and indeed, $r_{+}=r_{-}=M=Q$, for which the shell's location obeys $R=r_{+}$, and for which the orientation is such that the normal to the shell points towards spatial infinity. Moreover, there is an additional characterization for shells at the horizon. This case here comes from the limit of $R \rightarrow r_{+}$from below and so is the limiting case of the case studied in Sec.VI A. In this case $r_{+}$is timelike on one side and lightlike on the other side. Thus, a horizon, or rather a quasinonhorizon, does exist and so, following the nomenclature, $r_{+}$is both the gravitational radius and the 
quasinonhorizon radius. Also $r_{+}$and $r_{-}$have the same value. In general, we also opt here to use $M$ rather than $Q$. The normal to the shell pointing towards spatial infinity means in the notation for the extremal states that the new parameter $\xi$ has value $\xi=+1$, see the end of this section for details.

As functions of $M$ and $R$, the shell's energy density $\sigma$ and pressure $p$, are, see the end of this section,

$$
\begin{aligned}
8 \pi \sigma & =\frac{2}{M}, \\
8 \pi p & =-\frac{2}{M} .
\end{aligned}
$$

Also, the electric charge density $\sigma_{e}$ is given in terms of $M$ by Eq. (78). Since it is one point in a plot of $\sigma$ or $p$ as functions of $\frac{R}{M}$, there is no need to draw a figure. The shell is characterized by a positive energy density. The pressure is negative, so it is a tension. The equation of state is $\sigma+2 p+\sigma_{e}=0$, inherited from the extremal $R<r_{+}$shell. When $Q=0$, and so $M=0$, there is a singular null shell at $R=0$, and a Minkowski spacetime in the exterior. In relation to the energy conditions of the shell one can work out and find that the null, the weak and the dominant energy conditions are always verified, and the strong energy condition is always violated, see a detailed presentation ahead.

The Carter-Penrose diagram can be drawn with some care from the building blocks of an interior Minkowski spacetime and the exterior asymptotic region of an extremal Reissner-Nordström spacetime. In Figure 22, the CarterPenrose diagram of an extremal null shell quasinonblack hole, i.e., for $R=r_{+}$and a junction surface with orientation such that the outside normal points towards spatial infinity, is shown. We use the hash symbol \# to represent the connected sum of the spacetime manifolds, in order to conserve the conformal structure in the Carter-Penrose diagram of the total spacetime. This setup is very different from the quasiblack hole limit of the last section leading to a new Carter-Penrose diagram. Nonetheless, we see that as in the previous case, when the shell is at $R=r_{+}$, i.e., the shell, for one of the regions, is at a null surface, the two regions contain incomplete geodesics with ending points at the matching surface, so that, observers at each spacetime are disconnected and the manifold is composed by two separate regions.

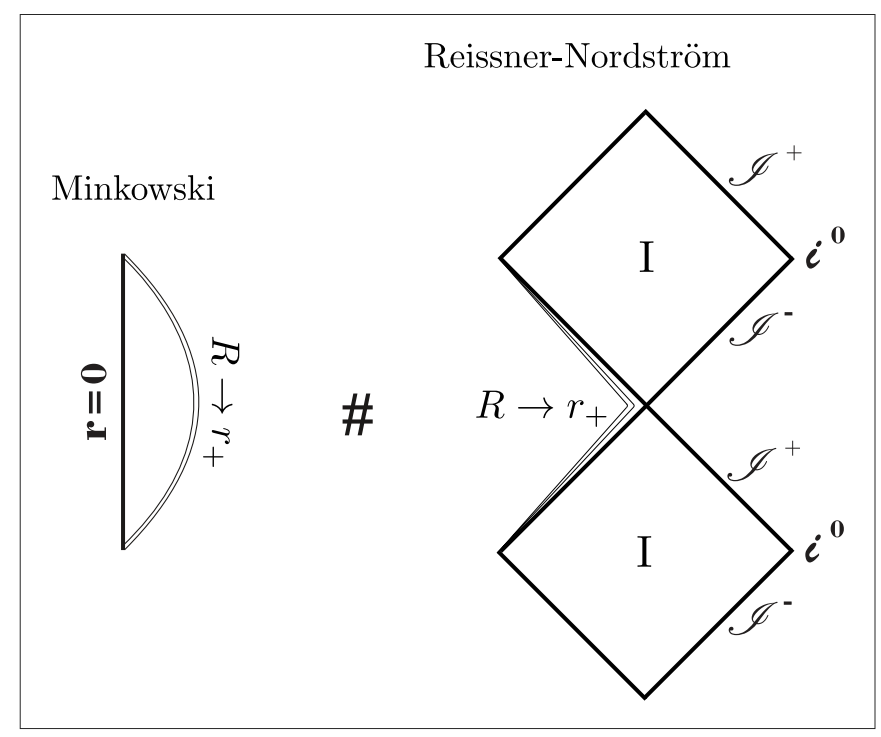

Figure 22: Carter-Penrose diagram of an extremal null shell quasinonblack hole, i.e., a thin shell spacetime in an extremal Reissner-Nordström state, with the shell located at $R=r_{+}$, i.e., located at the gravitational radius or horizon, with orientation such that the normal points towards $r=0$, and such that $R \rightarrow r_{+}$from $R<r_{+}$. The interior is Minkowski, the exterior is extremal Reissner-Nordström.

The physical interpretation of this case is also remarkable. The extremal thin shell solution with its radius at the horizon radius, is inherited from the extremal regular black hole, and provides an example of an extremal quasinonblack hole It is an object that is on the verge of becoming a star solution, but cannot turn into one. The energy density and pressure shows that the matter obeys some of the energy conditions. These quasinonblack holes have no curvature singularities, although at the quasinonhorizon there is some form of singular degeneracy that disconnects the interior from the exterior. The causal and global structure as displayed by the Carter-Penrose diagram show the characteristics pertaining to quasinonblack hole. These quasinonblack hole solutions are new, they have showed up here for the first 
time. So, this case falls into the category of having the energy conditions verified and the geometrical setup is new, very interesting, and peculiar.

\section{B. Extremal electric thin shells at the gravitational radius: Extremal null shell singularities, and Majumdar-Papapetrou null shell singularities}

\section{Extremal null shell singularities}

Here we study the case of a fundamental electric thin shell in the extremal state, i.e., $r_{+}=r_{-}$or $M=Q$, and indeed, $r_{+}=r_{-}=M=Q$, for which the shell's location obeys $R=r_{+}$, and for which the orientation is such that the normal to the shell points towards the singularity at $r=0$. Moreover, as we have seen above, there is an additional characterization for shells at the horizon, this case comes from the limit of $R \rightarrow r_{+}$from above and so is the limiting case of the case studied in Sec.VB. In this case the shell is at the horizon, thus in a sense a quasihorizon does exist, and so, following the nomenclature, $r_{+}$is both the gravitational radius and the quasihorizon radius. Also $r_{+}$and $r_{-}$ have the same value. In general we also opt to use $M$ rather than $Q$. This is an extremal null shell singularity. The normal to the shell pointing towards the singularity at $r=0$ means in the notation for the extremal states that the new parameter $\xi$ has value $\xi=-1$, see the end of this section for details.

As functions of $M$ and $R$, the shell's energy density $\sigma$ and pressure $p$, are, see the end of this section,

$$
\begin{aligned}
& 8 \pi \sigma=\frac{2}{M}, \\
& 8 \pi p=-\frac{2}{M},
\end{aligned}
$$

so the matter is not Majumdar-Papapetrou. Also, the electric charge density $\sigma_{e}$ is given in terms of $M$ by Eq. (78). The equation of state is $\sigma+2 p+\sigma_{e}=0$, inherited from the extremal $R<r_{+}$shell. In relation to the energy conditions of the shell one can work out and find that the null, the weak and the dominant energy conditions are always verified whereas, the strong energy condition is never verified, see a detailed presentation ahead.

The Carter-Penrose diagram can be drawn with some care from the building blocks of an interior Minkowski spacetime and the exterior asymptotic region of an extremal Reissner-Nordström spacetime. In Figure 23 the CarterPenrose diagram of an extremal null shell singularity, i.e., for $R=r_{+}$from above and a junction surface with orientation such that the outside normal points towards the singularity at $r=0$ is shown. We see that when the shell is at $R=r_{+}$, that is the shell is at a null surface, the two regions contain incomplete geodesics with ending points at the matching surface, so that, observers at each spacetime are disconnected and the manifold is composed by two separate regions.

The physical interpretation of this case follows from the corresponding extremal shell outside the gravitational radius. This extremal thin shell solution, with the shell itself at the horizon, or more properly, at the quasinonhorizon, turns the space around at the quasinonhorizon and then ends in a singularity. The energy density and pressure obey some of the energy conditions. The causal and global structures as displayed by the Carter-Penrose diagram are interesting and the two parts up to the shell and from the shell to the singularity are disjoint, with the quasinonhorizon presenting some form of degeneracy, although there are no curvature singularities there. So, this case falls into the category of having some of the energy conditions verified and the geometrical setup is rather strange. 


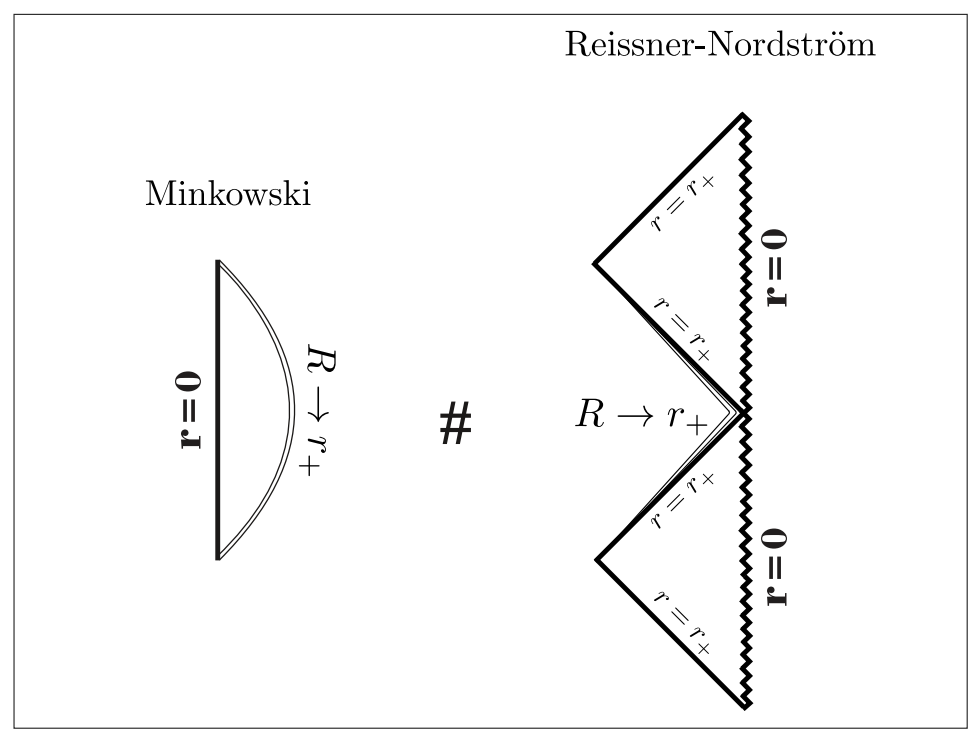

Figure 23: Carter-Penrose diagram of an extremal null shell singularity, i.e., a thin shell spacetime in an extremal Reissner-Nordström state, with the shell located at $R=r_{+}$, i.e., located at the gravitational radius from above, with orientation such that the normal points towards the singularity at $r=0$, and such that $R \rightarrow r_{+}$from $R>r_{+}$. The interior is Minkowski, the exterior is extremal Reissner-Nordström.

\section{Majumdar-Papapetrou null shell singularities}

Here we study the case of a fundamental electric thin shell in the extremal state, i.e., $r_{+}=r_{-}$or $M=Q$, and indeed, $r_{+}=r_{-}=M=Q$, for which the shell's location obeys $R=r_{+}$, and for which the orientation is such that the normal to the shell points towards the singularity at $r=0$. Moreover, there is an additional characterization for shells at the horizon, this case comes from the limit of $R \rightarrow r_{+}$from below and so is the limiting case of the case studied in Sec.VI B. In this case there is a null shell, which is not a horizon, and so, following the nomenclature, $r_{+}$is the gravitational radius. Also $r_{+}$and $r_{-}$have the same value. In general we also opt to use $M$ rather than $Q$. The normal to the shell pointing towards $r=0$ means in the notation for the extremal states that the new parameter $\xi$ has value $\xi=-1$, see the end of this section for details.

As functions of $M$ and $R$, the shell's energy density $\sigma$ and pressure $p$, are, see the end of this section,

$$
\begin{aligned}
& 8 \pi \sigma=\frac{2}{M}, \\
& 8 \pi p=0 .
\end{aligned}
$$

Also, the electric charge density $\sigma_{e}$ is given in terms of $M$ by Eq. (78). Since it is one point in a plot of $\sigma$ or $p$ as functions of $\frac{R}{M}$, there is no need to draw a figure. The shell is characterized by a positive energy density. The pressure is zero, and so the matter is Majumdar-Papapetrou matter, i.e., $\sigma_{e}=\sigma$, and therefore is fully supported by electric repulsion. When $Q=0$, and so $M=0$, there is a singularity at $R=0$ and Minkowski in the exterior. In relation to the energy conditions of the shell one can work out and find that the null, the weak, the dominant, and the strong energy conditions are always verified, see a detailed presentation ahead.

The Carter-Penrose diagram can be drawn with some care from the building blocks of an interior Minkowski spacetime and the exterior asymptotic region of an extremal Reissner-Nordström spacetime. In Figure 24 the CarterPenrose diagram of an extremal Majumdar-Papapetrou shell singularity, i.e., for $R=r_{+}$from below and a junction surface with orientation such that the outside normal points towards $r=0$ is shown. The two regions contain complete geodesics so that the manifold is composed by two connected regions, where in the interior there is Minkowski spacetime, and on the exterior extremal Reissner-Nordström spacetime. 


\section{Minkowski}

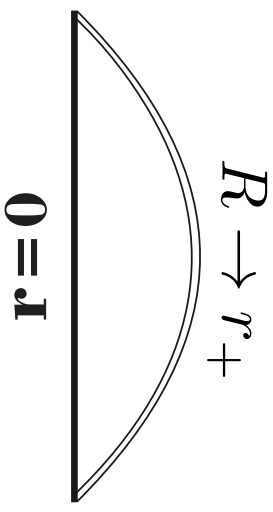

\section{Reissner-Nordström}

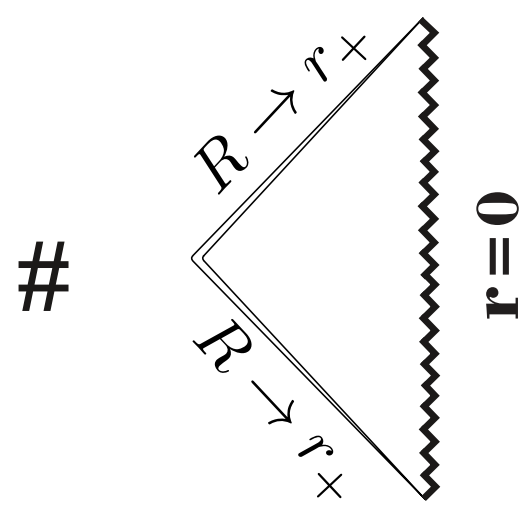

Figure 24: Carter-Penrose diagram of an extremal Majumdar-Papapetrou null shell singularity, i.e., a thin shell spacetime in an extremal Reissner-Nordström state, with the shell located at $R=r_{+}$, i.e., located at the gravitational radius, with orientation such that the normal points towards $r=0$, and such that $R \rightarrow r_{+}$from $R<r_{+}$. The interior is Minkowski, the exterior is extremal Reissner-Nordström.

The physical interpretation of this case follows from the corresponding extremal shell inside the gravitational radius. This extremal thin shell solution provides a closed spatial static universe with a singularity at one pole. There are quasihorizons. The energy density and pressure obey the energy conditions for all shell radii, indeed the shell is composed of Majumdar-Papapetrou matter. The causal and global structure as displayed by the Carter-Penrose diagram show the characteristics of this universe that has two sheets joined at the shell. For one sheet, i.e., for one side of the universe, the shell is timelike, for the other sheet, the shell is null, and possesses a timelike singularity. So, this case falls into the category of having the energy conditions verified and the resulting spacetime being strange.

\section{Formalism for extremal electric thin shells at the gravitational radius}

\section{Preliminaries}

We now make a careful study to derive the properties of the fundamental electric thin shell used in the two previous subsections, i.e., the thin shell in an extremal state, i.e., $r_{+}=r_{-}$or $M=Q$, for which the shell's radius $R$ location obeys $R=r_{+}=r_{-}$, and for which the orientation is such that the normal to the shell points towards infinity or towards $r=0$. It should be read as an appendix to the previous two subsections. We use the formalism developed in Sec. II.

\section{Induced metric, and extrinsic curvature of $\mathcal{S}$ as seen from $\mathcal{M}_{\mathrm{i}}$}

Let us start by analyzing the interior Minkowski spacetime, $\mathcal{M}_{\mathrm{i}}$. Since it is the same as the analysis done previously we only quote the important equations. They are the interior metric Eq. (28), the interior four-velocity of the shell Eq. (29), the metric for the shell at radius $R$ Eq. (30), the normal to the shell Eq. (31), and the extrinsic curvature from the inside Eq. (32).

\section{Induced metric, and extrinsic curvature of $\mathcal{S}$ as seen from $\mathcal{M}_{\mathrm{e}}$}

To proceed we have now to find the expressions for the induced metric on $\mathcal{S}$ and the extrinsic curvature components as seen from the exterior spacetime, $\mathcal{M}_{\mathrm{e}}$, in the extremal state, i.e., $r_{+}=r_{-}$or $M=Q$, see Figure 3 , for which the radius of the shell $R$ tends towards $r_{+}=r_{-}$, and for which the orientation is such that the normal to the shell 
points towards increasing $r$, i.e., towards spatial infinity, or towards decreasing $r$, i.e., towards $r=0$, as seen from the exterior, as we considered in the two previous subsections. Moreover, besides the direction of the normal as seen from the exterior spacetime, we also have to differentiate between the cases when the shell is located outside or inside the event horizon, i.e., $R>r_{+}$, or $R<r_{+}$, see Sec. V and VI, respectively.

The direction of the normal is taken into account by the parameter $\xi$ as previously used. In order to account for the two possibilities $R>r_{+}$and $R<r_{+}$when $R$ tends to $r_{+}$, we introduce a new sign parameter $\chi$ defined by $\chi=\operatorname{sign}\left(R-r_{+}\right)$. Then, we can take directly from Eqs. (63) and (72) the expressions for the extrinsic curvature

$$
K_{\mathrm{e}}^{\tau} \tau=\chi \xi \frac{1}{r_{+}}, \quad K_{\mathrm{e}}{ }^{\theta}{ }_{\theta}=K_{\mathrm{e}}{ }^{\varphi} \varphi=\xi \frac{k}{R},
$$

where again $\xi$ is defined as $\xi=+1$ if the outside unit normal to the shell points in the direction of increasing radial coordinate $r$, measured by an observer in the exterior $\mathcal{M}_{\mathrm{e}}$ spacetime, and $\xi=-1$ if the outside unit normal to the shell points in the direction of decreasing radial coordinate $r$, and $k=\left|1-\frac{r_{+}}{R}\right|$.

\section{Shell's energy density and pressure}

Having determined the components of the extrinsic curvature of the matching surface $\mathcal{S}$ as seen from the interior and exterior spacetimes we are now in position to use the second junction condition (9) to find the expressions for the energy density and pressure support of the thin shell. The relations $\sigma=-\frac{1}{4 \pi}\left[K_{\theta}^{\theta}\right]$ and $p=\frac{1}{8 \pi}\left[K_{\tau}^{\tau}\right]-\frac{\sigma}{2}$ then yield

$$
\begin{aligned}
& 8 \pi \sigma=\frac{2}{r_{+}}, \\
& 8 \pi p=\frac{\xi}{r_{+}}(\chi-\xi) .
\end{aligned}
$$

Moreover, defining the surface electric current density $s_{a}$ on the thin shell as $s_{a}=\sigma_{e} u_{a}$, where $\sigma_{e}$ represents the electric charge density, and since the Minkowski spacetime has zero electric charge, from Eqs. (12)-(13) and (21) it follows that

$$
8 \pi \sigma_{e}=\frac{2}{r_{+}}
$$

The radial coordinate of the shell is $R=r_{+}$.

In Eq. (87) it is clear that it is necessary to pick the signs of $\xi$ and $\chi$. It is useful to give the expressions for the shell's energy density and pressure, $\sigma$ and $p$ in terms of $M=Q$, where as usual we opt for $M$. Using Eq. (22), i.e., $r_{+}=M$, in Eqs. (86)-(88) with $\xi=+1$ and $\chi=+1$ we have $8 \pi \sigma=\frac{2}{M}, 8 \pi p=0$, and $8 \pi \sigma_{e}=\frac{2}{M}$. Choosing now $\xi=+1$ and $\chi=-1$, with $r_{+}=M$, in Eqs. (86)-(88) we have $8 \pi \sigma=\frac{2}{M}, 8 \pi p=-\frac{2}{M}$, and $8 \pi \sigma_{e}=\frac{2}{M}$. Choosing then $\xi=-1$ and $\chi=+1$, with $r_{+}=M$, in Eqs. (86)-(88) we have $8 \pi \sigma=\frac{2}{M}, 8 \pi p=-\frac{2}{M}$, and $8 \pi \sigma_{e}=\frac{2}{M}$. Choosing finally $\xi=-1$ and $\chi=-1$, with $r_{+}=M$, in Eqs. (86)-(88) we have $8 \pi \sigma=\frac{2}{M}, 8 \pi p=0$, and $8 \pi \sigma_{e}=\frac{2}{M}$. These are the expressions used in the two previous subsections to study the properties of the thin matter shells located at the event horizon $r_{+}$separating a Minkowski spacetime from an exterior extremal Reissner-Nordström spacetime. 


\section{OVERCHARGED ELECTRIC THIN SHELLS: OVERCHARGED STAR SHELLS AND COMPACT OVERCHARGED SHELL NAKED SINGULARITIES}

\section{A. Overcharged electric thin shells: Overcharged star like shells}

Here we study the case of a fundamental electric thin shell in the overcharged state, i.e., $r_{+}$and $r_{-}$are not real, or $M<Q$, for which the shell's location is anywhere, i.e., $0<R<\infty$, and for which the orientation is such that the normal to the shell points towards spatial infinity. In this case horizons do not exist and moreover $r_{+}$and $r_{-}$do not exist, and so there is neither gravitational radius nor Cauchy radius. The normal to the shell pointing towards spatial infinity means in the notation we have been using that $\xi=+1$, see the end of this section for details.

As functions of $M, Q$, and $R$, the shell's energy density $\sigma$ and pressure $p$, are, see the end of this section,

$$
\begin{gathered}
8 \pi \sigma=\frac{2}{R}(1-k), \\
8 \pi p=\frac{1}{2 R k}\left[(1-k)^{2}-\frac{Q^{2}}{R^{2}}\right],
\end{gathered}
$$

respectively, with $k=\sqrt{1-\frac{2 M}{R}+\frac{Q^{2}}{R^{2}}}$. Also, the electric charge density $\sigma_{e}$ is given in terms of $M, Q$, and $R$, by

$$
8 \pi \sigma_{e}=\frac{2 Q}{R^{2}}
$$

The behavior of $\sigma$ and $p$ as functions of the radial coordinate $R$ of the shell for various values of the $\frac{Q}{M}$ ratio in this case is shown in Figure 25. From Figure 25 we see that, depending on the radial coordinate of the shell, the energy density might take negative values. Indeed, from Eq. (89) we find that for $R<\frac{Q^{2}}{2 M}$ the energy density $\sigma$ is negative. Also, this case of thin shell is always supported by negative pressure, i.e., tension, see Eq. (90). It is a tension shell and can also be a negative energy density shell. The fact that it is supported by negative energy density sometimes and by tension translates the well known fact that the Reissner-Nordström singularity at $r=0$ is repulsive. Moreover, we see that both the energy density and the pressure of the shell diverge to negative infinity as the shell gets closer to $R=0$. On the other hand, in the limit of $R \rightarrow \infty$ both energy density and the pressure go to zero. When $Q=0$ there are no shells, since then $M=0$ as we are not considering negative $M$. In relation to the energy conditions of the shell we can say that the null, the weak, and the dominant, energy conditions are verified when $R \geq R_{\mathrm{I}^{\prime}}$, and the strong energy condition is verified when $R \geq \frac{Q^{2}}{M}$, see a detailed presentation ahead. 


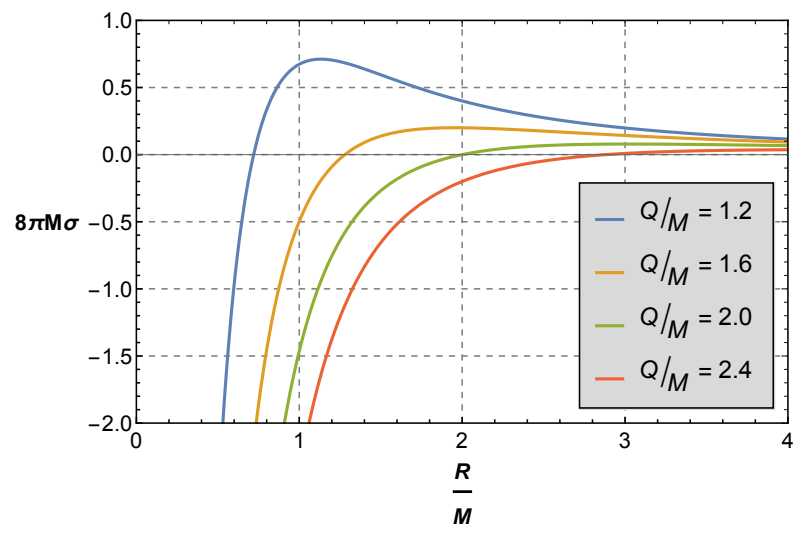

(a)

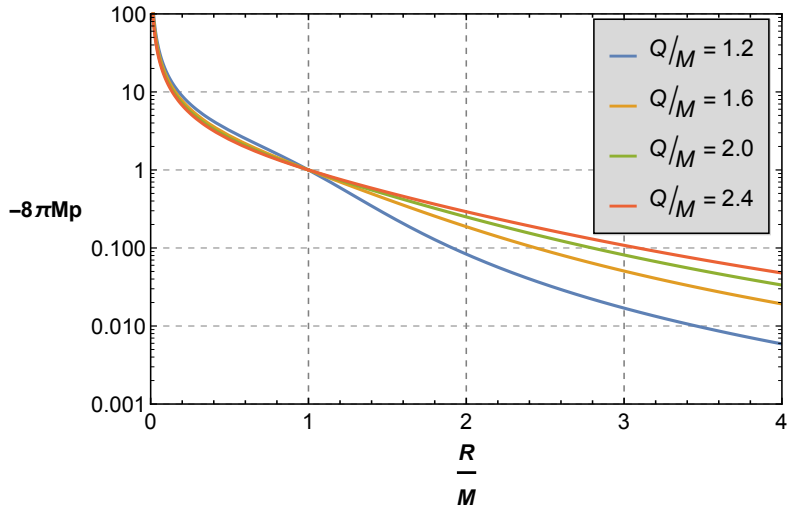

(b)

Figure 25: Physical properties of an overcharged star shell i.e., an electric perfect fluid thin shell in an overcharged

Reissner-Nordström state, in any location, i.e., $0<R<\infty$, and with orientation such that the normal points towards spatial infinity. Panel (a) Energy density $\sigma$ of the shell as a function of the radius $R$ of the shell for various values of the $\frac{Q}{M}$ ratio. The energy density is adimensionalized through the mass $M, 8 \pi M \sigma$, and the radius is adimensionalized through the mass $M, \frac{R}{M}$. Panel (b) Tension $-p$ on the shell as a function of the radius $R$ of the shell for various values of the $\frac{Q}{M}$ ratio. The tension is adimensionalized through the mass $M,-8 \pi M p$, and the radius is adimensionalized through the mass $M, \frac{R}{M}$.

The Carter-Penrose diagram can be drawn directly from the building blocks of an interior Minkowski spacetime and the exterior asymptotic infinite region of the overcharged Reissner-Nordström spacetime. In Figure 26 the CarterPenrose diagram of an overcharged Reissner-Nordström star shell spacetime for a junction surface with normal pointing towards spatial infinity is shown. It is clearly a star shell, a star in an asymptotically flat spacetime.

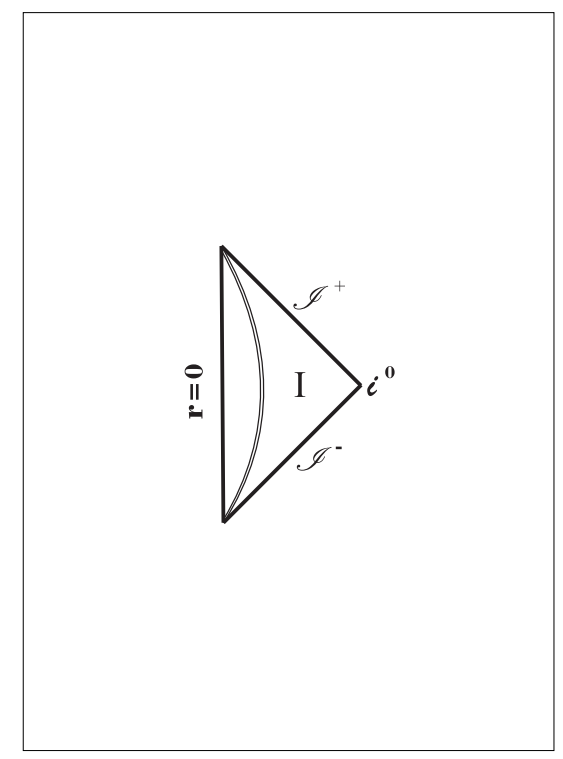

Figure 26: Carter-Penrose diagram of an overcharged star thin shell, i.e., a thin shell spacetime in an overcharged Reissner-Nordström state, with a thin shell located at any radius $R$, with orientation such that the normal points towards spatial infinity. The interior is Minkowski, the exterior is overcharged Reissner-Nordström. The shell is a star shell supported by tension and for sufficiently small $R$ also by negative energy density.

The physical interpretation of this case is clear cut, and it is similar to the corresponding nonextremal and extremal shells. This overcharged thin shell solution mimics an overcharged star. The energy density and pressure obey the energy conditions for certain radii. The causal and global structure as displayed by the Carter-Penrose diagram are 
well behaved and rather elementary. So, this case falls into the category of having the energy conditions verified and the geometrical setup is physically reasonable.

\section{B. Overcharged electric thin shells: Overcharged compact shell naked singularities}

Here we study the case of a fundamental electric thin shell in the overcharged state, i.e., $r_{+}$and $r_{-}$are not real, or $M<Q$, for which the shell's location is anywhere, i.e., $0<R<\infty$, and for which the orientation is such that the normal to the shell points towards $r=0$. In this case horizons do not exist and moreover $r_{+}$and $r_{-}$do not exist, and so there is neither gravitational radius nor Cauchy radius. The normal to the shell pointing towards $r=0$ means in the notation we have been using that $\xi=-1$, see the end of this section for details.

As functions of $M, Q$, and $R$, the shell's energy density $\sigma$ and pressure $p$, are, see the end of this section,

$$
\begin{gathered}
8 \pi \sigma=\frac{2}{R}(1+k), \\
8 \pi p=-\frac{1}{2 R k}\left[(1+k)^{2}-\frac{Q^{2}}{R^{2}}\right],
\end{gathered}
$$

respectively, with $k=\sqrt{1-\frac{2 M}{R}+\frac{Q^{2}}{R^{2}}}$. Also, the electric charge density $\sigma_{e}$ is given in terms of $M, Q$, and $R$, by Eq. (91). The behavior of $\sigma$ and $p$ as functions of the radial coordinate $R$ of the shell for various values of the $\frac{Q}{M}$ ratio in this case is shown in Figure 27. From Figure 27 we see that the energy density is positive for all shells. Also, this kind of thin shell is always supported by tension. It is a tension shell. The fact that it is supported by tension translates the well known fact that the Reissner-Nordström singularity at $r=0$ is repulsive. Moreover, we see that both the energy density and the tension of the shell diverge to infinity as the shell gets closer to $R=0$. On the other hand, in the limit of $R \rightarrow \infty$ both go to zero. An interesting feature of this kind of shells is the change in the behavior of $-p$ for $R>M$, where the tension needed to support such shells is smaller as the $\frac{Q}{M}$ ratio increases. Moreover, if the ratio $\frac{Q}{M}$ is in the range $1<\frac{Q}{M}<\sqrt{2}$ we find that the tension support of the matter fluid that composes this type of shells is an increasing function at $R=M$ and this function contains a local minimum in the region $0<R<M$. Notwithstanding, the minimum value is zero only in the extremal case, $\frac{Q}{M}=1$. When $Q=0$ there are no shells since we are not considering negative $M$. In relation to the energy conditions of the shell we can say that the null, the weak, and the dominant, energy conditions are verified when $R>0$, and the strong energy condition is verified when $R \leq \frac{Q^{2}}{M}$ in this case, see a detailed presentation ahead. 


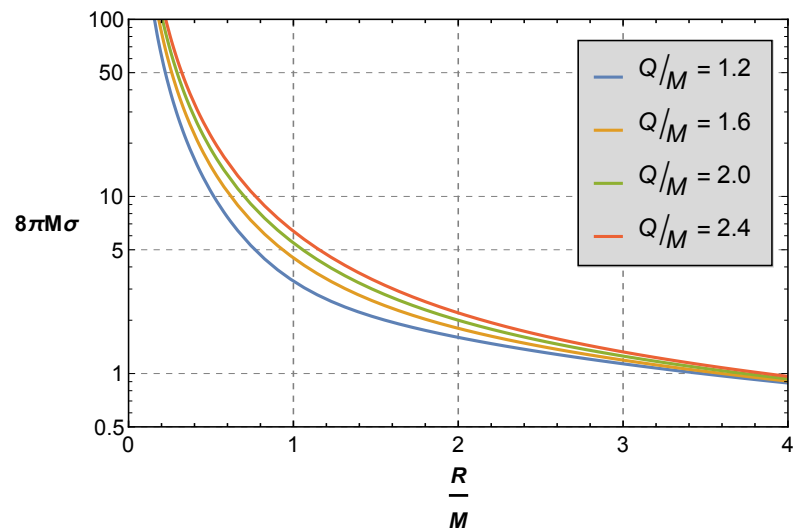

(a)

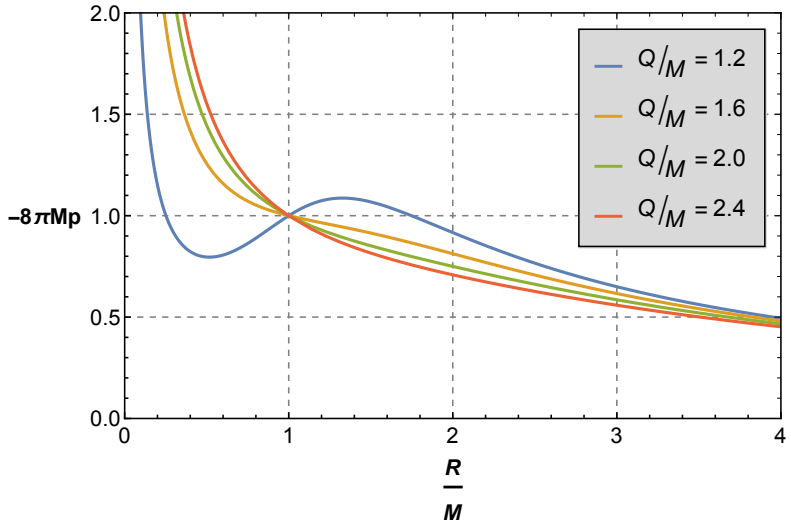

(b)

Figure 27: Physical properties of an overcharged compact shell naked singularity, i.e., an electric perfect fluid thin shell in an overcharged Reissner-Nordström state, in any location, i.e., $0<R<\infty$, and with orientation such that the normal points towards $r=0$. Panel (a) Energy density $\sigma$ of the shell as a function of the radius $R$ of the shell for various values of the $\frac{Q}{M}$ ratio. The energy density is adimensionalized through the mass $M, 8 \pi M \sigma$, and the radius is adimensionalized through the mass $M, \frac{R}{M}$. Panel (b) Tension $-p$ on the shell as a function of the radius $R$ of the shell for various values of the $\frac{Q}{M}$ ratio. The tension is adimensionalized through the mass $M,-8 \pi M p$, and the radius is adimensionalized through the mass $M, \frac{R}{M}$.

The Carter-Penrose diagram can be drawn directly from the building blocks of an interior Minkowski spacetime and the exterior region neighbor to $r=0$ of the overcharged Reissner-Nordström spacetime. In Figure 28 the Carter-Penrose diagram of an overcharged Reissner-Nordström star shell spacetime for a junction surface with normal pointing towards the $r=0$ singularity is shown. It is clearly a compact shell naked singularity, such that there is no clear distinction of what is outside from what is inside.

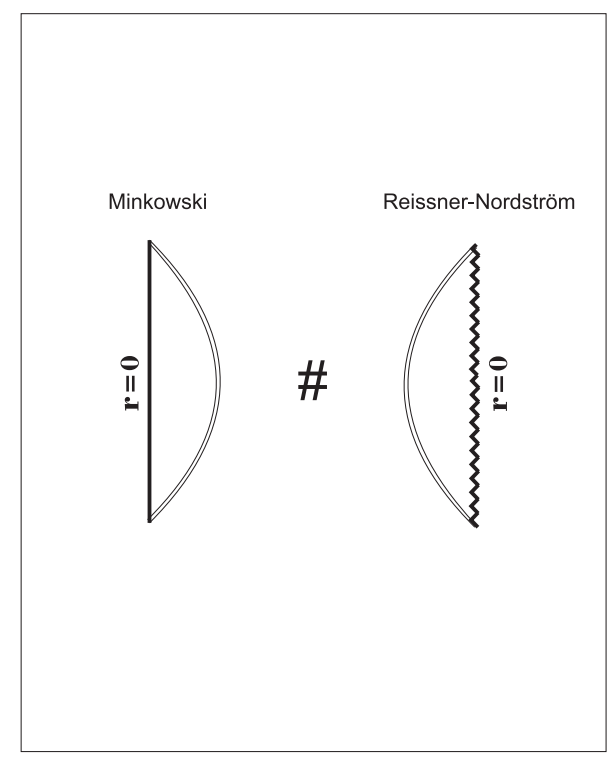

Figure 28: Carter-Penrose diagram of an overcharged compact shell naked singularity, i.e., a thin shell spacetime in an overcharged Reissner-Nordström state, located at any radius $R$, with orientation such that the normal points towards $r=0$. There is no clear distinction of what is outside from what is inside. The interior is Minkowski, the exterior is overcharged Reissner-Nordström. This is a compact shell naked singularity spacetime. 
The physical interpretation of this case is understood by now, it is similar to the corresponding nonextremal and extremal shells. This overcharged thin shell solution provides a closed spatial static universe with a singularity at one pole. There are no horizons. The energy density and pressure obey the energy conditions for certain shell radii. The causal and global structure as displayed by the Carter-Penrose diagram show the characteristics of this universe that has two sheets joined at the shell with one sheet having a singularity at its pole and with no horizons. The singularity is avoidable to timelike curves. So, this case falls into the category of having the energy conditions verified and the resulting spacetime being peculiar.

\section{Formalism for overcharged shells}

\section{Preliminaries}

We now make a careful study to derive the properties of the fundamental electric thin shell used in the two previous subsections, i.e., the thin shell in an overcharged state, i.e., $r_{+}$and $r_{-}$do not exist or $M<Q$, for which the shell's radius $R$ location obeys $0<R<\infty$, and for which the orientation is such that the normal to the shell points towards spatial infinity or towards $r=0$. It should be read as an appendix to the previous two subsections. We use the formalism developed in Sec. II.

\section{Induced metric, and extrinsic curvature of $\mathcal{S}$ as seen from $\mathcal{M}_{\mathrm{i}}$}

Let us start by analyzing the interior Minkowski spacetime, $\mathcal{M}_{\mathrm{i}}$. Since it is the same as the analysis done previously we only quote the important equations. They are the interior metric Eq. (28), the interior four-velocity of the shell Eq. (29), the metric for the shell at radius $R$ Eq. (30), the normal to the shell Eq. (31), and the extrinsic curvature from the inside Eq. (32).

\section{Induced metric, and extrinsic curvature of $\mathcal{S}$ as seen from $\mathcal{M}_{\mathrm{e}}$}

To proceed we have now to find the expressions for the induced metric on $\mathcal{S}$ and the extrinsic curvature components as seen from the exterior spacetime, $\mathcal{M}_{\mathrm{e}}$, in the overcharged state, i.e., $r_{+}$and $r_{-}$do not exist or $M<Q$, see Figure 3, for which the shell's obeys $0<R<\infty$, and for which the orientation is such that the normal to the shell points towards increasing $r$, i.e., towards spatial infinity, or towards decreasing $r$ i.e., towards $r=0$, as seen from the exterior, as used in the two previous subsections.

The line element for the overcharged Reissner-Nordström spacetime, now in the quantities $M$ and $Q$, since $r_{+}$and $r_{-}$do not exist, is

$$
d s_{\mathrm{e}}^{2}=-\left(1-\frac{2 M}{r}+\frac{Q^{2}}{r^{2}}\right) d t^{2}+\frac{d r^{2}}{1-\frac{2 M}{r}+\frac{Q^{2}}{r^{2}}}+r^{2} d \Omega^{2}
$$

where $M<Q$.

Considering a static shell as we have been doing, the components of the 4-velocity $u^{\alpha}$ of an observer comoving with the shell as seen from the exterior spacetime, are given by

$$
u_{\mathrm{e}}^{\alpha}=-\left(\frac{1}{k}, 0,0,0\right)
$$

where $k=\sqrt{1-\frac{2 M}{R}+\frac{Q^{2}}{R^{2}}}$. To find the induced metric on $\mathcal{S}$ as seen by an observer at $\mathcal{M}_{\mathrm{e}}$ and imposing the first junction condition, Eq. (8), we find that the shell's radial coordinate $R$ is the same as measured by an observer at $\mathcal{M}_{\mathrm{i}}$ or $\mathcal{M}_{\mathrm{e}}$ and the induced metric on $\mathcal{S}$ is given by Eq. (35), namely,

$$
\left.d s^{2}\right|_{\mathcal{S}}=-d \tau^{2}+R^{2} d \Omega^{2} .
$$

Combining $n_{\mathrm{e}}^{\alpha} n_{\mathrm{e} \alpha}=1$, see Eq. (3), $n_{\mathrm{e} \alpha} u_{\mathrm{e}}^{\alpha}=0$, see Eq. (4), and Eq. (95), we find the expression for the components of the unit normal to the hypersurface $\mathcal{S}$, as seen from the exterior spacetime $\mathcal{M}_{\mathrm{e}}$, to be $n_{\mathrm{e} \alpha}= \pm\left(0, \frac{1}{k}, 0,0\right)$. To specify the sign of the normal to $\mathcal{S}$ for each region we consider two orientations: the orientation where the normal $n$ 
points towards spatial infinity and the orientation where the normal points towards the singularity $r=0$. These two orientations can be treated in a concise way by using $\xi= \pm 1$, such that

$$
n_{\mathrm{e} \alpha}=\xi\left(0, \frac{1}{k}, 0,0\right) .
$$

Then, we find the nonzero components of the extrinsic curvature of $\mathcal{S}$ as seen from the exterior spacetime to be given by

$$
K_{\mathrm{e}}{ }^{\tau} \tau=\frac{\xi}{R^{2} k}\left(M-\frac{Q^{2}}{R}\right), \quad K_{\mathrm{e} \theta}{ }^{\theta}=K_{\mathrm{e}}^{\varphi} \varphi=\xi \frac{k}{R}
$$

where again $k$ is the redshift function given by $k=\sqrt{1-\frac{2 M}{R}+\frac{Q^{2}}{R^{2}}}$.

\section{Shell's energy density and pressure}

Having determined the components of the extrinsic curvature of the matching surface $\mathcal{S}$ as seen from the interior and exterior spacetimes we are now in position to use the second junction condition (9) to find the expressions for the energy density and pressure support of a perfect fluid thin shell in an overcharged state. Using the shell's stress-energy tensor given in Eq. (10) we find

$$
\begin{gathered}
8 \pi \sigma=\frac{2}{R}(1-\xi k), \\
8 \pi p=\frac{\xi}{2 R k}\left[(1-\xi k)^{2}-\frac{Q^{2}}{R^{2}}\right],
\end{gathered}
$$

where the redshift function of the shell at $r=R$ is given by $k=\sqrt{1-\frac{2 M}{R}+\frac{Q^{2}}{R^{2}}}$. Moreover, defining the surface electric current density $s_{a}$ on the thin shell as $s_{a}=\sigma_{e} u_{a}$, where $\sigma_{e}$ represents the electric charge density, and since the Minkowski spacetime has zero electric charge, from Eqs. (12)-(13) and (21) it follows that

$$
8 \pi \sigma_{e}=\frac{2 Q}{R^{2}}
$$

As before, we have to distinguish the two possible orientations provided by $\xi$. In Eqs. (99) and (100) it is clear that it is necessary to pick the sign in $\xi$. Let us start with $\xi=+1$. Eqs. (99) and (100) with $\xi=+1$ yield $8 \pi \sigma=\frac{2}{R}(1-k)$, $8 \pi p=\frac{1}{2 R k}\left[(1-k)^{2}-\frac{Q^{2}}{R^{2}}\right]$, and also from Eq. (101) we have $8 \pi \sigma_{e}=\frac{2 Q}{R^{2}}$. Let us now take $\xi=-1$. Eqs. (99) and (100) with $\xi=-1$ yield $8 \pi \sigma=\frac{2}{R}(1+k), 8 \pi p=-\frac{1}{2 R k}\left[(1+k)^{2}-\frac{Q^{2}}{R^{2}}\right]$, and also from Eq. (101) we have $8 \pi \sigma_{e}=\frac{2 Q}{R^{2}}$. These are the expressions used in the two previous subsections. Note that for the overcharged case $0<R<\infty$. 


\title{
IX. A SYNOPSIS TO ALL THE FUNDAMENTAL ELECTRIC THIN SHELLS: ENERGY CONDITIONS AND THE BEWILDERING VARIETY OF CARTER-PENROSE DIAGRAMS
}

\author{
A. Energy conditions for the fundamental electric thin shells
}

\section{Energy-conditions}

The analysis of the properties of the fundamental electric shells, i.e., timelike, static, perfect fluid thin shells with a Minkowski interior and a Reissner-Nordström exterior showed that both the energy density and pressure support depend on the state of the shell, on the location of the shell and on the orientation of the shell, i.e., on the direction of the outside pointing normal. Moreover, we saw that in some situations the energy density and pressure may take negative values, and this feature can also depend on the value of the radial coordinate of the shell. Here, we address the question of which shells and in what conditions do they verify the various energy conditions.

The energy conditions are a set of restrictions on the stress-energy tensor. In the case of a perfect fluid they lead to specific constraints on the energy density and pressure, see, e.g., [27] for energy conditions on shells, see also [14, 15] for energy conditions on shells and [41] for the original setting of energy conditions. Here we will study the null, weak, dominant, and strong energy conditions for the fundamental electric thin shells. Now, each energy condition may be considered to hold at any point of the spacetime or along a flowline, where the specific energy condition is only verified on average, allowing for pointwise violations. We consider the pointwise version of the energy conditions. Let us first briefly explain the physical motivation for each energy condition and their implications on the properties of a perfect fluid thin shell.

The null energy condition, or NEC, represents the restriction that the energy density of any matter distribution in spacetime experienced by a ligh-ray is nonnegative. For a generic stress-energy tensor $T_{\alpha \beta}$, this is represented by $T_{\alpha \beta} k^{\alpha} k^{\beta} \geq 0$ for any future pointing null vector field $k^{\alpha}$. For a perfect fluid thin shell with stress-energy tensor $S_{a b}$ given by Eq. (10) this implies

$$
\sigma+p \geq 0
$$

The weak energy condition, or WEC, is a more restrictive version of the NEC where it is imposed that the energy density of any matter distribution in spacetime measured by any timelike observer must be nonnegative, then $T_{\alpha \beta} v^{\alpha} v^{\beta} \geq 0$ for any future pointing, timelike vector field $v^{\alpha}$. For a perfect fluid thin shell with stress-energy tensor $S_{a b}$ given by Eq. (10) this leads to the following restrictions

$$
\sigma \geq 0, \quad \sigma+p \geq 0
$$

The dominant energy condition, or DEC, represents the statement that in addition to the WEC being verified, the flow of energy can never be observed to be faster than light, that is, in addition to $T_{\alpha \beta} v^{\alpha} v^{\beta} \geq 0$, the vector field $Y^{\alpha}$ with components given by $Y^{\alpha}=-T_{\beta}{ }^{\alpha} v^{\beta}$, verifies $Y^{\alpha} Y_{\alpha} \leq 0$, for any timelike future pointing vector field $v^{\alpha}$. For a perfect fluid thin shell with stress-energy tensor $S_{a b}$ given by Eq. (10) this implies

$$
\sigma \geq 0, \quad \sigma-|p| \geq 0
$$

The strong energy condition, or SEC, represents the restriction that nearby timelike geodesics are always focused towards each other, essentially guaranteeing that gravity is always perceived to be attractive by any timelike observer. In the case of general relativity, this is found by guaranteeing $\left(T_{\alpha \beta}-\frac{1}{2} g_{\alpha \beta} \mathcal{T}_{\gamma}^{\gamma}\right) v^{\alpha} v^{\beta} \geq 0$ for any timelike vector field $v^{\alpha}$. For a perfect fluid thin shell with stress-energy tensor $S_{a b}$ given by Eq. (10) we find,

$$
\sigma+p \geq 0, \quad \sigma+2 p \geq 0
$$

\section{Limiting radii from an analysis of the energy conditions on fundamental electric thin shells}

From Eqs. (102)-(105) we see that the energy conditions imply various restrictions on the energy density and pressure of a perfect fluid. In the considered setup, we have found that the properties of the perfect fluid fundamental electric thin shells are functions essentially of the radius $R$ of the shell. Hence, the constrains imposed by the energy conditions on the thin shell for the various possible spacetimes will lead to restrictions on $R$. Anticipating what follows, we present the expressions for the limiting radii $R_{\mathrm{I}}, R_{\mathrm{I}^{\prime}}$, and $R_{\mathrm{III}}$, that arise from solving the inequalities 
(102)-(105) for the various junction spacetimes, i.e.,

$$
\begin{aligned}
& R_{\mathrm{I}}=\frac{M}{36}\left[25+3\left(\frac{Q}{M}\right)^{2}+\frac{9\left(\frac{Q}{M}\right)^{4}-570\left(\frac{Q}{M}\right)^{2}+625}{\Delta_{\mathrm{I}}}+\Delta_{\mathrm{I}}\right], \\
& R_{\mathrm{I}^{\prime}}=\frac{M}{4}\left[3+\left(\frac{Q}{M}\right)^{2}+\frac{\left(\frac{Q}{M}\right)^{4}-10\left(\frac{Q}{M}\right)^{2}+9}{\Delta_{\mathrm{I}^{\prime}}}+\Delta_{\mathrm{I}^{\prime}}\right], \\
& R_{\mathrm{III}}=\frac{M}{72}\left[50+6\left(\frac{Q}{M}\right)^{2}-\frac{(1-i \sqrt{3})\left[9\left(\frac{Q}{M}\right)^{4}-570\left(\frac{Q}{M}\right)^{2}+625\right]}{\Delta_{\mathrm{I}}}-(1+i \sqrt{3}) \Delta_{\mathrm{I}}\right],
\end{aligned}
$$

with

$$
\begin{aligned}
\Delta_{\mathrm{I}} & =\sqrt[3]{27\left(\frac{Q}{M}\right)^{6}+216\left(\frac{Q}{M}\right)^{3} \sqrt{9\left(\frac{Q}{M}\right)^{4}+366\left(\frac{Q}{M}\right)^{2}-375}+5211\left(\frac{Q}{M}\right)^{4}-21375\left(\frac{Q}{M}\right)^{2}+15625} \\
\Delta_{\mathrm{I}^{\prime}} & =\sqrt[3]{8\left(\frac{Q}{M}\right)^{3} \sqrt{\left(\left(\frac{Q}{M}\right)^{2}-1\right)^{2}+\left(\frac{Q}{M}\right)^{6}+17\left(\frac{Q}{M}\right)^{4}-45\left(\frac{Q}{M}\right)^{2}+27}}
\end{aligned}
$$

The expressions for $R_{\mathrm{I}}$ and $R_{\mathrm{I}^{\prime}}$ can be read directly, the expression for $R_{\mathrm{III}}$ is written in terms of the imaginary unit $i$, but for the range of values of the ratio $\frac{Q}{M}$ of interest, this function takes purely real values. Moreover, although it is not clear from the expressions, the values of the radii $R_{\mathrm{I}}, R_{\mathrm{I}^{\prime}}$, and $R_{\mathrm{III}}$ are independent of the sign of $Q$, as expected. For completeness, in Figure 29 we present the behavior of the various limiting radii defined in Eqs. (106)-(108) as functions of the ratio $\frac{Q}{M}$.

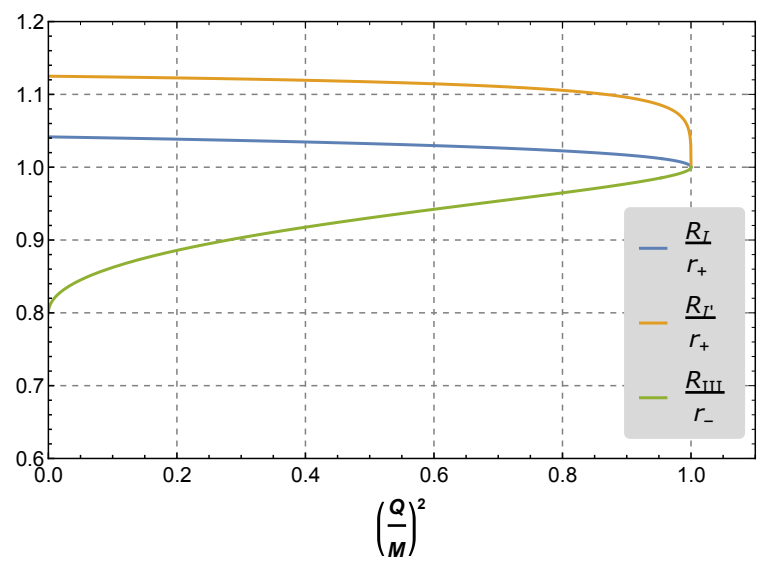

(a)

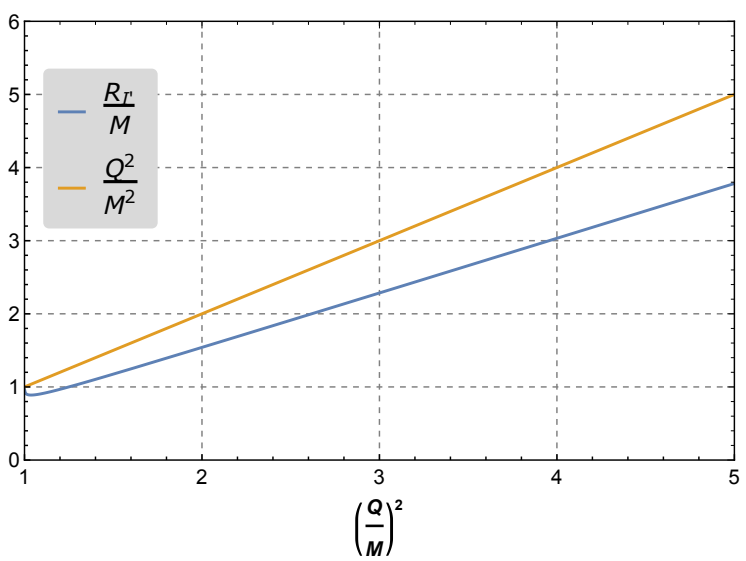

(b)

Figure 29: Behavior of the various radii, whose expressions are given by Eqs. (106) - (108), found by imposing the null, weak, dominant and strong energy conditions to the thin shells present at the matching surface of the various junction spacetimes. 


\section{Table of the energy conditions on fundamental electric thin shells}

Using the expressions for the energy density and pressure support for the thin matter shell for each resulting junction spacetime in the inequalities (102)-(105), allows us to find the constraints on the shell's location so that each of the tested energy conditions is verified. In the table of Figure 30 we summarize the results.

\begin{tabular}{|c|c|c|c|c|c|}
\hline & & $\begin{array}{c}\text { Null energy condition } \\
\text { NEC }\end{array}$ & $\begin{array}{c}\text { Weak energy condition } \\
\text { WEC }\end{array}$ & $\begin{array}{c}\text { Dominant energy condition } \\
\text { DEC }\end{array}$ & $\begin{array}{c}\text { Strong energy condition } \\
\text { SEC }\end{array}$ \\
\hline \multirow{4}{*}{ NonExtremal } & Section III A nonextremal outside $r_{+} \uparrow$ & $R>r_{+}$ & $R>r_{+}$ & $R \geq R_{\mathrm{I}}$ & $R>r_{+}$ \\
\hline & Section III B nonextremal outside $r_{+} \downarrow$ & $R \geq R_{\mathrm{I}^{\prime}}$ & $R \geq R_{\mathrm{I}^{\prime}}$ & $R \geq R_{\mathrm{I}^{\prime}}$ & Never verified \\
\hline & Section IV A nonextremal inside $r_{-} \uparrow$ & Never verified & Never verified & Never verified & Never verified \\
\hline & Section IV B nonextremal inside $r_{-} \downarrow$ & $0<R<r_{-}$ & $0<R<r_{-}$ & $0<R \leq R_{\mathrm{III}}$ & $0<R<r_{-}$ \\
\hline \multirow{8}{*}{ Extremal } & Section VA extremal outside $r_{+} \uparrow$ & $R>r_{+}$ & $R>r_{+}$ & $R>r_{+}$ & $R>r_{+}$ \\
\hline & Section VB extremal outside $r_{+} \downarrow$ & $R>r_{+}$ & $R>r_{+}$ & $R>r_{+}$ & Never verified \\
\hline & Section VI A extremal inside $r_{+} \uparrow$ & Never verified & Never verified & Never verified & Never verified \\
\hline & Section VIB extremal inside $r_{+} \downarrow$ & $0<R<r_{+}$ & $0<R<r_{+}$ & $0<R<r_{+}$ & $0<R<r_{+}$ \\
\hline & Section VII A extremal at $r_{+} \uparrow>$ & Always verified & Always verified & Always verified & Always verified \\
\hline & Section VII A extremal at $r_{+} \downarrow>$ & Always verified & Always verified & Always verified & Never verified \\
\hline & Section VII B extremal at $r_{+} \uparrow<$ & Always verified & Always verified & Always verified & Never verified \\
\hline & Section VII B extremal at $r_{+} \downarrow<$ & Always verified & Always verified & Always verified & Always verified \\
\hline \multirow{2}{*}{ Overcharged } & Section VIII A overcharged $\uparrow$ & $R \geq R_{\mathrm{I}^{\prime}}$ & $R \geq R_{\mathrm{I}^{\prime}}$ & $R \geq R_{\mathrm{I}^{\prime}}$ & $R \geq \frac{Q^{2}}{M}$ \\
\hline & Section VIII B overcharged $\downarrow$ & $R>0$ & $R>0$ & $R>0$ & $R \leq \frac{Q^{2}}{M}$ \\
\hline
\end{tabular}

Figure 30: Range of values of the radius $R$ of the fundamental electric thin shell, in the various allowed locations of the exterior Reissner-Nordström spacetime for which, the null, weak, dominant and strong energy conditions are verified. The symbols $\uparrow$ and $\downarrow$ denote the orientation of the shell, i.e., outward normal pointing to increasing radius and to decreasing radius from the shell, respectively. The symbols $>$ and $<$ for Sections VII A and VIIB in the table denote whether the approach to $r_{+}$is done through $R>r_{+}$or $R<r_{+}$, respectively.

\section{Detailed description}

For the fundamental electric shells in a nonextremal state, located outside the gravitational radius $r_{+}, R>r_{+}$, we find that when their orientation is such that the outward normal points to spatial infinity, Section III A, i.e., the star shells, they always verify the NEC and WEC, they verify the DEC for $R>R_{\mathrm{I}}$, and also always verify the SEC, and when their orientation is such that the outward normal points to the gravitational radius $r_{+}$, Section IIIB, i.e., the tension shell black holes, they verify the NEC, WEC, and DEC for $R>R_{\mathrm{I}^{\prime}}$, and the SEC is always violated. Moreover, the limiting radius $R_{\mathrm{I}^{\prime}}$ of Eq. (107) also determines the value of the circumferential radius of the shell for which its energy density is maximum and thus it is connected to the bumps in de energy density $\sigma$ of Figure 7 . For the fundamental electric shells in a nonextremal state, located inside the Cauchy radius $r_{-}, R<r_{-}$, we find that when their orientation is such that the outward normal points to $r_{-}$, Section IV A, the tension shell regular and nonregular black holes, none of the energy conditions are verified, and when their orientation is such that the outward normal points to the $r=0$ singularity, Section IV B, the compact shell naked singularities, the shells always verify the NEC and WEC, verify the DEC in the domain $0<R \leq R_{\mathrm{III}}$, and always verify the SEC.

For the fundamental electric shells in an extremal state, $r_{+}=r_{-}$, located outside the gravitational radius $r_{+}$, $R>r_{+}$, we find that when their orientation is such that the outward normal points to spatial infinity, Section $\mathrm{V}$ A, i.e., the Majumdar-Papapetrou star shells, they always verify the NEC, WEC, DEC, and SEC, and when their orientation is such that the outward normal points to the event horizon, Section VB, the extremal tension shell black holes, they always verify the NEC, WEC, and DEC, and the SEC is always violated. For the fundamental electric shells in an extremal state, $r_{+}=r_{-}$, located inside the gravitational radius, $R<r_{+}$, we find that when their orientation is such that the outward normal points to spatial infinity, Section VI A, the extremal tension shell regular and nonregular black holes, none of the energy conditions are verified by the shells, and when their orientation is such that the outward normal points to the $r=0$ singularity, Section VIB, the Majumdar-Papapetrou compact shell naked singularities, the shells always verify the NEC, WEC, DEC, and SEC. For the fundamental electric shells in an 
extremal state, $r_{+}=r_{-}$, located in the limit at the gravitational radius, $R=r_{+}$, we find that when their orientation is such that the outward normal points to spatial infinity and the limit of $R \rightarrow r_{+}$comes from above, Section VII A 1 , the Majumdar-Papapetrou shell quasiblack holes, the shells always verify the NEC, WEC, DEC, and SEC, the matter is Majumdar-Papapetrou matter, whereas when the limit of $R \rightarrow r_{+}$comes from below, Section VIIA 2, the extremal null shell black holes, the shells verify the NEC, WEC, DEC, and never verify the SEC, and when their orientation is such that the outward normal points to the $r=0$ singularity and the limit of $R \rightarrow r_{+}$comes from above, Section VIIB 1, the extremal tension shell null singularities, one has that these shells always verify the NEC, WEC and DEC, and never verify the SEC, whereas when the limit of $R \rightarrow r_{+}$comes from below, Section VII B 2, the extremal Majumdar-Papapetrou null shell singularities, the shells verify the NEC, WEC, DEC, and SEC, the matter is Majumdar-Papapetrou matter.

For the fundamental electric shells in an overcharged state, $r_{+}$and $r_{-}$do not exist and $M<Q$, located at any radius $R$, we find that when their orientation is such that the outward normal points to spatial infinity, Section VIII A, the overcharged star shells, the shells verify the NEC, WEC, DEC for $R \geq R_{\mathrm{I}^{\prime}}$, and the SEC when $R \geq \frac{Q^{2}}{M}$, and when their orientation is such that the outward normal points to the $r=0$ singularity, Section VIIIB, the overcharged compact shell naked singularities, the NEC, WEC, DEC are always satisfied and the SEC when $R \leq \frac{Q^{2}}{M}$. The results for the strong energy condition of an overcharged shell indicate that in the overcharged Reissner-Nordström spacetime the singularity is repulsive in a core region within $r<\frac{Q^{2}}{M}$. Our result extends that of [38, 40] where it was found that the nonextremal and extremal Reissner-Nordström solutions are characterized by a repulsive region delimited, respectively, by the Cauchy or event horizons. Here, although there are no horizons, we see that the same conclusion holds, and confirm the result given in, e.g., [43] that there is a repulsive region in the overcharged Reissner-Nordström spacetime near the singularity, and, in addition, find the limiting radius of this repulsive region. 


\section{B. The bewildering variety of the Carter-Penrose diagrams for the fundamental electric thin shells}

In addition to performing an analysis on the physical properties of the shells, i.e., their energy density $\sigma$, pressure $p$, and the corresponding energy conditions, we have drawn the Carter-Penrose diagram in each of the fourteen cases. These diagrams for the fundamental electric thin shells are summarized in the chart of Figure 31 which displays clearly their bewildering variety. There were cases that the solution does not tell precisely how to continue the Carter-Penrose

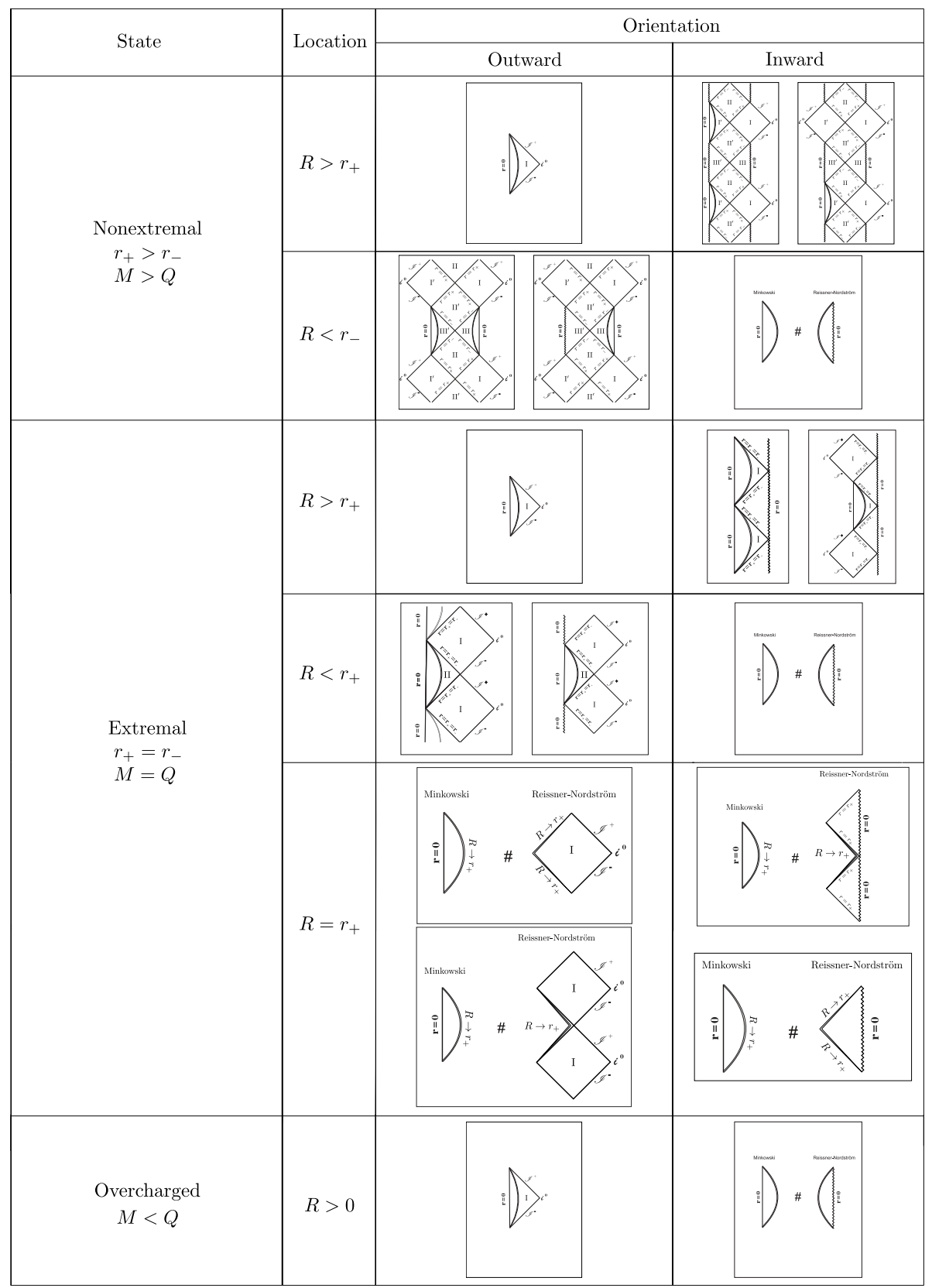

Figure 31: A chart with all the fourteen different Carter-Penrose diagrams for the fundamental electric charged shells, i.e., static shells with a Minkowski interior and a Reissner-Nordström exterior.

diagram, one can either repeat the shell, or draw horizons and infinities at will, in any possible combination. 


\section{CONCLUSIONS}

We have classified and studied the spacetimes generated by a fundamental electric thin shell, i.e., a spherical static electrical thin shell with a Minkowski interior and a Reissner-Nordström exterior. All three main states a shell with a Reissner-Nordström exterior can have were considered, namely, nonextremal, extremal, and overcharged. In the nonextremal state there are still two possible locations for the shell, namely, the shell is located outside the gravitational radius or the shell is located inside the Cauchy radius. In the extremal state there are three possibilities, namely, the shell is located outside the gravitational radius, the shell is located inside the gravitational radius, or the shell is located at the gravitational radius. In the overcharged state there is only one possibility, the shell can be located anywhere. We have seen, in the wake of the work of Lynden-Bell and Katz for non-electrical thin shells with a Schwarzschild exterior, that each of the locations has still two possibilities, either the outward normal to the shell points toward increasing radius or it points toward decreasing radius. For extremal shells at the gravitational radius there is still a subdivision, either the shell approaches the gravitational radius from above, or it approaches the gravitational radius from below. In all there are fourteen different cases.

For each of the fourteen different shells we have worked out the energy density $\sigma$ and the pressure $p$ and analyzed the energy conditions of the matter on the shell. In addition we have drawn the Carter-Penrose diagrams in all the fourteen cases. There were cases that the solution does not tell precisely how to continue the diagram, one can either repeat the shell, or draw horizons and infinities at will, in any possible combination. In addition, in some cases the distinction between what is interior and what is exterior is blurred. The maximum analytical extension of the fundamental electric shells and consequent Carter-Penrose diagrams, showed that there is a plethora of solutions that encompass nonextremal star shells, nonextremal tension shell black holes, nonextremal tension shell regular and nonregular black holes, nonextremal compact shell naked singularities, Majumdar-Papapetrou star shells, extremal tension shell singularities, extremal tension shell regular and nonregular black holes, Majumdar-Papapetrou compact shell naked singularities, Majumdar-Papapetrou shell quasiblack holes, extremal null shell quasinonblack holes, extremal null shell singularities, Majumdar-Papapetrou null shell singularities, overcharged star shells, and overcharged compact shell naked singularities.

In some of the cases it was found that the energy conditions are verified and the geometrical setup is physically reasonable, in other cases it was found that the energy conditions are verified but the resulting geometry is rather peculiar, or even strange, or that the energy conditions are violated but the resulting geometry seems physically reasonable. Therefore, the set of solutions might be greatly reduced if we only choose solutions which indeed obey the energy conditions and are physically reasonable or, regard only solutions that verify the energy conditions, independently of the geometrical setup, or, maintain only solutions whose geometry seems reasonable. Here we choose to maintain everything as good and interesting solutions, to be tested a posteriori.

\section{ACKNOWLEDGMENTS}

JPSL acknowledges Fundação para a Ciência e Tecnologia - FCT, Portugal, for financial support through Project No. UIDB/00099/2020. PL acknowledges IDPASC and FCT, Portugal, for financial support through Grant No. PD/BD/114074/2015, and thanks Centro de Matemática, Universidade do Minho, where part of this work has been performed, for the hospitality. 


\section{Appendix A: Kruskal-Szekeres coordinates of the nonextremal Reissner-Nordström spacetime}

\section{General formalism for a static spherically symmetric spacetime}

In this appendix we will construct two coordinate systems for the nonextremal Reissner-Nordström spacetime, one well behaved in a neighborhood of the event horizon, $r=r_{+}$, and the other in a neighborhood of the Cauchy horizon, $r=r_{-}$, which, together, cover the full nonextremal Reissner-Nordström spacetime. To find these new coordinate systems we will use the formalism introduced in [38] which we now present briefly.

Given a static, spherically symmetric spacetime whose line element can be written in the form

$$
d s^{2}=-\Phi(r) d t^{2}+\Phi^{-1}(r) d r^{2}+r^{2} d \Omega^{2}
$$

where the function $\Phi(r)$ is assumed to have zeros or poles representing coordinate singularities, which can be removed by a change of coordinates. Let us determine a simultaneous transformation of the coordinates $r$ and $t$ to new coordinates $X(r, t)$ and $T(r, t)$ such that the line element can be written as

$$
d s^{2}=f^{2}(X, T)\left(d X^{2}-d T^{2}\right)+r^{2}(X, T) d \Omega^{2},
$$

where $f^{2}(X, T)$ is to be regular in a sub-region covered by the coordinates $X$ and $T$. Comparing Eqs.(A1) and (A2) it is found that [38]

$$
\begin{aligned}
& X=h\left(r^{*}+t\right)+g\left(r^{*}-t\right), \\
& T=h\left(r^{*}+t\right)-g\left(r^{*}-t\right),
\end{aligned}
$$

with $d r^{*}=\Phi^{-1}(r) d r$, i.e.,

$$
r^{*}=\int \Phi^{-1}(r) d r
$$

$h$ and $g$ are arbitrary functions of one variable, and

$$
f^{2}=\frac{\Phi(r)}{4 h^{\prime}\left(r^{*}+t\right) g^{\prime}\left(r^{*}-t\right)},
$$

where prime denotes differentiation with respect to the functions variable, and $r^{*}=r^{*}(r)$ as given in Eq. (A4). In order to $f^{2}$ given in Eq. (A5) be non singular, any singularity in the numerator $\Phi(r)$ must be canceled by the denominator, for all $t$. Assuming $\Phi$ to have only poles of order 1 , and setting

$$
\begin{aligned}
& h\left(r^{*}+t\right)=A e^{\gamma\left(r^{*}+t\right)}, \\
& g\left(r^{*}-t\right)=B e^{\gamma\left(r^{*}-t\right)},
\end{aligned}
$$

where the scale factors $A$ and $B$ are complex numbers, it is possible to choose a value for the constant $\gamma$ such that $f^{2}$ is regular and positive throughout the region covered by the coordinate patch. Substituting Eq. (A6) into Eqs. (A3) and (A5) we find

$$
f^{2}=\frac{\Phi(r) e^{-2 \gamma r^{*}}}{4 A B \gamma^{2}}
$$

and

$$
\begin{aligned}
& X(r, t)=A e^{\gamma\left(r^{*}+t\right)}+B e^{\gamma\left(r^{*}-t\right)}, \\
& T(r, t)=A e^{\gamma\left(r^{*}+t\right)}-B e^{\gamma\left(r^{*}-t\right)},
\end{aligned}
$$

in terms of the coordinates $r$ and $t$. From Eq. (A8) we can find the inverse transformation and define the coordinate $r$, implicitly, in terms of the coordinates $X$ and $T$, such that

$$
X^{2}-T^{2}=4 A B e^{2 \gamma r^{*}} \text {. }
$$

Lastly, since $f^{2}$ in Eq. (A7) depends on the values of $A$ and $B, A$ and $B$ themselves have to be chosen in such a way that $f^{2}$ is positive. Moreover, given that the transformation between the coordinates $\{r, t\}$ and $\{X, T\}$ depends on $A$ and $B$, these must be chosen such that the coordinates $X$ and $T$ take only real values. 


\section{The general formalism applied specifically to the nonextremal Reissner-Nordström spacetime}

\section{a. The Reissner-Nordström spacetime}

Having introduced the general formalism, we can apply it specifically to the the nonextremal Reissner-Nordström spacetime. For this spacetime, the line element in terms of the coordinates $\{r, t\}$ is given by Eq. (A1) with

$$
\Phi(r)=\frac{\left(r-r_{+}\right)\left(r-r_{-}\right)}{r^{2}},
$$

where $r_{+}$is the gravitational or event horizon radius, and $r_{-}$is the Cauchy horizon radius. In terms of the mass $M$ and the electric charge $Q, r_{+}$and $r_{-}$are given by

$$
r_{ \pm}=M \pm \sqrt{M^{2}-Q^{2}}
$$

such that $\Phi(r)$ in Eq. (A10) can also be written as $\Phi(r)=1-\frac{2 M}{r}+\frac{Q^{2}}{r^{2}}$. Inverting Eq. (A11) one has $2 M=r_{+}+r_{-}$ and $Q=\sqrt{r_{+} r_{-}}$. From Eq. (A10), in these coordinates we see that the line element for the nonextremal ReissnerNordström spacetime, i.e., $\left(M^{2}>Q^{2}\right)$ contains two coordinate singularities at $r=r_{+}$and at $r=r_{-}$. Then, using the formalism of subsection A 1, two coordinate patches need to be found, each well defined in the neighborhood of each of the coordinate singularities. Notice, however, that there is a common region where both coordinate patches overlap.

\section{b. Removal of the coordinate singularity at the event horizon $r_{+}$}

Let us first find a coordinate patch that covers a neighborhood of the coordinate singularity at $r=r_{+}$. Using Eq. (A4), for the nonextremal Reissner-Nordström spacetime, $r^{*}$ is given by

$$
r^{*}=r+\frac{r_{+}^{2}}{r_{+}-r_{-}} \log \left(\frac{r-r_{+}}{r_{+}+r_{-}}\right)-\frac{r_{-}^{2}}{r_{+}-r_{-}} \log \left(\frac{r-r_{-}}{r_{+}+r_{-}}\right),
$$

where we have set the value of the integration constant to $r_{+}+r_{-}$. To remove the coordinate singularity at $r=r_{+}$ we will impose the constant $\gamma$ that appears in Eqs. (A6)-(A9) to take the following value

$$
\gamma=\frac{r_{+}-r_{-}}{2 r_{+}^{2}}
$$

Substituting Eqs. (A12) and (A13) into Eq. (A7) we find

$$
f^{2}=\frac{\left(r_{+}+r_{-}\right)^{2}}{A B r^{2}}\left(\frac{r_{+}^{2}}{r_{+}-r_{-}}\right)^{2} e^{-\frac{r\left(r_{+}-r_{-}\right)}{r_{+}^{2}}}\left(\frac{r-r_{-}}{r_{+}+r_{-}}\right)^{1+\left(\frac{r_{-}}{r_{+}}\right)^{2}},
$$

which is well behaved near the event horizon at $r=r_{+}$. As was stated in the previous section, the choice of the scale-factors $A$ and $B$ is quite arbitrary and we impose their values to be such that in the limit when the electric charge $Q$ goes to zero we recover the Kruskal-Szekeres coordinates defined in [42] for the Schwarzschild spacetime. So, the values for the scale-factors for the various regions are

$$
\begin{gathered}
\mathrm{I}\left\{\begin{array} { l l } 
{ A = } & { \frac { 1 } { 2 } } \\
{ B = } & { \frac { 1 } { 2 } , }
\end{array} \quad \mathrm { I } ^ { \prime } \left\{\begin{array}{l}
A=-\frac{1}{2} \\
B=-\frac{1}{2},
\end{array}\right.\right. \\
\mathrm{II}\left\{\begin{array}{ll}
A=-\frac{i}{2} \\
B= & \frac{i}{2}
\end{array},\right.
\end{gathered}
$$

We see that our choice for the scale-factors differs for each region. This is a consequence of the behavior of the coordinates $\{r, t\}$. Nonetheless, obviously, the geometry of the spacetime is unaltered since different choices for the scale factor that obey the restrictions imposed in the previous section give the same expression for the metric, aside a conformal constant factor. Our choice, though, leaves the metric completely unaltered, hence, substituting the various values for the scale-factors listed in Eq. (A15) into Eq. (A14) we get, for every region covered by the coordinate patch,

$$
f^{2}=\frac{4\left(r_{+}+r_{-}\right)^{2}}{r^{2}}\left(\frac{r_{+}^{2}}{r_{+}-r_{-}}\right)^{2} e^{-\frac{r\left(r_{+}-r_{-}\right)}{r_{+}^{2}}}\left(\frac{r-r_{-}}{r_{+}+r_{-}}\right)^{1+\left(\frac{r_{-}}{r_{+}}\right)^{2}} .
$$


Substituting Eq. (A15) into Eq. (A9) allow us to write the inverse transformation for the coordinate $r$ in terms of the coordinates $X$ and $T$ as

$$
X^{2}-T^{2}=e^{\frac{r\left(r_{+}-r_{-}\right)}{r_{+}^{2}}}\left(\frac{r-r_{+}}{r_{+}+r_{-}}\right)\left(\frac{r-r_{-}}{r_{+}+r_{-}}\right)^{-\left(\frac{r_{-}}{r_{+}}\right)^{2}} .
$$

For completeness, we also define the transformations for the coordinates $\{T, X\}$ in terms of the coordinates $\{r, t\}$ for the various regions. Substituting Eqs. (A13) and (A15) into Eq. (A8) we find

$$
\begin{aligned}
& \mathrm{I}\left\{\begin{array}{l}
X=e^{\frac{r\left(r_{+}-r_{-}\right)}{2 r_{+}^{2}}}\left(\frac{r-r_{+}}{r_{+}+r_{-}}\right)^{\frac{1}{2}}\left(\frac{r-r_{-}}{r_{+}+r_{-}}\right)^{-\frac{r_{-}^{2}}{2 r_{+}^{2}}} \cosh \left(\frac{t\left(r_{+}-r_{-}\right)}{2 r_{+}^{2}}\right), \\
T=e^{\frac{r\left(r_{+}-r_{-}\right)}{2 r_{+}^{2}}}\left(\frac{r-r_{+}}{r_{+}+r_{-}}\right)^{\frac{1}{2}}\left(\frac{r-r_{-}}{r_{+}+r_{-}}\right)^{-\frac{r_{-}^{2}}{2 r_{+}^{2}}} \sinh \left(\frac{t\left(r_{+}-r_{-}\right)}{2 r_{+}^{2}}\right)
\end{array}\right. \\
& \mathrm{I}^{\prime}\left\{\begin{array}{l}
X=-e^{\frac{r\left(r_{+}-r_{-}\right)}{2 r_{+}^{2}}}\left(\frac{r-r_{+}}{r_{+}+r_{-}}\right)^{\frac{1}{2}}\left(\frac{r-r_{-}}{r_{+}+r_{-}}\right)^{-\frac{r_{-}^{2}}{2 r_{+}^{2}}} \cosh \left(\frac{t\left(r_{+}-r_{-}\right)}{2 r_{+}^{2}}\right), \\
T=-e^{\frac{r\left(r_{+}-r_{-}\right)}{2 r_{+}^{2}}}\left(\frac{r-r_{+}}{r_{+}+r_{-}}\right)^{\frac{1}{2}}\left(\frac{r-r_{-}}{r_{+}+r_{-}}\right)^{-\frac{r_{-}^{2}}{2 r_{+}^{2}}} \sinh \left(\frac{t\left(r_{+}-r_{-}\right)}{2 r_{+}^{2}}\right)
\end{array},\right. \\
& \text { II }\left\{\begin{array}{l}
X=e^{\frac{r\left(r_{+}-r_{-}\right)}{2 r_{+}^{2}}}\left(\frac{r_{+}-r}{r_{+}+r_{-}}\right)^{\frac{1}{2}}\left(\frac{r-r_{-}}{r_{+}+r_{-}}\right)^{-\frac{r_{-}^{2}}{2 r_{+}^{2}}} \sinh \left(\frac{t\left(r_{+}-r_{-}\right)}{2 r_{+}^{2}}\right) \\
T=e^{\frac{r\left(r_{+}-r_{-}\right)}{2 r_{+}^{2}}}\left(\frac{r_{+}-r}{r_{+}+r_{-}}\right)^{\frac{1}{2}}\left(\frac{r-r_{-}}{r_{+}+r_{-}}\right)^{-\frac{r_{-}^{2}}{2 r_{+}^{2}}} \cosh \left(\frac{t\left(r_{+}-r_{-}\right)}{2 r_{+}^{2}}\right)
\end{array},\right. \\
& \mathrm{II}^{\prime}\left\{\begin{array}{l}
X=-e^{\frac{r\left(r_{+}-r_{-}\right)}{2 r_{+}^{2}}}\left(\frac{r_{+}-r}{r_{+}+r_{-}}\right)^{\frac{1}{2}}\left(\frac{r-r_{-}}{r_{+}+r_{-}}\right)^{-\frac{r_{-}^{2}}{2 r_{+}^{2}}} \sinh \left(\frac{t\left(r_{+}-r_{-}\right)}{2 r_{+}^{2}}\right) \\
T=-e^{\frac{r\left(r_{+}-r_{-}\right)}{2 r_{+}^{2}}}\left(\frac{r_{+}-r}{r_{+}+r_{-}}\right)^{\frac{1}{2}}\left(\frac{r-r_{-}}{r_{+}+r_{-}}\right)^{-\frac{r_{-}^{2}}{2 r_{+}^{2}}} \cosh \left(\frac{t\left(r_{+}-r_{-}\right)}{2 r_{+}^{2}}\right)
\end{array} .\right.
\end{aligned}
$$

This relations for $X$ and $T$ can be used to find the coordinate $t$ as a function of these coordinates, such that

$$
\begin{array}{ll}
t=\frac{2 r_{+}^{2}}{r_{+}-r_{-}} \operatorname{arctanh}\left(\frac{T}{X}\right) \quad, \text { in regions I and } \mathrm{I}^{\prime}, \\
t=\frac{2 r_{+}^{2}}{r_{+}-r_{-}} \operatorname{arctanh}\left(\frac{X}{T}\right) & , \text { in regions II and II'. }
\end{array}
$$

The spacetime diagram for this coordinate patch together with the relevant coordinate transformations are exhibited graphically in Figure A1. We further note that in-between region I and region I' there is an Einstein-Rosen bridge, i.e., a nontraversable dynamical wormhole. 


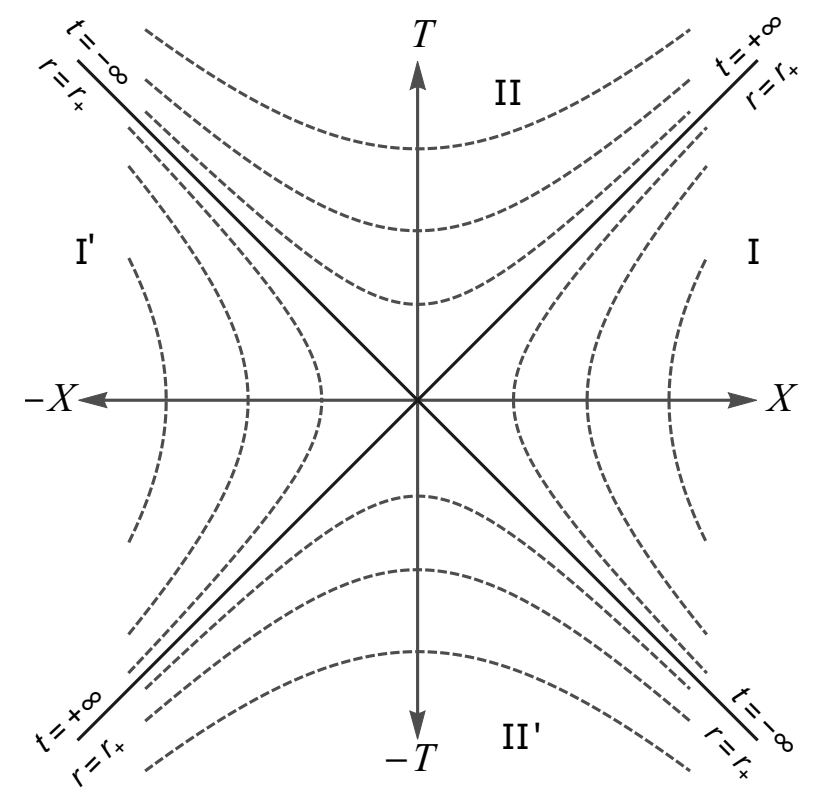

Figure A1: Relation between the Kruskal-Szekeres coordinates $\{X, T\}$ that cover a neighborhood of the event horizon and the Schwarzschild coordinates $\{r, t\}$. The hyperbolas represent curves of constant $r$ coordinate while curves of constant $t$ are straight lines through the origin.

c. Removal of the coordinate singularity at the Cauchy horizon $r_{-}$

Let us now define a second coordinate patch where the coordinate singularity at the Cauchy horizon, $r=r_{-}$, is removed. Here the function $r^{*}$ is now given by

$$
r^{*}=r+\frac{r_{+}^{2}}{r_{+}-r_{-}} \log \left(\frac{r_{+}-r}{r_{+}+r_{-}}\right)-\frac{r_{-}^{2}}{r_{+}-r_{-}} \log \left(\frac{r_{-}-r}{r_{+}+r_{-}}\right) .
$$

This change in the expression for the function $r^{*}$, compared with Eq. (A12), should not come with surprise since, as was stated in the previous subsection, the function $r^{*}$ is defined up to an integration constant which will be chosen such that the metric is real for each coordinate patch.

Now, since we want to remove the coordinate singularity at $r=r_{-}$, we impose

$$
\gamma=-\frac{r_{+}-r_{-}}{2 r_{-}^{2}} .
$$

Substituting Eqs. (A20) and (A21) into Eq. (A7) we find

$$
f^{2}=\frac{\left(r_{+}+r_{-}\right)^{2}}{A B r^{2}}\left(\frac{r_{-}^{2}}{r_{+}-r_{-}}\right)^{2} e^{\frac{r\left(r_{+}-r_{-}\right)}{r_{-}^{2}}}\left(\frac{r_{+}-r}{r_{+}+r_{-}}\right)^{1+\left(\frac{r_{+}}{r_{-}}\right)^{2}} .
$$

As in the previous subsection, we have now to choose the scale-factors $A$ and $B$ for the various sub-regions covered by the second coordinate patch hence,

$$
\begin{aligned}
& \text { III }\left\{\begin{array} { l l } 
{ A = } & { \frac { 1 } { 2 } } \\
{ B = } & { \frac { 1 } { 2 } , }
\end{array} \quad \text { III' } ^ { \prime } \left\{\begin{array}{l}
A=-\frac{1}{2} \\
B=-\frac{1}{2}
\end{array},\right.\right. \\
& \text { II }\left\{\begin{array} { l r } 
{ A = } & { \frac { i } { 2 } } \\
{ B = } & { - \frac { i } { 2 } }
\end{array} , \quad \text { II } ^ { \prime } \quad \left\{\begin{array}{lr}
A= & -\frac{i}{2} \\
B= & \frac{i}{2}
\end{array}\right.\right. \text {. }
\end{aligned}
$$

This choice for the scale factors leaves the expression for the metric unaltered for the various sub-regions covered by 
the coordinate patch, such that,

$$
f^{2}=\frac{4\left(r_{+}+r_{-}\right)^{2}}{r^{2}}\left(\frac{r_{-}^{2}}{r_{+}-r_{-}}\right)^{2} e^{\frac{r\left(r_{+}-r_{-}\right)}{r_{-}^{2}}}\left(\frac{r_{+}-r}{r_{+}+r_{-}}\right)^{1+\left(\frac{r_{+}}{r_{-}}\right)^{2}} .
$$

Substituting Eq. (A24) in Eq. (A9) we find

$$
X^{2}-T^{2}=e^{-\frac{r\left(r_{+}-r_{-}\right)}{r_{-}^{2}}}\left(\frac{r_{-}-r}{r_{+}+r_{-}}\right)\left(\frac{r_{+}-r}{r_{+}+r_{-}}\right)^{-\left(\frac{r_{+}}{r_{-}}\right)^{2}},
$$

which defines, implicitly, the coordinate $r$ in terms of the coordinates $X$ and $T$. For completeness we define the transformations for the various sub-regions covered by the coordinate patch that relate the coordinates $\{X, T\}$ with the coordinates $\{r, t\}$. Substituting Eqs. (A21) and (A23) in Eq. (A8) we find

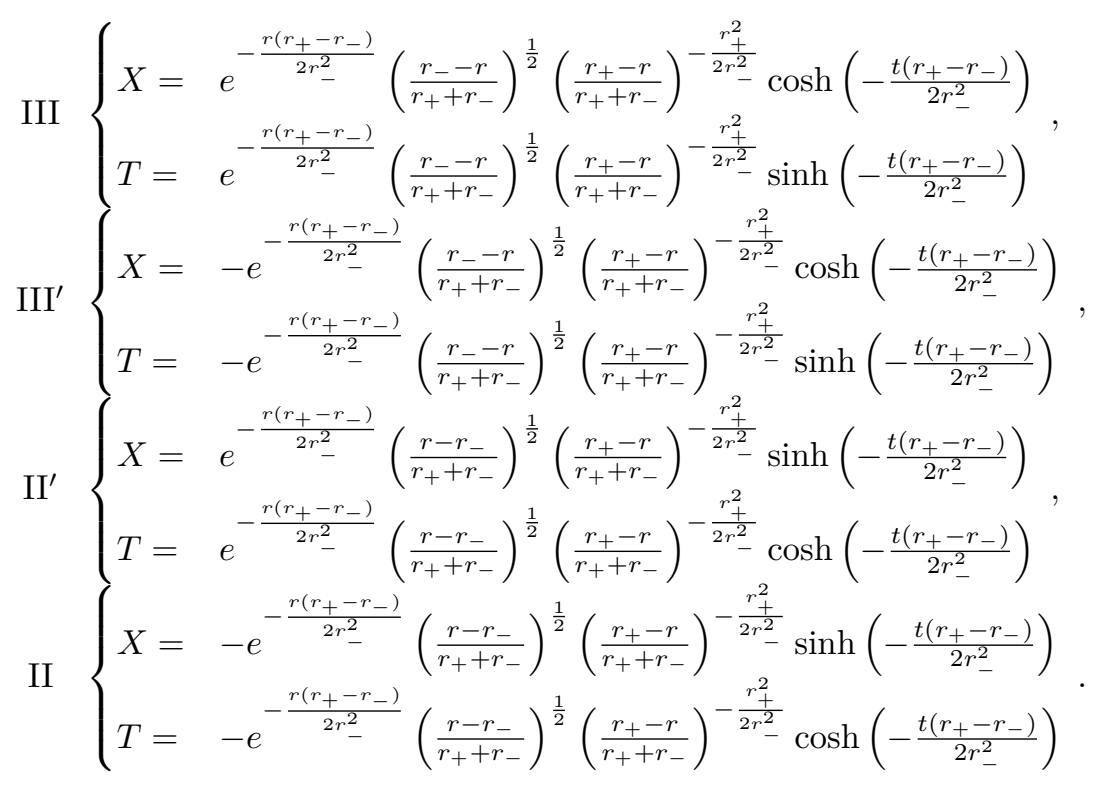

These relations can then be used to find the transformation that gives the coordinate $t$ in terms of the coordinates $X$ and $T$, such that

$$
\begin{array}{ll}
t=-\frac{2 r_{-}^{2}}{r_{+}-r_{-}} \operatorname{arctanh}\left(\frac{T}{X}\right) \quad, \text { in regions III and III', } \\
t=-\frac{2 r_{-}^{2}}{r_{+}-r_{-}} \operatorname{arctanh}\left(\frac{X}{T}\right) \quad, \text { in regions II and } \text { II' }^{\prime}
\end{array}
$$

The spacetime diagram for this coordinate patch together with the relevant coordinate transformations are exhibited graphically in Figure A2. 


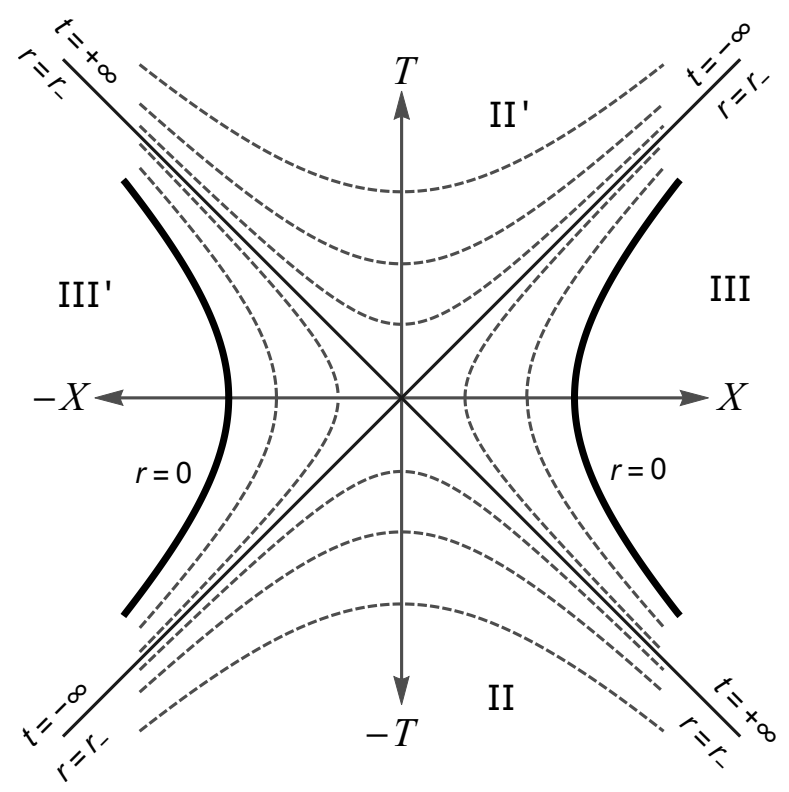

Figure A2: Relation between the Kruskal-Szekeres coordinates $\{X, T\}$ that cover a neighborhood of the Cauchy horizon and the Schwarzschild coordinates $\{r, t\}$. The hyperbolas represent curves of constant $r$ coordinate while curves of constant $t$ are straight lines through the origin. The thick black line represent the singularity at $r=0$.

\section{Appendix B: Extrinsic curvature as seen from $\mathcal{M}_{\mathrm{e}}$ in a nonextremal Reissner-Nordström spacetime}

\section{Boundary surface outside the event horizon}

We want to calculate the extrinsic curvature of a shell $\mathcal{S}$ in a nonextremal Reissner-Nordström spacetime, see also the nomenclature and some details in Section II A. Assuming the matching surface to be timelike, static and spherically symmetric, one finds that the nontrivial components of the extrinsic curvature of the matching hypersurface $\mathcal{S}$ are given by

$$
K_{\tau \tau}=-a^{\alpha} n_{\alpha}, \quad K_{\theta \theta}=\nabla_{\theta} n_{\theta}, \quad K_{\varphi \varphi}=\nabla_{\varphi} n_{\varphi},
$$

where $a^{\alpha} \equiv u^{\beta} \nabla_{\beta} u^{\alpha}$ is the acceleration of an observer with four-velocity $u^{\alpha}$ comoving with $\mathcal{S}, n^{\alpha}$ is the normal to $\mathcal{S}$, and $\nabla_{\alpha}$ is the covariant derivative using the Levi-Civita connection. In our study we will allow the shell to be located at any region of the nonextremal Reissner-Nordström spacetime. It is possible to find a coordinate system that covers the entire Reissner-Nordström spacetime without coordinate singularities. For our analysis it is simpler to define, instead and as was done in Appendix A, two coordinate patches to describe the various regions of the Reissner-Nordström spacetime exterior to the shell. Hence, we will separate the study of a shell located in a region described by one coordinate patch and the other.

Here we will make the derivation of the expressions for the induced metric on $\mathcal{S}$ and the extrinsic curvature components as seen from the exterior spacetime, $\mathcal{M}_{\mathrm{e}}$, in the nonextremal state, $r_{+}>r_{-}$, i.e., $M>Q$, and with $\mathcal{S}$ having radius $R$ obeying $R>r_{+}$, i.e., $\mathcal{S}$ is located outside the sphere defined by the gravitational radius or outside the event horizon, depending on the orientation of $\mathcal{S}$ itself.

We start by studying the properties of a static shell located in a region described by the coordinate patch defined in Appendix A, the coordinate patch without the coordinate singularity at the event horizon $r=r_{+}$. In this region, the line element for the Reissner-Nordström spacetime in Kruskal-Szekeres coordinates is given by, see Section A 2 for details,

$$
\begin{gathered}
d s_{\mathrm{e}}^{2}=4\left(\frac{r_{+}+r_{-}}{r_{+}-r_{-}}\right)^{2} \frac{r_{+}^{4}}{r^{2}} e^{-\frac{r\left(r_{+}-r_{-}\right)}{r_{+}^{2}}}\left(\frac{r-r_{-}}{r_{+}+r_{-}}\right)^{1+\left(\frac{r_{-}}{r_{+}}\right)^{2}}\left(d X^{2}-d T^{2}\right)+r^{2}(X, T) d \Omega^{2}, \\
X^{2}-T^{2}=e^{\frac{r\left(r_{+}-r_{-}\right)}{r_{+}^{2}}}\left(\frac{r-r_{+}}{r_{+}+r_{-}}\right)\left(\frac{r-r_{-}}{r_{+}+r_{-}}\right)^{-\left(\frac{r_{-}}{r_{+}}\right)^{2}} .
\end{gathered}
$$


The components of the 4-velocity of an observer comoving with $\mathcal{S}$ as seen from $\mathcal{M}_{\mathrm{e}}$ are,

$$
u_{\mathrm{e}}^{\alpha}=\sqrt{\frac{g^{X X}}{X^{2}-T^{2}}}(X, T, 0,0)
$$

where the sign was chosen so that $u$ points to the future and $g^{X x}$ is the $X X$ component of the inverse metric associated with Eq. (B2). We use $R$ to describe the radial coordinate of the shell and so, the intrinsic line element of $\mathcal{S}$ is

$$
\left.d s^{2}\right|_{\mathcal{S}}=-d \tau^{2}+R^{2} d \Omega^{2}
$$

Imposing that the normal points in the direction of increasing $X$ coordinate implies that the choice of the sign is different if we consider the shell to be in the region I or $\mathrm{I}^{\prime}$, see Figure 2 and also Figure A1. Then the normal $n_{\mathrm{e} \alpha}$ is

$$
n_{\mathrm{e} \alpha}=\operatorname{sign}(X) \sqrt{\frac{g_{X X}}{X^{2}-T^{2}}}(T,-X, 0,0) .
$$

where the quantities on the right-hand side are to be evaluated at $r=R$ and $\operatorname{sign}(X)$ is the signum function of the coordinate $X$ of the shell. Having found the normal to the hypersurface $\mathcal{S}$ as seen from the exterior nonextremal Reissner-Nordström spacetime, we can now compute the nonzero components of the extrinsic curvature.

Anticipating some of the intermediate results, we first find the derivative of the radial coordinate $r$ in order to the coordinates $\{X, T\}$. Taking the derivative of the second of the equations given in Eq. (B2) in order to $X$ and $T$ independently, we find that $\frac{\partial r}{\partial X}=\frac{g_{X X}}{2} \frac{r_{+}-r_{-}}{r_{+}^{2}} X$ and $\frac{\partial r}{\partial T}=-\frac{g_{X X}}{2} \frac{r_{+}-r_{-}}{r_{+}^{2}} T$, as well as $\frac{\partial r}{\partial T}=-\frac{T}{X} \frac{\partial r}{\partial X}$. The Christoffel symbols are given by $\Gamma_{\alpha \beta}^{\gamma}=\frac{1}{2} g^{\gamma \sigma}\left(g_{\alpha \sigma, \beta}+g_{\beta \sigma, \alpha}-g_{\alpha \beta, \sigma}\right)$. So, to compute the Christoffel symbols associated with the metric (B2) we need to find the derivatives of the metric components. Noting that $g_{T T}=-g_{X X}$, and using the three previous equations we find $\partial_{X} g_{X X}=\frac{\left(g_{X X}\right)^{2}}{r-r_{-}} \frac{r_{+}-r_{-}}{2 r_{+}^{4}}\left[2 \frac{r_{+}^{2} r_{-}}{r}-\left(r_{+}-r_{-}\right)\left(r_{+}+r\right)\right] X, \partial_{X} g_{T T}=-\partial_{X} g_{X X}$, $\partial_{T} g_{X X}=-\frac{T}{X} \partial_{X} g_{X X}, \partial_{T} g_{T T}=\frac{T}{X} \partial_{X} g_{X X}$. One can find the Christoffel symbols needed to compute the component $K_{\tau \tau}$ of the extrinsic curvature. They are $\Gamma_{X X}^{X}=\frac{g_{X X}}{4 r_{+}^{4}} \frac{r_{+}-r_{-}}{r-r_{-}}\left[2 \frac{r_{+}^{2} r_{-}}{r}-\left(r_{+}-r_{-}\right)\left(r_{+}+r\right)\right] X, \Gamma_{T T}^{X}=\Gamma_{X X}^{X}, \Gamma_{X T}^{X}=$ $\Gamma_{T X}^{X}=-\frac{T}{X} \Gamma_{X X}^{X}, \Gamma_{T T}^{T}=-\frac{T}{X} \Gamma_{X X}^{X}, \Gamma_{X X}^{T}=-\frac{T}{X} \Gamma_{X X}^{X}, \Gamma_{T X}^{T}=\Gamma_{X T}^{T}=\Gamma_{X X}^{X}$. Substituting these into the acceleration vector $a^{\alpha}$, we find that components $a^{X}$ and $a^{T}$ of the acceleration vector field are given by $a^{X}=\frac{d U^{X}}{d \tau}+\frac{X^{2}-T^{2}}{T^{2}} \Gamma_{X X}^{X} U^{X} U^{X}$, $a^{T}=\frac{d U^{T}}{d \tau}+\frac{X^{2}-T^{2}}{X T} \Gamma_{X X}^{X} U^{X} U^{X}$, where the repetition of the indices does not mean summation but actual products of the components. Substituting Eq. (B3) in these two equations we find, $a^{X}=\frac{d U^{X}}{d \tau}+g^{X X} \Gamma_{X X}^{X}$ and $a^{T}=\frac{d U^{T}}{d \tau}+g^{X X} \Gamma_{X X}^{X} \frac{T}{X}$. We are now in position to compute the $K_{\mathrm{e} \tau \tau}$ component of the extrinsic curvature of $\mathcal{S}$ given in Eq. (B1) embedded in the exterior nonextremal Reissner-Nordström spacetime. Indeed, with the components $a^{X}$ and $a^{T}$, Eq. (B5), and using $\frac{\partial X}{\partial \tau}=\frac{T}{X} \frac{\partial T}{\partial \tau}$ that we encountered before, in Eq. (B1) yields, $K_{\mathrm{e} \tau \tau}=-\operatorname{sign}(X) \sqrt{\frac{g_{X X}}{X^{2}-T^{2}}}\left(g^{X X}+g^{X X} \Gamma_{X X}^{X} \frac{X^{2}-T^{2}}{X}\right)$. Then, using Eqs. (B2), and $\Gamma_{X X}^{X}$ above, yields $K_{\mathrm{e}}{ }^{\tau} \tau=\frac{\operatorname{sign}(X)}{2 R^{2} k}\left(r_{+}+r_{-}-2 \frac{r_{-} r_{+}}{R}\right)$, where the induced metric in Eq. (B4) was used to raise the indices and $k=\sqrt{\left(1-\frac{r_{+}}{R}\right)\left(1-\frac{r_{-}}{R}\right)}$. To find the other nonzero components of the extrinsic curvature of $\mathcal{S}$ embedded in the exterior nonextremal Reissner-Nordström spacetime, $K_{\mathrm{e} \theta \theta}$ and $K_{\mathrm{e} \varphi \varphi}$, we have to compute the remaining entries of the Christoffel symbols. Equation (B2) and $\frac{\partial r}{\partial T}=-\frac{T}{X} \frac{\partial r}{\partial X}$, yield $\Gamma_{\theta \theta}^{X}=-r g{ }^{X X} \frac{\partial r}{\partial X}$, $\Gamma_{\theta \theta}^{T}=-r g^{X X} \frac{T}{X} \frac{\partial r}{\partial X}, \Gamma_{\varphi \varphi}^{X}=-r \sin ^{2} \theta g^{X X}, \Gamma_{\varphi \varphi}^{T}=-r \sin ^{2} \theta g^{X X} \frac{T}{X} \frac{\partial r}{\partial X}$. Substituting these Christoffel symbols and previous found equations into Eq. (B1) we find $K_{\mathrm{e}}{ }_{\theta}^{\theta}=K_{\mathrm{e}}{ }_{\varphi}=\frac{\operatorname{sign}(X)}{2} \frac{r_{+}-r_{-}}{r_{+}^{2}} \frac{\sqrt{g_{X X}\left(X^{2}-T^{2}\right)}}{R}$. Thus, in brief

$$
K_{\mathrm{e}}{ }^{\tau} \tau=\frac{\operatorname{sign}(X)}{2 R^{2} k}\left(r_{+}+r_{-}-2 \frac{r_{-} r_{+}}{R}\right), \quad K_{\mathrm{e}}{ }_{\theta}{ }_{\theta}=K_{\mathrm{e}}^{\varphi} \varphi=\frac{\operatorname{sign}(X)\left(r_{+}-r_{-}\right)}{2 r_{+}^{2} R} \sqrt{g_{X X}\left(X^{2}-T^{2}\right)},
$$

where $k=\sqrt{\left(1-\frac{r_{+}}{R}\right)\left(1-\frac{r_{-}}{R}\right)}$. With these geometrical quantities one can compute the physical quantities of a thin shell, such as its energy density and tangential pressure, assuming it is made of a perfect fluid, at the boundary surface outside the event horizon, as we did in the text.

\section{Boundary surface inside the Cauchy horizon}

Here we will make the derivation of the expressions for the induced metric on $\mathcal{S}$ and the extrinsic curvature components as seen from the exterior spacetime, $\mathcal{M}_{\mathrm{e}}$, in the nonextremal state, $r_{+}>r_{-}$, i.e., $M>Q$, and with $\mathcal{S}$ 
having radius $R$ obeying $R<r_{-}$, i.e. $\mathcal{S}$ is located inside the sphere defined by the Cauchy radius or inside the Cauchy horizon depending on the orientation of $\mathcal{S}$ itself.

We start by studying the properties of a static shell located in a region described by the coordinate patch defined in Appendix A, the coordinate patch without the coordinate singularity at the Cauchy horizon $r=r_{-}$. In this region, the line element for the Reissner-Nordström spacetime in Kruskal-Szekeres coordinates is given by, see Section A 2 for details,

$$
\begin{gathered}
d s_{\mathrm{e}}^{2}=4\left(\frac{r_{+}+r_{-}}{r_{+}-r_{-}}\right)^{2} \frac{r_{-}^{4}}{r^{2}} \mathrm{e}^{\frac{r\left(r_{+}-r_{-}\right)}{r_{-}^{2}}}\left(\frac{r_{+}-r}{r_{+}+r_{-}}\right)^{1+\left(\frac{r_{+}}{r_{-}}\right)^{2}}\left(d X^{2}-d T^{2}\right)+r^{2}(X, T) d \Omega^{2}, \\
X^{2}-T^{2}=\mathrm{e}^{-\frac{r\left(r_{+}-r_{-}\right)}{r_{-}^{2}}}\left(\frac{r_{-}-r}{r_{+}+r_{-}}\right)\left(\frac{r_{+}-r}{r_{+}+r_{-}}\right)^{-\left(\frac{r_{+}}{r_{-}}\right)^{2}} .
\end{gathered}
$$

The components of the 4 -velocity of an observer comoving with the as seen from $\mathcal{M}_{\mathrm{e}}$ are,

$$
u_{\mathrm{e}}^{\alpha}=-\sqrt{\frac{g^{X X}}{X^{2}-T^{2}}}(X, T, 0,0),
$$

where the sign was chosen so that $u$ points to the future and $g^{X X}$ is the $X X$ component of the inverse metric associated with Eq. (B7). We use $R$ to describe the radial coordinate of the shell and so, the intrinsic line element of $\mathcal{S}$, is

$$
\left.d s^{2}\right|_{\mathcal{S}}=-d \tau^{2}+R^{2} d \Omega^{2} .
$$

Imposing that the normal points in the direction of increasing $X$ coordinate implies that the choice of the sign is different if we consider the shell to be in the region III or III', see Figure 2 and also Figure A2. Then the normal $n_{\mathrm{e} \alpha}$ is

$$
n_{\mathrm{e} \alpha}=\operatorname{sign}(X) \sqrt{\frac{g_{X X}}{X^{2}-T^{2}}}(-T, X, 0,0)
$$

where the quantities on the right-hand side are to be evaluated at $r=R$ and $\operatorname{sign}(X)$ is the signum function of the coordinate $X$ of the shell. Having found the normal to the hypersurface $\mathcal{S}$ as seen from the exterior nonextremal Reissner-Nordström spacetime, we can now compute the nonzero components of the extrinsic curvature.

Let us now compute the nonzero components of the extrinsic curvature of $\mathcal{S}$ for a thin shell inside the Cauchy horizon. Similarly to the previous subsection, we have first to compute some intermediate quantities. Taking the derivative to $X$ and $T$, independently, of the second of the equations given in Eq. (B7) we find $\frac{\partial r}{\partial X}=-\frac{g_{X X}}{2} \frac{r_{+}-r_{-}}{r_{-}^{2}} X$ and $\frac{\partial r}{\partial T}=\frac{g_{X X}}{2} \frac{r_{+}-r_{-}}{r_{-}^{2}} T$. These latter expressions are then related by $\frac{\partial r}{\partial T}=-\frac{T}{X} \frac{\partial r}{\partial X}$. Equation (B7) and $\frac{\partial r}{\partial X}=$ $-\frac{g_{X X}}{2} \frac{r_{+}-r_{-}}{r_{-}^{2}} X$, yield $\partial_{X} g_{X X}=\frac{\left(g_{X X}\right)^{2}}{2 r_{-}^{4}} \frac{r_{+}-r_{-}}{r_{+}-r}\left[2 \frac{r_{-}^{2} r_{+}}{r}+\left(r_{+}-r_{-}\right)\left(r+r_{-}\right)\right] X$. Now, in this coordinate patch one still has $\frac{\partial r}{\partial T}=-\frac{T}{X} \frac{\partial r}{\partial X}$ and $g_{T T}=-g_{X X}$, the other derivatives of the metric are also $\partial_{X} g_{T T}=-\partial_{X} g_{X X}, \partial_{T} g_{X X}=$ $-\frac{T}{X} \partial_{X} g_{X X}, \partial_{T} g_{T T}=\frac{T}{X} \partial_{X} g_{X X}$, where $g_{X X}$ refers here to the $X X$ component of the metric in Eq. (B7). From Eq. (B7) and the one just found for $\partial_{X} g_{X X}$ we find $\Gamma_{X X}^{X}=\frac{g_{X X}}{4 r_{-}^{4}} \frac{r_{+}-r_{-}}{r_{+}-r}\left[2 \frac{r_{-}^{2} r_{+}}{r}+\left(r_{+}-r_{-}\right)\left(r_{+} r_{-}\right)\right] X$. Unsurprisingly, the other entries of the Christoffel symbols are given by $\Gamma_{T T}^{X}=\Gamma_{X X}^{X}, \Gamma_{X T}^{X}=\Gamma_{T X}^{X}=-\frac{T}{X} \Gamma_{X X}^{X}, \Gamma_{T T}^{T}=-\frac{T}{X} \Gamma_{X X}^{X}$, $\Gamma_{X X}^{T}=-\frac{T}{X} \Gamma_{X X}^{X}, \Gamma_{T X}^{T}=\Gamma_{X T}^{T}=\Gamma_{X X}^{X}$, which imply that the $a^{X}$ and $a^{T}$ components of the acceleration are also given by $a^{X}=\frac{d U^{X}}{d \tau}+\frac{X^{2}-T^{2}}{T^{2}} \Gamma_{X X}^{X} U^{X} U^{X}$ and $a^{T}=\frac{d U^{T}}{d \tau}+\frac{X^{2}-T^{2}}{X T} \Gamma_{X X}^{X} U^{X} U^{X}$, where the repetition of the indices does not mean summation but actual products of the components. Then, using Eq. (B8) we find $a^{X}=\frac{d U^{X}}{d \tau}+g^{X X} \Gamma_{X X}^{X}$ and $a^{T}=\frac{d U^{T}}{d \tau}+g^{X X} \Gamma_{X X}^{X} \frac{T}{X}$. Finally, substituting Eq. (B10), and these two latter equations for $a^{X}$ and $a^{T}$, into Eq. (B1) gives $K_{\mathrm{e} \tau \tau}=\operatorname{sign}(X) \sqrt{\frac{g_{X X}}{X^{2}-T^{2}}}\left[g^{X X}+g^{X X} \Gamma_{X X}^{X} \frac{X^{2}-T^{2}}{X}\right]$. Using then Eq. (B7) and the equation for $\Gamma_{X X}^{X}$ found above, leads to $K_{\mathrm{e}}{ }^{\tau} \tau=\frac{\operatorname{sign}(X)}{2 R^{2} k}\left[r_{+}+r_{-}-2 \frac{r_{-} r_{+}}{R}\right]$, where the induced metric on $\mathcal{S}$, Eq. (B9), was used to raise the indices and $k$ is given by $k=\sqrt{\left(1-\frac{r_{+}}{R}\right)\left(1-\frac{r_{-}}{R}\right)}$. All is left now is to find the Christoffel symbols necessary to compute the components $K_{\mathrm{e}}{ }_{\theta}^{\theta}$ and $K_{\mathrm{e}}{ }_{\phi}{ }_{\phi}$ of the extrinsic curvature of $\mathcal{S}$. However, since the angular part of the metric is the same for both coordinate patches, the Christoffel symbols are also given by $\Gamma_{\theta \theta}^{X}=-r g^{X X} \frac{\partial r}{\partial X}$, $\Gamma_{\theta \theta}^{T}=-r g^{X X} \frac{T}{X} \frac{\partial r}{\partial X}, \Gamma_{\varphi \varphi}^{X}=-r \sin ^{2} \theta g^{X X}, \Gamma_{\varphi \varphi}^{T}=-r \sin ^{2} \theta g^{X X} \frac{T}{X} \frac{\partial r}{\partial X}$. These, in conjunction with Eq. (B10), and the 
equations $\frac{\partial r}{\partial X}=-\frac{g_{X X}}{2} \frac{r_{+}-r_{-}}{r_{-}^{2}} X$ and $\frac{\partial r}{\partial T}=\frac{g_{X X}}{2} \frac{r_{+}-r_{-}}{r_{-}^{2}} T$ found above yield $K_{\mathrm{e}}{ }_{\theta}{ }_{\theta}=K_{\mathrm{e}}^{\varphi} \varphi_{\varphi}=\frac{\operatorname{sign}(X)}{2} \frac{r_{+}-r_{-}}{r_{-}^{2}} \frac{\sqrt{g_{X X}\left(X^{2}-T^{2}\right)}}{R}$. Thus, in brief

$$
K_{\mathrm{e}}{ }^{\tau} \tau=\frac{\operatorname{sign}(X)}{2 R^{2} k}\left[r_{+}+r_{-}-2 \frac{r_{-} r_{+}}{R}\right], \quad K_{\mathrm{e}}{ }^{\theta}{ }_{\theta}=K_{\mathrm{e}}^{\varphi} \varphi_{\varphi}=\frac{\operatorname{sign}(X)\left(r_{+}-r_{-}\right)}{2 r_{-}^{2} R} \sqrt{g_{X X}\left(X^{2}-T^{2}\right)} .
$$

where again $k=\sqrt{\left(1-\frac{r_{+}}{R}\right)\left(1-\frac{r_{-}}{R}\right)}$. With these geometrical quantities one can compute the physical quantities of a thin shell, such as its energy density and tangential pressure, assuming it is made of a perfect fluid, at the boundary surface inside the Cauchy horizon, as we did in the text. 
[1] W. Israel, "Singular hypersurfaces and thin shells in general relativity", Il Nouvo Cimento 44, 10 (1966).

[2] A. Papapetrou and A. Hamoui, "Couches simples de matière en relativité générale", Annales de l'Institute Henri Poincaré A 9, 179 (1968).

[3] A. H. Taub, "Space-times with distribution valued curvature tensors", Journal of Mathematical Physics 21, 1423 (1980).

[4] C. Barrabès and W. Israel, "Thin shells in general relativity and cosmology: The lightlike limit", Phys. Rev. D 43, 1129 (1991).

[5] W. Israel, "Gravitational collapse and causality", Phys. Rev. 153, 1388 (1967).

[6] A. B. Evans, "Relativistic dynamics of spherical counter-rotating dust bodies", Gen. Relativ. Gravit. 8, 155 (1977).

[7] A. Papapetrou and A. Hamoui, "Spherically symmetric surface layers in general relativity - correction of an error", Gen. Relativ. Gravit. 10, 253 (1979).

[8] G. 't Hooft and T. Dray, "The effect of spherical shells of matter on the Schwarzschild black hole", Communications in Mathematical Physics 99, 613 (1985).

[9] S. K. Blau, E. I. Guendelman, and A. H. Guth, "Dynamics of false-vacuum bubbles", Phys. Rev. D 35, 1747 (1987).

[10] J. Frauendiener, C. Hoenselaers, and W. Konrad, "A shell around a black hole", Classical Quantum Gravity 7, 585 (1990).

[11] P. R. Brady, J. Louko, and E. Poisson, "Stability of a shell around a black hole", Phys. Rev. D 44, 1891 (1991).

[12] J. Katz and D. Lynden-Bell, "Tension shells and tension stars", Classical Quantum Gravity 8, 2231 (1991).

[13] G. L. Comer and J. Katz, "Some conditions for the existence of tension stars", Mon. Not. R. Astron. Soc. 267, 51 (1994).

[14] M. Visser, Lorentzian Wormholes. From Einstein to Hawking (Springer, New York, 1996).

[15] J. P. S. Lemos, F. S. N. Lobo, Sergio Q. Oliveira, "Morris-Thorne wormholes with a cosmological constant", Phys. Rev. D 68, 064004 (2003); arXiv:gr-qc/0302049.

[16] R. André, J. P. S. Lemos, and G. M. Quinta, "Thermodynamics and entropy of self-gravitating matter shells and black holes in $d$ dimensions", Phys. Rev. D 99, 125013 (2019); arXiv:1905.05239 [hep-th].

[17] S. E. P. Bergliaffa, M. Chiapparini, L. M. Reyes, "Thermodynamical and dynamical equilibrium of a self-gravitating uncharged thin shell", Eur. Phys. J. C 80, 719 (2020); arXiv:2006.06766 [gr-qc].

[18] V. de la Cruz and W. Israel, 'Gravitational bounce", Il Nuovo Cimento A 51, 744 (1967).

[19] K. Kuchař, "Charged shells in general relativity and their gravitational collapse", Czechoslovak J. Phys. B 18, 435 (1968).

[20] J. E. Chase, "Gravitational instability and collapse of charged fluid shells", Il Nuovo Cimento B 67, 136 (1970).

[21] D. G. Boulware, "Naked singularities, thin shells, and the Reissner-Nordström metric", Phys. Rev. D 8, 2363 (1973).

[22] A. V. Vilenkin and P. I. Fomin, "Schwarzschild sphere and classical electron self-energy problem", Il Nuovo Cimento A 45 59 (1978).

[23] W. A. Hiscock, "On the topology of charged spherical collapse", Journal of Mathematical Physics 22, 215 (1981).

[24] J. P. S. Lemos and V. T. Zanchin, "Gravitational magnetic monopoles and Majumdar-Papapetrou stars", J. Math. Phys. 47042504 (2006); arXiv:gr-qc/0603101.

[25] G. A. S. Dias, S. Gao, and J. P. S. Lemos, "Charged shells in Lovelock gravity: Hamiltonian treatment and physical implications", Phys. Rev. D 75, 024030 (2007); arXiv:gr-qc/0612072.

[26] S. Gao and J. P. S. Lemos, "Collapsing and static thin massive charged dust shells in a Reissner-Nordström black hole background in higher dimensions", Int. J. Mod. Phys. A 23, 2943 (2008); arXiv:0804.0295 [hep-th].

[27] H. Andréasson, "Sharp bounds on the critical stability radius for relativistic charged spheres", Communications in Mathematical Physics 288, 715 (2009); arXiv:0804.1882 [gr-qc].

[28] H. Andréasson, M. Eklund and G. Rein, "A numerical investigation of the steady states of the spherically symmetric Einstein-Vlasov-Maxwell system”, Classical Quantum Gravity 26, 145003 (2009); arXiv:0903.4092 [gr-qc].

[29] G. A. S. Dias and J. P. S. Lemos, "Thin-shell wormholes in $d$-dimensional general relativity: Solutions, properties, and stability", Phys. Rev. D 82, 084023 (2010); arXiv:1008.3376 [gr-qc].

[30] J. P. S. Lemos and V. T. Zanchin, "Regular black holes: Electrically charged solutions, Reissner-Nordström outside a de Sitter core", Phys. Rev. D 83124005 (2011); arXiv:1104.4790 [gr-qc].

[31] J. P. S. Lemos and O. B. Zaslavskii, "Quasiblack holes: Properties and Carter-Penrose diagrams", in Proceedings of the 13th Marcel Grossmann Meeting (Stockholm, July 2012), edited by K. Rosquist et al (World Scientific, Singapore 2015), p. 1195.

[32] V. A. Berezin and V. I. Dokuchaev, Global Geometry of Space-Time with the Charged Shell (Nova Science Publishers, New York 2014); arXiv:1404.2726 [gr-qc].

[33] J. P. S. Lemos, G. M. Quinta, and O. B. Zaslavskii, "Entropy of a self-gravitating electrically charged thin shell and the black hole limit", Phys. Rev. D 91, 104027 (2015); arXiv:1503.00018 [hep-th].

[34] J. P. S. Lemos, G. M. Quinta, and O. B. Zaslavskii, "Entropy of an extremal electrically charged thin shell and the extremal black hole", Phys. Lett. B 750, 306 (2015); arXiv:1505.05875 [hep-th].

[35] P. Luz and J. P. S. Lemos, "Electrically charged tension shells", in Proceedings of the Fourteenth Marcel Grossmann Meeting, edited by M. Bianchi et al (World Scientific, Singapore, 2017), p. 1641.

[36] M. Thaller, "Existence of static solutions of the Einstein-Vlasov-Maxwell system and the thin shell limit", SIAM J. Math. Anal. 51, 2231 (2019); arXiv:1801.05664 [gr-qc].

[37] J. P. S. Lemos and O. B. Zaslavskii, "Compact objects in general relativity: From Buchdahl stars to quasiblack holes", Int. J. Mod. Phys. D 29, 2041019 (2020); arXiv:2007.1094 [gr-qc].

[38] J. C. Graves and D. R. Brill, "Oscillatory character of Reissner-Nordström metric for an ideal charged wormhole", Phys. 
Rev. 120, 1507 (1960).

[39] R. Penrose, "Structure of space-time", in Battelle Rencontres: 1967 Lectures in Mathematics and Physics, edited by C. M. DeWitt and J. A. Wheeler (Benjamin, New York 1968), p. 121.

[40] B. Carter, "The complete analytic extension of the Reissner-Nordström metric in the special case $e^{2}=m^{2} "$, Phys. Lett. 21, 423 (1966).

[41] S. W. Hawking and G. F. R. Ellis, The Large Scale Structure of Spacetime (Cambridge University Press, Cambridge 1973).

[42] C. W. Misner, K. S. Thorne, and J. A. Wheeler, Gravitation (W. H. Freeman, San Francisco 1973).

[43] F. de Felice and C. J. S. Clarke, Relativity on Curved Manifolds (Cambridge University Press, Cambridge, 1990). 